Accepted for Publication in The Publications of the Astronomical Society of the Pacific

\title{
The Distance to SN 1999em in NGC 1637 from the Expanding Photosphere Method
}

\author{
Douglas C. Leonard ${ }^{1,2}$, Alexei V. Filippenko ${ }^{1}$, Elinor L. Gates ${ }^{3}$, Weidong Li ${ }^{1}$, Ronald G. \\ Eastman $^{4,5}$, Aaron J. Barth ${ }^{6}$, Schelte J. Bus ${ }^{7}$, Ryan Chornock ${ }^{1}$, Alison L. Coil ${ }^{1}$, Sabine Frink ${ }^{8}$, \\ Carol A. Grady ${ }^{9}$, Alan W. Harris ${ }^{10}$, Matthew A. Malkan ${ }^{11}$, Thomas Matheson ${ }^{1,12}$, Andreas \\ Quirrenbach $^{9}$, and Richard R. Treffers ${ }^{1}$
}

\begin{abstract}
We present 30 optical spectra and 49 photometric epochs sampling the first 517 days after discovery of supernova (SN) $1999 \mathrm{em}$, and derive its distance through the expanding photosphere method (EPM). SN 1999em is shown to be a Type II-plateau (II-P) event, with a photometric plateau lasting until about 100 days after explosion. We identify the dominant ions responsible for most of the absorption features seen in the optical portion of the spectrum during the plateau phase. Using the weakest unblended absorption features to estimate photospheric velocity, we find the distance to SN 1999em to be $D=8.2_{-0.6}^{+0.6} \mathrm{Mpc}$, with an explosion date of HJD 2, 451,475.6 \pm 1.4 , or $5.3 \pm 1.4$ days before discovery. We show that this distance estimate is about $10 \%$ closer than the distance that results if the strong Fe II $\lambda \lambda 4924$, 5018, 5169 absorption features,
\end{abstract}

\footnotetext{
${ }^{1}$ Department of Astronomy, University of California, Berkeley, California 94720-3411

${ }^{2}$ Present address: Department of Astronomy, University of Massachusetts, Amherst, MA 01003-9305; leonard@nova.astro.umass.edu

${ }^{3}$ Lick Observatory, PO Box 82, Mount Hamilton, CA 95140

${ }^{4}$ Lawrence Livermore National Laboratory, Livermore, CA 94551

${ }^{5}$ Department of Astronomy and Astrophysics, University of California, Santa Cruz, CA 95064

${ }^{6}$ Harvard-Smithsonian Center for Astrophysics, 60 Garden St., MS-20, Cambridge, MA 02138

${ }^{7}$ University of Hawaii, Institute for Astronomy, 640 N. A'ohoku Place \#209, Hilo, HI 96720

${ }^{8}$ Department of Physics, University of California, San Diego, Center for Astrophysics and Space Sciences, 9500 Gilman Drive, La Jolla, CA 92093-0424

${ }^{9}$ NASA Goddard Space Flight Center, Code 685, Greenbelt, MD 20771

${ }^{10}$ Jet Propulsion Laboratory, MS 183-501, 4800 Oak Grove Dr., Pasadena, CA 91109-8099

${ }^{11}$ Department of Physics and Astronomy, University of California, Los Angeles, CA 90095-1562

${ }^{12}$ Present address: Harvard-Smithsonian Center for Astrophysics, 60 Garden St., MS-20, Cambridge, MA 02138
} 
which have often been used in previous EPM studies, are used to estimate photospheric velocity.

We examine potential sources of systematic error in EPM-derived distances, and find the most significant to result from uncertainty in the theoretical modeling of the flux distribution emitted by the SN photosphere (i.e., the "flux dilution factor"). We compare previously derived EPM distances to 8 SNe II in galaxies (or members of the same group) for which a recently revised Cepheid distance exists from the HST Key Project and find $D_{\text {Cepheids }} / D_{\mathrm{EPM}}=0.87 \pm 0.06$ (statistical); eliminating the $3 \mathrm{SNe}$ II distances for which a Cepheid distance only exists to a group member, and not the host galaxy itself, yields $D_{\text {Cepheids }} / D_{\mathrm{EPM}}=0.96 \pm 0.09$. Additional direct comparisons, especially to spectroscopically and photometrically normal SNe II-P, will certainly help to produce a more robust comparison.

Finally, we investigate the possible use of SNe II-P as standard candles and find that for 8 photometrically confirmed SNe II-P with previously derived EPM distances and SN $1999 \mathrm{em}$, the mean plateau absolute brightness is $\bar{M}_{V}$ (plateau) $=-16.4 \pm 0.6$ mag, implying that distances good to $\sim 30 \%(1 \sigma)$ may be possible without the need for a complete EPM analysis. At $\bar{M}_{V}$ (plateau) $=-15.9 \pm 0.2 \mathrm{mag}$, SN $1999 \mathrm{em}$ is somewhat fainter than the average SN II-P. The general consistency of absolute SNe II-P brightness during the plateau suggests that the standard candle assumption may allow SNe II-P to be viable cosmological beacons at $z>2$.

Subject headings: cosmology: observations - distance scale - galaxies: individual (NGC 1637) — supernovae: individual (SN 1999em)

\section{INTRODUCTION}

"Normal" core-collapse supernovae ( $\mathrm{SNe}$ ) are thought to result from isolated, massive stars (initial mass $z 8-10 M_{\odot}$ ) with thick hydrogen envelopes (generally several solar masses) intact at the time of the explosion. Their light curves show a distinct plateau (hence the moniker "SN II-P"), resulting from an enduring period (sometimes as long as 150 days) of nearly constant luminosity as the hydrogen recombination wave recedes through the envelope and slowly releases the energy deposited by the shock and by radioactive decay. It is to these events that the expanding photosphere method (EPM) of extragalactic distance determination is most accurately applied (e.g., Eastman, Schmidt, \& Kirshner 1996, hereafter E96).

SNe II have not traditionally been treated as standard candles. Through calibration with the EPM, however, they have provided direct measurements of distances independent of all the uncertain rungs of the extragalactic distance ladder. While EPM distances have been derived 
to both SNe II-P and SNe II-L, ${ }^{13}$ it is thought to be most securely applied to SNe II-P since the theoretical modeling of their flux distribution is much simpler and better understood. Thus far, the EPM has been applied to 18 SNe II, including 10 SNe II-P, spanning distances from $0.049 \mathrm{Mpc}$ (SN 1987A) to $180 \mathrm{Mpc}$ (SN 1992am; Schmidt et al. 1994b). Using SNe II alone, a Cepheid-independent value of $H_{\circ}=73 \pm 7 \mathrm{~km} \mathrm{~s}^{-1} \mathrm{Mpc}^{-1}$ has been derived (Schmidt et al. 1994a). Despite EPM's apparent success, however, there is considerable debate concerning possible sources of systematic error. In this paper, we derive an EPM distance to the most thoroughly observed SN II-P to date, SN $1999 \mathrm{em}$, and critically evaluate possible sources of systematic error in the EPM technique.

EPM is fundamentally a geometric technique, a variant of the Baade (1926) method used to determine the distance to variable stars: the linear radius of the expanding photosphere, $R$, is compared with the photosphere's angular size, $\theta$, to derive the distance to the SN, $D$. Naturally, since all extragalactic SNe are unresolved during the "photospheric" phase (the plateau in a SN II$\mathrm{P}), R$ and $\theta$ must be derived rather than measured directly.

In the original formulation of EPM (Kirshner \& Kwan 1974), the theoretical angular size of the $\mathrm{SN}$ photosphere is approximated as

$$
\theta=\frac{R}{D}
$$

where $R$ the photosphere's radius and $D$ the distance to the SN. If spherical geometry is assumed and the ejecta are in free expansion (i.e., deceleration due to gravity or swept-up interstellar material is negligible, and the radius of the progenitor is small compared with the size of the photosphere) equation (1) may be written as

$$
\theta=\frac{v_{\text {phot }}\left(t-t_{\circ}\right)}{D}
$$

where $v_{\text {phot }}$ is the velocity of material instantaneously at the photosphere at time $t$, and the explosion occurred at time $t_{0}$. Rewriting this equation in the suggestive form

$$
t=D\left(\frac{\theta}{v_{\text {phot }}}\right)+t_{\circ}
$$

we see that plotted measurements of at least two epochs of $\theta / v_{\text {phot }}$ against $t$ should result in a line with slope $D$ and $y$-intercept $t_{\circ}$. A particularly powerful feature of this method is that if the date of explosion $\left(t_{\circ}\right)$ is known (either from pre-discovery images or derived from the EPM technique itself if more than one epoch of data is available), then each measurement of $\theta / v_{\text {phot }}$ provides an independent estimate for $D$. To determine the distance to a $\mathrm{SN}$, then, requires measurements of the theoretical angular size, $\theta$, and the photospheric velocity, $v_{\text {phot }}$.

\footnotetext{
13 "L" for their linearly declining light curves, lacking a plateau; these events are generally believed to result from progenitors that have lost a substantial fraction of their hydrogen envelope prior to exploding (e.g., Filippenko 1997).
} 
Assuming a spherically symmetric SN photosphere that radiates isotropically as a blackbody, conservation of flux demands (temporarily neglecting extinction)

$$
4 \pi R^{2} \pi B_{\nu}\left(T_{c}\right)=4 \pi D^{2} f_{\nu}
$$

where $B_{\nu}\left(T_{c}\right)$ is the Planck function at color temperature $T_{c}$, and $f_{\nu}$ is the flux received at Earth. Solving for $\theta$, this becomes

$$
\theta=\sqrt{\frac{R^{2}}{D^{2}}}=\sqrt{\frac{f_{\nu}}{\pi B_{\nu}\left(T_{c}\right)}}
$$

The observables thus required to derive an EPM distance are $v_{\text {phot }}, f_{\nu}$, and $T_{c}$. Traditionally, $v_{\text {phot }}$ is estimated by measuring the blueshift of the P-Cygni absorption troughs of weak lines seen in the optical spectrum, typically Fe II $\lambda \lambda 4924,5018,5169$ and occasionally Sc II $\lambda 5527$ and Sc II $\lambda 5658$ as well. The implicit assumption is that these lines are optically thin above the photosphere and that the density of the absorbing material is a steeply declining function of radius, with the densest material (which causes the greatest amount of absorption) being instantaneously at the location of the photosphere. Since strong, optically thick lines may form absorption minima at higher velocities (i.e., well above the photosphere), velocities derived from the weakest features are the best indicators of photospheric velocity. In principle, $f_{\nu}$ and $T_{c}$ could be determined with accurate spectrophotometry. In practice, however, spectrophotometry is generally not available, so broadband photometry (usually some subset of $B V I J H K$ ) is used. From several measurement epochs of $v_{\text {phot }}, T_{c}$, and $f_{\nu}$ during the photospheric phase, then, the distance and time of explosion of the $\mathrm{SN}$ are derived.

The current debate over possible sources of systematic error in the EPM technique generally focuses on three main assumptions:

1. The photosphere radiates as a blackbody. On applying the Baade (1926) technique to measure the distance to variable stars, Walter Baade himself wrote: “...the only assumption we had to make [in formulating the technique] was that Cepheids radiate as a blackbody. Further experience has to show how far we can hold this assumption." ${ }^{14}$ It was not until decades later that Wagoner (1982) first demonstrated that a Type II SN photosphere in fact radiates as a "dilute" blackbody due to the dominance of electron scattering over absorption processes. That is, the continuum spectrum that is ultimately released from the electron-scattering photosphere (defined as the surface of last scattering, $\tau_{e}=2 / 3$ ) is produced in the deeper layer at which the radiation field thermalizes to the local gas temperature, known as the "thermalization depth" (see, e.g., E96). This smaller radius is the location of the last true absorption and reemission of the photons that make up the thermal continuum. The blackbody spectrum ultimately released by the electron-scattering photosphere thus possesses a luminosity

\footnotetext{
${ }^{14}$ Passage translated from the original German by M. Modjaz.
} 
appropriate for a smaller radiating surface. The amount the flux is "diluted" is parameterized by the relation

$$
\zeta=\frac{R_{\text {therm }}}{R_{\text {phot }}}
$$

where $R_{\text {therm }}$ is the radius at the thermalization depth, $R_{\text {phot }}$ is the photosphere's radius (i.e., the surface of last scattering), and $\zeta$ is called the "distance correction factor," since its inclusion in equation (4) "corrects" derived distances such that

$$
D_{\text {actual }}=\zeta D_{\text {measured }}
$$

That is, distances derived without taking into account flux dilution will overestimate the distance by a factor $\zeta$. In principle, $\zeta$ could depend on many things, including the chemical composition (i.e., the metallicity) and density structure of the progenitor star, and the expansion rate and luminosity of the SN explosion. Studies of theoretical models of realistic SN atmospheres with a wide range of properties, however, have demonstrated that $\zeta$ is in fact a nearly one-dimensional function of color temperature, $T_{c}$, with only a small density dependence at short wavelengths (E96; Montes \& Wagoner 1995).

From a study of 63 model atmospheres, E96 provide convenient analytic approximations for $\zeta$ as a function of color temperature for a SN II-P, determined for 4 broadband filter combinations, $B V, B V I, V I$, and $J H K$ (the $R$ band is generally not used, since it is dominated by the $\mathrm{H} \alpha$ line, which can vary significantly among SNe II-P). In the models of E96, $\zeta$ is shown to vary smoothly as a function of $T_{c}$, with a $1 \sigma$ scatter among the models of $0.11 \%, 0.05 \%, 0.08 \%$, and $0.07 \%$ for the $B V, B V I, V I$, and $J H K$ filter combinations, respectively. Recently, by taking into account the effects that telluric features have on observed photometric data, as well as a subtle correction to the BVRI filter functions of Bessell [1990] to make them appropriate for use with photon, and not energy, distributions, Hamuy et al. (2001) has provided improved estimates of $\zeta\left(T_{c}\right)$, which we adopt in this paper. ${ }^{15}$ With $B V I$ photometry of SN $1999 \mathrm{em}$, we shall be able to test the self-consistency of the flux-dilution factors by comparing the distances derived using the three filter combinations $B V, B V I$, and $V I$ individually.

The dilution factors of E96 have been used to determine distances to 18 SNe II (Schmidt, Kirshner, \& Eastman 1992; Schmidt et al. 1994a, b; see also Clocchiatti et al. 1995). A first-order check on the precision of the predicted shape of the function relating $\zeta$ and $T_{c}$ is whether multiple observations at different epochs, and therefore different $T_{c}$ and $\zeta$, are consistent. As discussed by Leonard et al. (2001, hereafter L01), previous EPM studies of

\footnotetext{
${ }^{15}$ Hamuy et al. (2001) also derive an EPM distance to SN 1999em. We note that other than adopting the improved dilution factors our analysis here is completely independent of this work, which was posted on astro-ph after this paper was submitted; we do, however, make a brief comparison of the two derived distances, and comment on differences in the techniques used, in $\S 4.1$.
} 
SNe that had a broad temporal range of observational epochs have yielded consistent distances (i.e., a relatively straight line when $\theta / v$ is plotted against $t$ [Equation (3)]). Empirical evidence therefore suggests that the shape of the theoretically derived function relating $\zeta$ and $T_{c}$ must be nearly correct. However, differences of opinion remain concerning the overall level of the dilution factor, with discrepancies in $\zeta$ as high as $60 \%$ claimed by some groups (e.g., Baron et al. 1995). In all, the uncertainty in the dilution factor remains the main point of contention when applying EPM to derive cosmological parameters.

2. The photosphere is spherically symmetric. Recent polarization studies of young core-collapse $\mathrm{SNe}$ (see Wheeler [2000] for a comprehensive list of polarimetric observations of SNe; in general, higher polarization implies greater departure from a sphere), the possible association of SNe with some gamma-ray bursts (e.g., Bloom et al. 1999), and the high velocity of pulsars (Cordes \& Chernoff 1998), all implicate a fundamentally asymmetric explosion for core-collapse SNe. From the polarization studies an interesting trend has also emerged: the degree of asphericity at early times appears to be inversely correlated with the amount of envelope material intact at the time of the explosion. That is, SNe that have lost a significant amount of their hydrogen envelope prior to exploding are found to be more highly polarized at early times than those that have hydrogen envelopes substantially intact (Wheeler 2000; see also Wang et al. 2001). The addition of envelope material evidently serves to dampen the asymmetry at early times, in agreement with the theoretical expectation that asymmetric explosions should turn spherical as they expand through the envelope (e.g., Chevalier \& Soker 1989; Höflich, Wheeler, \& Wang 1999).

The most thorough investigation of the sphericity assumption for a SN II-P is that by L01 for SN 1999em itself. From multiple spectropolarimetric epochs spanning the first 163 days after discovery, L01 find a very low, but perhaps slowly increasing polarization for SN 1999em. Spectropolarimetric observations of other SNe II-P also show weak evidence for intrinsic polarization, suggesting that departures from spherical symmetry may not be substantial at early times for this class of objects (Leonard \& Filippenko 2001). Although the translation from observed polarization to degree of asphericity is obfuscated by uncertainties in the interstellar polarization and SN viewing orientation, when the polarization of SN 1999em is interpreted in terms of the oblate, electron-scattering models of Höflich (1991), a lower bound on the asphericity of $7 \%$ during the middle of the plateau results. If the asphericity implied by the spectropolarimetry results in directionally-dependent flux, it would clearly lead to distance errors in the EPM analysis for SN 1999em (see, e.g., Wagoner, 1991). We caution, however, that although global asphericity is the preferred interpretation of the observed polarization, there are other mechanisms that can produce SN polarization, including scattering by dust (e.g., Wang \& Wheeler 1996) and asymmetrically distributed radioactive material within the SN envelope (e.g., Höflich 1995; Höflich, Khokhlov, \& Wang 2001). Thus, while the question is far from settled, available polarimetric evidence is consistent with small asphericity during the period of interest for EPM. 
Further support for early-time SN sphericity comes from the apparent success of the EPM technique itself. By quantifying the amount of systematic scatter in the EPM Hubble diagram, L01 find that the agreement between previous EPM distance measurements of SNe II and the distances to the host galaxies predicted by a linear Hubble law restrict mean SN II asphericity to values less than $30 \%(3 \sigma)$ during the photospheric epoch. From the available evidence, then, there is growing support for the claim that asphericity does not significantly hamper the cosmological use of SNe II whose progenitors have massive hydrogen envelopes intact at the time of explosion.

3. The photospheric velocity is accurately determined by measuring the blueshift of weak P-Cygni absorption lines. In all previous EPM studies, photospheric velocity has been estimated by measuring the blueshift of the flux minima in the P-Cygni absorption profiles of lines in the optical spectrum (e.g., Kirshner \& Kwan 1974). The main assumptions here are that the lines in the SN envelope are optically thin above the photosphere, and that density is a steeply declining function of radius. We also note that due to the multiple scatters that occur to photons as they travel from the location of the thermalization depth to the surface of last scattering (i.e., $\tau_{e}=2 / 3$, the optical photosphere), line features are not expected to be formed significantly below the optical photosphere (i.e., Hatano et al. 1999; cf., Hamuy et al. [2001]). With these ideas in mind, then, it is clear that the greatest amount of absorption will occur just above the photosphere, and that velocities measured with weak lines should be good photospheric velocity indicators.

The very early-time (less than 10 days after core collapse) spectra of SN 1987A were modeled in detail by Schmutz et al. (1990) and Eastman \& Kirshner (1989). The hydrogen Balmer lines are shown by Eastman \& Kirshner (1989) to yield velocities that become a progressively poorer representation of the photospheric velocity with time: they are within $10 \%$ of the photospheric velocity two days after collapse, but by ten days $\mathrm{H} \alpha, \mathrm{H} \beta$, and $\mathrm{H} \gamma$ yield velocities 1.9, 1.5, and 1.4 times the photospheric velocity, respectively. The explanation for this behavior comes from examining the density structure of the material overlying the photosphere. At early times, the atmosphere is highly ionized and the photosphere is situated in a region with a density that is a very steeply declining function of radius (e.g., Matzner \& McKee 1999). As the atmosphere cools and starts to recombine the lines form in a much more extended region characterized by a flatter density gradient (Eastman \& Kirshner 1989). The flatter the density gradient the more extended the line-forming region becomes, which produces the larger blueshift of the optically thick lines. At very early times, then, we expect all lines to converge on the photospheric velocity, but quite soon thereafter weaker features must be used. Both Schmutz et al. (1990) and Eastman \& Kirshner (1989) conclude that the weak Fe II $\lambda \lambda 4924,5018,5169$ lines provide reliable photospheric velocities during the early period studied for SN 1987A. Encouraged by these results, these features have been used as photospheric velocity indicators in most subsequent EPM distance determinations (e.g., Schmidt et al. 1992; Schmidt et al. 1994a, b; Clocchiatti et al. 1995), with the occasional use of Sc II $\lambda 5527$ and Sc II $\lambda 5658$ as well. Most of these EPM studies, however, used data 
from significantly later evolutionary epochs than those used for SN 1987A.

It is possible that the Fe II $\lambda \lambda 4924,5018,5169$ features may themselves become optically thick above the photosphere as the temperature cools to the recombination temperature of hydrogen. Hatano et al. (1999) derive the expected local thermodynamic equilibrium (LTE) optical depths at the photosphere as a function of temperature for prominent absorption lines from each of the major ions thought to be responsible for features in a SN atmosphere with solar metallicity. The predicted depths nicely reflect the order of appearance of lines generally seen in SNe II-P as they cool: first hydrogen Balmer (which is shown to become optically thick below 12,000 K) and He I $\lambda 5876$ followed by Fe II, Si II, Sc II, and then other weaker metal lines. Using results derived in $\S 2$ and $\S 3$, Figure 1 illustrates the color-temperature (in this case, the blackbody temperatures determined by the $B V I$ photometry, $T_{\mathrm{BVI}}$ ) evolution of line strengths for SN $1999 \mathrm{em}$. The line strengths and order of appearance are in good qualitative agreement with the predictions of Hatano et al. (1999); of particular interest, Hatano et al. (1999) predict Fe II $\lambda 5018$ to be optically thick above the photosphere at temperatures below $T_{\text {eff }} \approx 7500 \mathrm{~K}$, suggesting that velocities derived using this line during the recombination phase may slightly overestimate photospheric velocity. By comparing the velocity derived from the Fe II $\lambda \lambda 4924,5018,5169$ features with that inferred from weaker lines, we shall empirically test this for SN $1999 \mathrm{em}$.

With the preceding discussion in mind, we now explicitly include the effects of extinction $\left(A_{\nu}\right)$ and flux dilution $(\zeta)$ in equation (4) and rewrite equation (5) as

$$
f_{\nu}=\zeta_{\nu}^{2} \theta^{2} \pi B_{\nu}\left(T_{c}\right) 10^{-0.4 A_{\nu}}
$$

If accurate spectrophotometry were available, we could solve for $\theta$ and $T_{c}$ by minimizing the quantity $\left(f_{\nu}-\zeta_{\nu}^{2} \theta^{2} \pi B_{\nu}\left[T_{c}\right] 10^{-0.4 A_{\nu}}\right)$ over the observed frequency range. Since such data are seldom obtained we recast equation (8) in terms of broadband photometry. Following E96, we replace $f_{\nu}$ with the observed broadband magnitude (for brevity writing only $B V I$ here) to arrive at

$$
m_{B V I}=-5 \log \theta-5 \log \left[\zeta\left(T_{c}\right)\right]+b_{B V I}+A_{B V I},
$$

where $b_{B V I}$ is the broadband magnitude of the Planck function integrated over the transmission function for the $B, V$, or $I$ bandpass (plus an arbitrary constant). Armed with an estimate of the extinction and a set of photometric magnitudes, we solve for $\theta$ and $T_{c}$ by minimizing the quantity

$$
\varepsilon=\sum_{B V I}\left[m_{B V I}-A_{B V I}+5 \log \theta+5 \log \left[\zeta\left(T_{c}\right)\right]-b_{B V I}\left(T_{c}\right)\right]^{2}
$$

With $v_{\text {phot }}$ measured from spectral lines and $\theta$ derived from photometry, the distance and explosion date of the SN are then determined through equation (3) by finding the best-fitting line to describe 


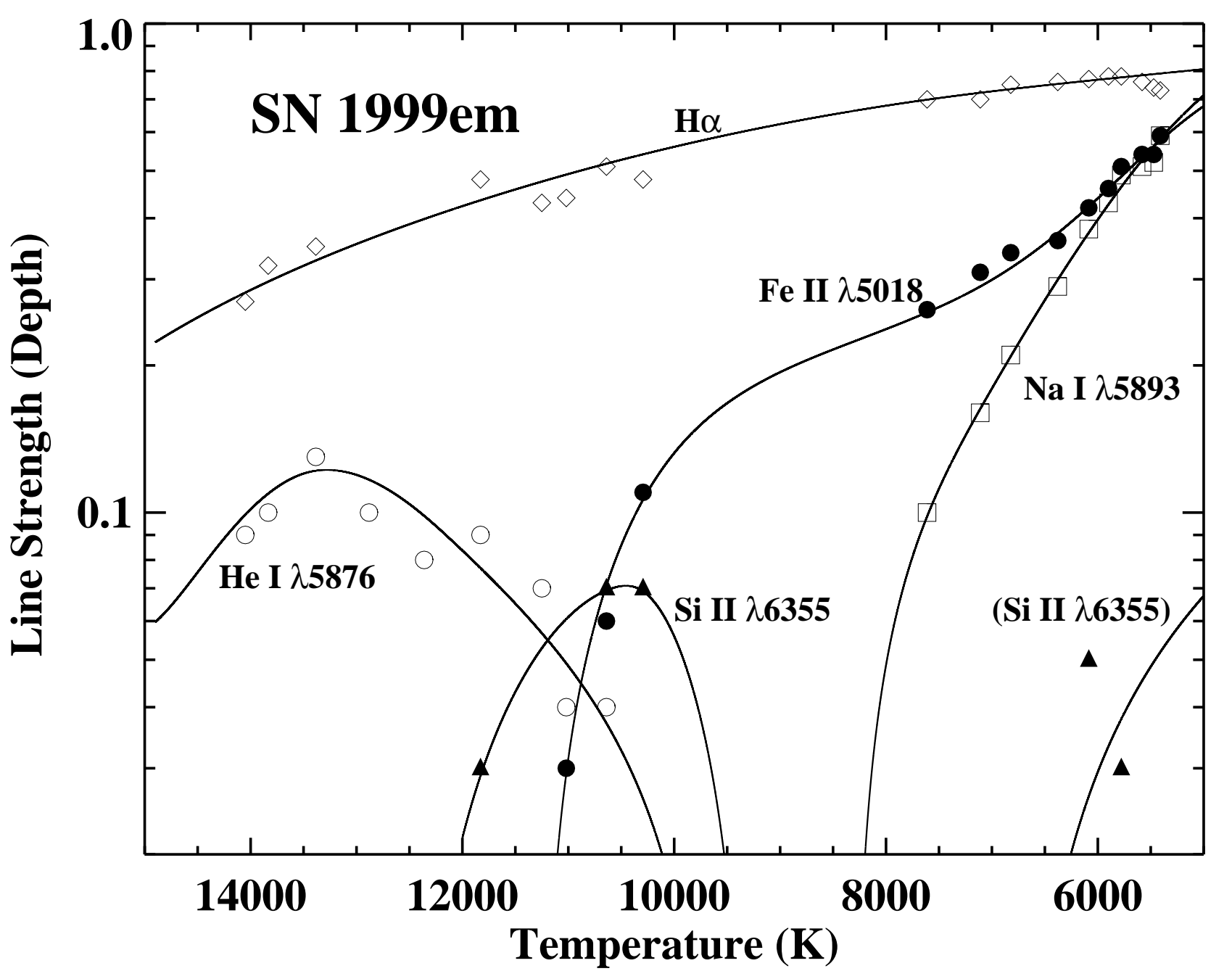

Fig. 1. - The strength of several optical absorption lines plotted against BVI color temperature for SN 1999em. Approximate spline fits to the observations are shown for clarity. Line strength is set equal to the measured line depth, defined as $\left(f_{c}-f_{\min }\right) / f_{c}$, where $f_{\min }$ is the flux at the line's minimum and $f_{c}$ is the value of the flux in the surrounding regions interpolated over the absorption line $(\S 2.2 .3)$. The order of appearance and relative strengths of the lines are in good qualitative agreement with the theoretical predictions of Hatano et al. (1999). Note the presence of Si II $\lambda 6355$ absorption at high photospheric temperature $\left(T_{\mathrm{BVI}}>10,000 \mathrm{~K}\right)$ and then again when the photosphere has cooled to below $T_{\mathrm{BVI}} \approx 6,000 \mathrm{~K}$. 
the data, either through the method of least squares or a more robust (i.e., less sensitive to outliers) estimator, such as the criterion of least absolute deviations (e.g., Press et al. 1992).

SN 1999 em was discovered on 1999 October 29.44 UT (UT dates are used throughout this paper) by $\mathrm{Li}(1999)$ at an unfiltered magnitude of $m \approx 13.5$ mag in the nearly face-on $\left(i \approx 32^{\circ}\right.$, from LEDA $^{16}$ ) SBc galaxy NGC 1637 (Fig. 2). Since an image of the same field on Oct. 20.45 showed nothing at the position of SN 1999em (limiting mag about 19.0; Li 1999), it was likely discovered shortly after explosion. It was quickly identified as a Type II event, with prominent P-Cygni features of hydrogen Balmer and He I $\lambda 5876$ and a blue continuum (Jha et al. 1999). Immediately after discovery a spectral, spectropolarimetric, and photometric campaign was initiated at Lick Observatory. Nearly daily optical photometry and spectroscopy of the young SN were obtained with the $0.8 \mathrm{~m}$ Katzman Automatic Imaging Telescope (KAIT; Treffers et al. 1997; Li et al. 2000; A. V. Filippenko et al., in preparation) and the Lick 1-m Nickel reflector, respectively, providing unprecedented temporal coverage of this Type II event during the photospheric phase. The spectropolarimetric observations are discussed in detail by L01; here we focus on the spectral and photometric data.

In this paper we derive an EPM distance to SN 1999em, and present 30 optical spectra and 49 photometric epochs spanning the first 517 days of its development. We present the photometric data in $\S 2.1$, and the total flux spectra in $\S 2.2$, where we also identify and discuss the dominant ions responsible for the absorption features seen in the optical spectrum during the plateau phase. We consider the reddening of SN $1999 \mathrm{em}$ in $\S 2.3$. We derive and compare the photospheric velocities inferred from the P Cygni absorption minima of several line features in $\S 2.4$, and apply the EPM to SN $1999 \mathrm{em}$ in $\S 3$. We discuss the results in $\S 4$, and summarize our main conclusions in $\S 5$.

\section{Reductions and Analysis}

\subsection{Photometry}

All photometric data were collected using the KAIT, which is equipped with a $512 \times 512$ pixel Apogee CCD camera (AP7) located at the $f / 8.17$ Cassegrain focus, providing a field of view of $6.7 \times 6.7$ with 0. .' 8 per pixel. The "seeing", estimated by the full width at half maximum (FWHM) of stars on the CCD frame, generally ranged from $3^{\prime \prime}$ to $4 . .5$, and exposure times of 3 to 4 minutes in $V R I$ and 5 to 7 minutes in $U B$ were typical during the first 95 days after discovery while the SN was bright and on the plateau portion of its light curve. After SN 1999em dropped off the plateau, exposure times were increased to about 10 minutes.

CCD frames were flatfielded using twilight-sky flats in the usual manner, and cosmic rays were removed interactively by visual inspection of each frame. A noticeable low-level pattern was

\footnotetext{
${ }^{16}$ http://www-obs.univ-lyon1.fr/leda/home_leda.html.
} 
removed from each image by subtracting a dark frame scaled to the same exposure time; however, on some images, particularly the longer $U$ and $B$ exposures, a pattern still remained, most likely caused by temperature fluctuations of the thermoelectrically cooled CCD camera (Modjaz et al. 2001). This was removed by interactively adding or subtracting an additional fraction (generally $<10 \%$ ) of the dark frame, until the pattern disappeared. Photometric tests performed on frames before and after the additional dark subtraction indicate that the low-level pattern has a negligible effect on the photometry. Considerable fringing remained in $I$-band images that did not properly flatten, likely produced by the varying intensity of night-sky emission lines. This also has minimal impact on the photometry since both the SN and the comparison stars were much brighter than the background.

Figure 2 shows a KAIT $B$-band image of NGC 1637 taken on 1999 November 1 . Of the 8 "local standards" identified in the field of SN $1999 \mathrm{em}$, the best were judged to be the three bright, isolated stars labeled $A, B$, and $C$, and they were used to measure the relative $\mathrm{SN}$ brightness on non-photometric nights. The absolute calibration of the field was accomplished on the two photometric nights of 2000 April 3 and September 25 by observing several fields of Landolt (1992) standards over a range of airmasses. The mean UBVRI magnitudes of the stars on the two nights are given in Table 1. The transformation coefficients to the standard Johnson-Cousins (Johnson et al. 1966 for $U B V$; Cousins 1981 for $R I$ ) systems were derived using the solutions from these nights and two additional photometric nights, 2000 September 5 and 27. We determined the instrumental magnitudes for the standards using aperture photometry with the IRAF ${ }^{17}$ DAOPHOT package (Stetson 1987, 1991), which yielded color terms for the KAIT observations of the form

$$
\begin{aligned}
U & =u+0.074(U-B)+C_{U}, \\
B & =b+0.06(B-V)+C_{B}, \\
V & =v-0.04(B-V)+C_{V}, \\
R & =r-0.08(V-R)+C_{R}, \\
I & =i+0.01(V-I)+C_{I},
\end{aligned}
$$

where ubvri are the instrumental and $U B V R I$ the standard Johnson-Cousins magnitudes. The terms $C_{U}, C_{B}, C_{V}, C_{R}$, and $C_{I}$ are the differences between the zero-points of the instrumental and standard magnitudes, determined for each observation by measuring the offset between the instrumental and standard magnitudes and colors of the local standard stars.

The location of SN 1999em at about 23" from the center of NGC 1637 (Figure 2) on the inner edge of a spiral arm gives it a rather complex background. In such situations, it is desirable to wait until the SN fades beyond detection to obtain a "template" observation of the host galaxy, which can then be subtracted from the earlier observations (Filippenko et al. 1986; Hamuy et al. 1994;

\footnotetext{
${ }^{17}$ IRAF is distributed by the National Optical Astronomy Observatories, which are operated by the Association of Universities for Research in Astronomy, Inc., under cooperative agreement with the National Science Foundation.
} 


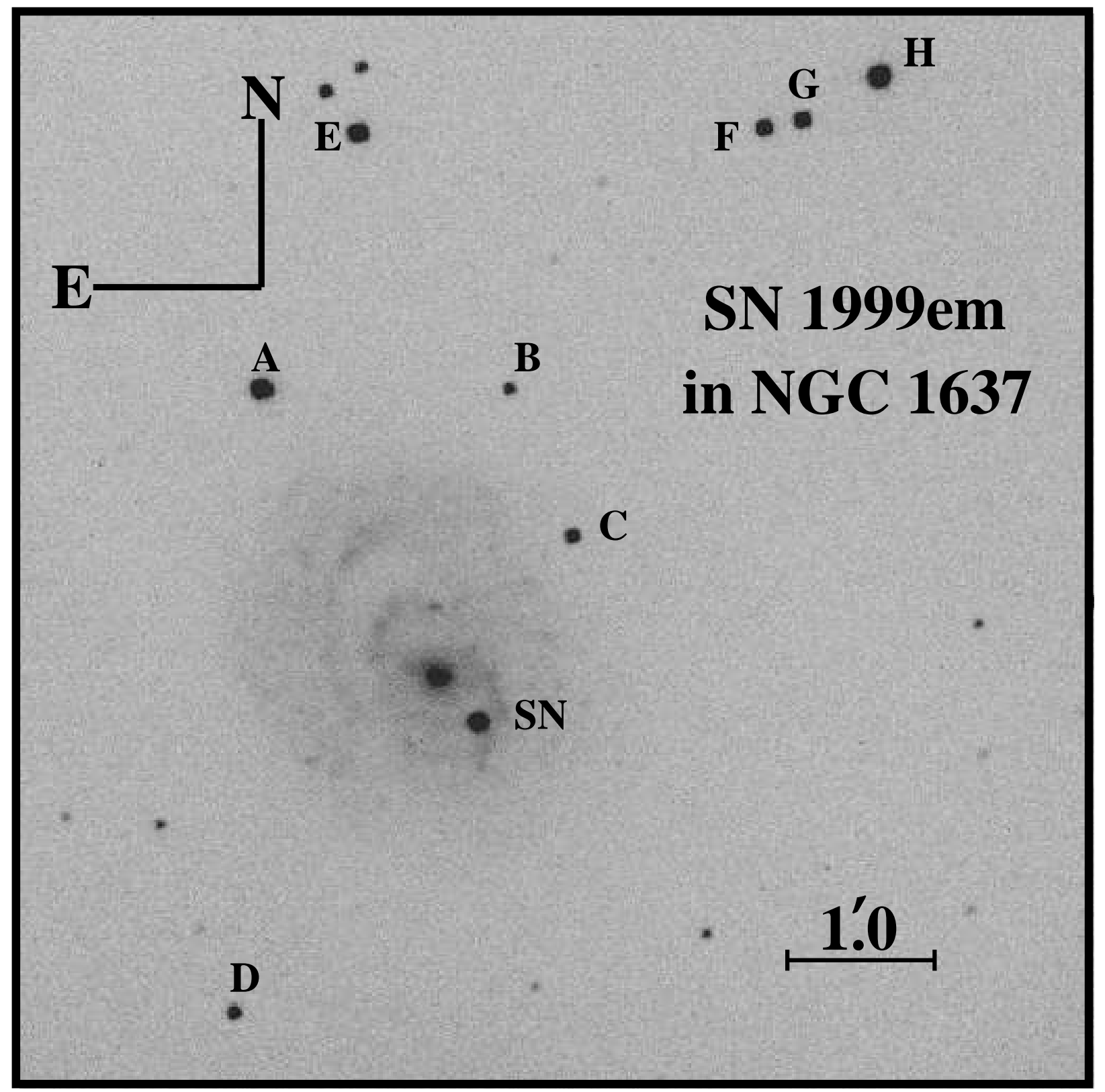

Fig. 2.- B-band image of NGC 1637 taken on 1999 November 1 with the Katzman Automatic Imaging Telescope. SN $1999 \mathrm{em}(S N)$ is measured to be $15^{\prime \prime} 1$ west and $17^{\prime \prime} .2$ south of the galaxy nucleus (cf. Jha et al. 1999). The local standards listed in Table 1 are marked. 
Table 1. Magnitudes of Local Standards

\begin{tabular}{lccccc}
\hline \hline Star & $U$ & $B$ & $V$ & $R$ & $I$ \\
\hline $\mathrm{A}$ & 14.97 & 13.63 & 12.42 & 11.75 & 11.17 \\
$\mathrm{~B}$ & $\cdots$ & 16.75 & 15.77 & 15.18 & 14.69 \\
$\mathrm{C}$ & 15.66 & 15.74 & 15.12 & 14.76 & 14.38 \\
$\mathrm{D}$ & 15.96 & 16.21 & 15.61 & 15.25 & 14.89 \\
$\mathrm{E}$ & 15.44 & 14.16 & 12.99 & 12.37 & 11.84 \\
$\mathrm{~F}$ & 15.21 & 14.99 & 14.30 & 13.94 & 13.59 \\
$\mathrm{G}$ & $\ldots$ & 14.22 & 13.50 & 13.09 & 12.72 \\
$\mathrm{H}$ & 13.85 & 13.51 & 12.79 & 12.39 & 12.04 \\
\hline
\end{tabular}


Richmond et al. 1995), leaving a "clean" image of the SN with no galaxy contamination. However, nearly a year after discovery SN1999em still remained quite detectable in 10-min KAIT exposures, especially in the $V R I$ bandpasses. Fortunately, during the period of main interest to this study (the first 100 days), SN 1999em was over 6 mag brighter than the galaxy background, reducing uncertainty due to improper background removal to at most the few percent level (e.g., Suntzeff et al. 1999). To obtain the most accurate photometric measurements without the benefits of galaxy subtraction, we used point-spread function (PSF) fitting by determining a PSF for each image with DAOPHOT using bright (but unsaturated) isolated stars in the field. We used only the inner core of SN 1999em and the local standards to fit the PSF in order to reduce errors that can be introduced when there is a strong gradient in the background (e.g., Schmidt et al. 1993). In practice, this core was generally set to be about the FWHM of a given image.

One advantage of using the PSF fitting technique over simple aperture photometry is that it produces an image with the SN and local standard stars subtracted away, which allows a visual check on whether the brightness of the SN has been accurately measured. While the fitting radius of the SN and comparison stars was varied from night to night to match the seeing, the sky background of the SN and local standards was always set to an annulus with a radius of $20-26$ pixels $\left(166^{\prime \prime} 0-20 . \prime 8\right)$ to maintain consistency throughout the observations. We then subtracted the mode of the sky background ${ }^{18}$ to derive the instrumental magnitudes for the SN and local standards, and transformed them to the standard Johnson-Cousins system by taking the weighted mean of the values obtained using the three calibrator stars. Due to the faintness of the field stars in the $U$ band, only star $A$ was used to transform the $U$-band magnitudes. This limitation for $U$, coupled with the general difficulty of calibrating the $U$ band using traditional CCDs with poor blue response (see, e.g., Suntzeff 2000), makes us regard the $U$ photometry as systematically suspect at the $0.1-0.2 \mathrm{mag}$ level.

The results of our photometric observations are given in Table 2 and shown in Figures 3 and 4. The reported uncertainties come from two sources. First, there is the photometric uncertainty reported by the error analysis package in DAOPHOT from the statistics of the SN and background region. Second, there is uncertainty in the transformation to the standard system. We estimate the transformation error by taking the standard deviation of the spread of the standard magnitudes obtained using each of the local standard stars. Since three values do not determine a robust standard deviation, we opted to take a conservative estimate of the transformation error by measuring the standard deviation of the values obtained from all eight of the stars labeled in Figure 2. Due to the faintness of the local standards in the $U$ band, the transformation error in that bandpass was generally obtained using fewer stars. The photometric and transformation errors were then added in quadrature to obtain the uncertainty reported in Table 2; in most cases, the total error was dominated by the uncertainty in the transformation.

\footnotetext{
${ }^{18}$ See Da Costa (1992) for a discussion of the advantages of using the mode of the background region rather than the mean or median.
} 
Table 2. Photometric Observations of SN 1999em

\begin{tabular}{|c|c|c|c|c|c|c|}
\hline UT Date $^{\mathrm{a}}$ & Day $^{b}$ & $U\left(\sigma_{U}\right)$ & $B\left(\sigma_{B}\right)$ & $V\left(\sigma_{V}\right)$ & $R\left(\sigma_{R}\right)$ & $I\left(\sigma_{I}\right)$ \\
\hline 1999-10-30 & 1.0 & $13.03(0.27)$ & $13.87(0.02)$ & $13.87(0.01)$ & $13.67(0.02)$ & $13.65(0.08)$ \\
\hline 1999-10-31 & 2.0 & $13.00(0.11)$ & $13.80(0.03)$ & $13.80(0.02)$ & $13.62(0.03)$ & $13.58(0.03)$ \\
\hline 1999-11-01 & 3.0 & $13.03(0.12)$ & $13.80(0.02)$ & $13.79(0.02)$ & $13.60(0.02)$ & $13.56(0.03)$ \\
\hline 1999-11-02 & 4.0 & $13.11(0.09)$ & $13.82(0.03)$ & $13.79(0.02)$ & $13.59(0.02)$ & $13.56(0.03)$ \\
\hline 1999-11-03 & 5.0 & $13.16(0.14)$ & $13.85(0.02)$ & $13.79(0.01)$ & $13.57(0.02)$ & $13.54(0.02)$ \\
\hline 1999-11-05 & 7.0 & $13.29(0.09)$ & $13.92(0.03)$ & $13.84(0.01)$ & $13.59(0.02)$ & $13.53(0.03)$ \\
\hline 1999-11-06 & 8.0 & $13.34(0.09)$ & $13.95(0.02)$ & $13.84(0.01)$ & $13.58(0.02)$ & $13.50(0.03)$ \\
\hline 1999-11-07 & 9.0 & $13.41(0.09)$ & $13.99(0.01)$ & $13.86(0.02)$ & $13.58(0.02)$ & $13.51(0.02)$ \\
\hline 1999-11-09 & 11.0 & & $14.02(0.04)$ & $13.84(0.04)$ & $13.54(0.05)$ & $13.48(0.04)$ \\
\hline $1999-11-10^{c}$ & 12.0 & $\ldots$ & $14.07(0.15)$ & $13.83(0.10)$ & & \\
\hline 1999-11-11 & 13.0 & $13.90(0.11)$ & $14.09(0.01)$ & $13.80(0.01)$ & $13.53(0.02)$ & $13.44(0.02)$ \\
\hline 1999-11-12 & 14.0 & $14.02(0.14)$ & $14.15(0.01)$ & $13.81(0.01)$ & $13.52(0.02)$ & $13.43(0.02)$ \\
\hline 1999-11-13 & 15.0 & $14.18(0.12)$ & $14.20(0.01)$ & $13.81(0.02)$ & $13.53(0.01)$ & $13.42(0.01)$ \\
\hline 1999-11-14 & 16.0 & $14.37(0.12)$ & $14.25(0.02)$ & $13.81(0.04)$ & $13.56(0.06)$ & $13.44(0.03)$ \\
\hline 1999-11-16 & 18.0 & $14.56(0.10)$ & $14.34(0.02)$ & $13.85(0.03)$ & $13.56(0.07)$ & $13.44(0.06)$ \\
\hline 1999-11-19 & 21.0 & $14.97(0.11)$ & $14.47(0.02)$ & $13.86(0.03)$ & $13.56(0.03)$ & $13.40(0.03)$ \\
\hline 1999-11-26 & 28.0 & $15.46(0.13)$ & $14.73(0.02)$ & $13.91(0.02)$ & $13.57(0.02)$ & $13.35(0.02)$ \\
\hline 1999-11-28 & 30.0 & $15.56(0.16)$ & $14.79(0.04)$ & $13.94(0.03)$ & $13.59(0.02)$ & $13.35(0.04)$ \\
\hline 1999-12-02 & 34.0 & $15.77(0.10)$ & $14.87(0.03)$ & $13.93(0.02)$ & $13.56(0.02)$ & $13.31(0.02)$ \\
\hline 1999-12-04 & 36.0 & $15.81(0.12)$ & & $13.90(0.03)$ & $13.54(0.02)$ & $13.29(0.02)$ \\
\hline 1999-12-06 & 38.0 & $15.96(0.13)$ & $14.94(0.02)$ & $13.93(0.02)$ & $13.55(0.03)$ & $13.29(0.02)$ \\
\hline 1999-12-08 & 39.9 & $16.02(0.09)$ & $14.97(0.02)$ & $13.93(0.02)$ & $13.54(0.02)$ & $13.28(0.03)$ \\
\hline $1999-12-11^{\mathrm{d}}$ & 42.9 & $16.21(0.11)$ & $14.98(0.04)$ & $13.91(0.03)$ & $13.51(0.03)$ & $13.22(0.05)$ \\
\hline 1999-12-14 & 45.9 & $16.31(0.15)$ & $15.04(0.04)$ & $13.93(0.03)$ & $13.50(0.03)$ & $13.23(0.04)$ \\
\hline $1999-12-15$ & 46.9 & $16.34(0.10)$ & $15.05(0.03)$ & $13.93(0.04)$ & $13.52(0.02)$ & $13.22(0.04)$ \\
\hline 1999-12-18 & 49.9 & $16.45(0.11)$ & $15.09(0.03)$ & $13.94(0.02)$ & $13.50(0.03)$ & $13.21(0.03)$ \\
\hline 1999-12-29 & 60.9 & $16.96(0.13)$ & $15.27(0.02)$ & $13.99(0.02)$ & $13.50(0.03)$ & $13.19(0.04)$ \\
\hline $2000-01-03^{\mathrm{e}}$ & 65.9 & $16.96(0.25)$ & $15.32(0.03)$ & $14.02(0.04)$ & $13.51(0.02)$ & $13.17(0.07)$ \\
\hline 2000-01-08 & 70.8 & $17.01(0.10)$ & $15.38(0.02)$ & $14.03(0.02)$ & $13.50(0.02)$ & $13.22(0.03)$ \\
\hline 2000-01-13 & 75.8 & $17.26(0.16)$ & $15.40(0.04)$ & $14.04(0.03)$ & $13.51(0.02)$ & $13.22(0.03)$ \\
\hline 2000-01-14 & 76.8 & $17.33(0.15)$ & $\ldots$ & $\ldots$ & $\ldots$ & $\ldots$ \\
\hline $2000-01-15^{\mathrm{f}}$ & 77.8 & $\ldots$ & $15.52(0.09)$ & $14.07(0.05)$ & $\ldots$ & $13.23(0.09)$ \\
\hline $2000-01-27$ & 89.8 & $\ldots$ & $15.63(0.05)$ & $14.17(0.01)$ & $13.60(0.04)$ & $13.31(0.03)$ \\
\hline 2000-02-02 & 95.8 & $\ldots$ & $15.74(0.02)$ & $14.27(0.02)$ & $13.69(0.04)$ & $13.38(0.03)$ \\
\hline
\end{tabular}


Table 2. Photometric Observations of SN 1999em - Continued

\begin{tabular}{lcccccc}
\hline \hline UT Date $^{\mathrm{a}}$ & Day $^{\mathrm{b}}$ & $U\left(\sigma_{U}\right)$ & $B\left(\sigma_{B}\right)$ & $V\left(\sigma_{V}\right)$ & $R\left(\sigma_{R}\right)$ & $I\left(\sigma_{I}\right)$ \\
\hline $2000-02-07$ & 100.8 & $\ldots$ & $15.87(0.03)$ & $14.38(0.02)$ & $13.77(0.02)$ & $13.46(0.04)$ \\
$2000-03-01^{\mathrm{d}}$ & 123.8 & $\ldots$ & $\ldots$ & $\ldots$ & $15.04(0.12)$ & $\ldots$ \\
$2000-03-04$ & 126.8 & $\ldots$ & $17.77(0.05)$ & $16.25(0.03)$ & $15.27(0.03)$ & $\ldots$ \\
$2000-03-11$ & 133.7 & $\ldots$ & $17.99(0.05)$ & $16.37(0.03)$ & $15.40(0.04)$ & $14.93(0.07)$ \\
$2000-03-16$ & 138.7 & $\ldots$ & $17.97(0.09)$ & $16.42(0.03)$ & $15.42(0.03)$ & $14.93(0.03)$ \\
$2000-03-21^{\mathrm{f}}$ & 143.7 & $\ldots$ & $18.06(0.12)$ & $16.36(0.05)$ & $15.41(0.03)$ & $14.93(0.04)$ \\
$2000-03-26$ & 148.8 & $\ldots$ & $18.10(0.10)$ & $16.49(0.04)$ & $15.47(0.03)$ & $15.01(0.03)$ \\
$2000-04-03$ & 156.8 & $\ldots$ & $17.98(0.09)$ & $16.55(0.04)$ & $15.61(0.03)$ & $15.11(0.03)$ \\
$2000-08-25$ & 301.1 & $\ldots$ & $\ldots$ & $\ldots$ & $16.79(0.06)$ & $\ldots$ \\
$2000-09-09$ & 316.1 & $\ldots$ & $\ldots$ & $\ldots$ & $16.82(0.06)$ & $16.31(0.07)$ \\
$2000-09-16$ & 323.1 & $\ldots$ & $\ldots$ & $\ldots$ & $16.81(0.06)$ & $16.37(0.08)$ \\
$2000-09-23$ & 330.1 & $\ldots$ & $\ldots$ & $\ldots$ & $16.85(0.06)$ & $16.34(0.07)$ \\
$2000-09-25$ & 332.1 & $\ldots$ & $18.79(0.08)$ & $17.84(0.07)$ & $16.97(0.06)$ & $16.39(0.06)$ \\
$2000-10-01$ & 338.1 & $\ldots$ & $\ldots$ & $\ldots$ & $17.05(0.08)$ & $16.52(0.09)$ \\
$2000-10-13$ & 350.0 & $\ldots$ & $\ldots$ & $\ldots$ & $17.15(0.07)$ & $16.63(0.08)$ \\
\hline
\end{tabular}

ayyyy-mm-dd.

${ }^{\mathrm{b}}$ Day since discovery, 1999-10-29 UT (HJD 2,451,480.94).

${ }^{\mathrm{c}}$ Cloudy.

dPoor seeing $\left(z 6^{\prime \prime}\right)$.

${ }^{\text {e}}$ Poor guiding - images trailed.

${ }^{\mathrm{f}}$ Bright moon. 


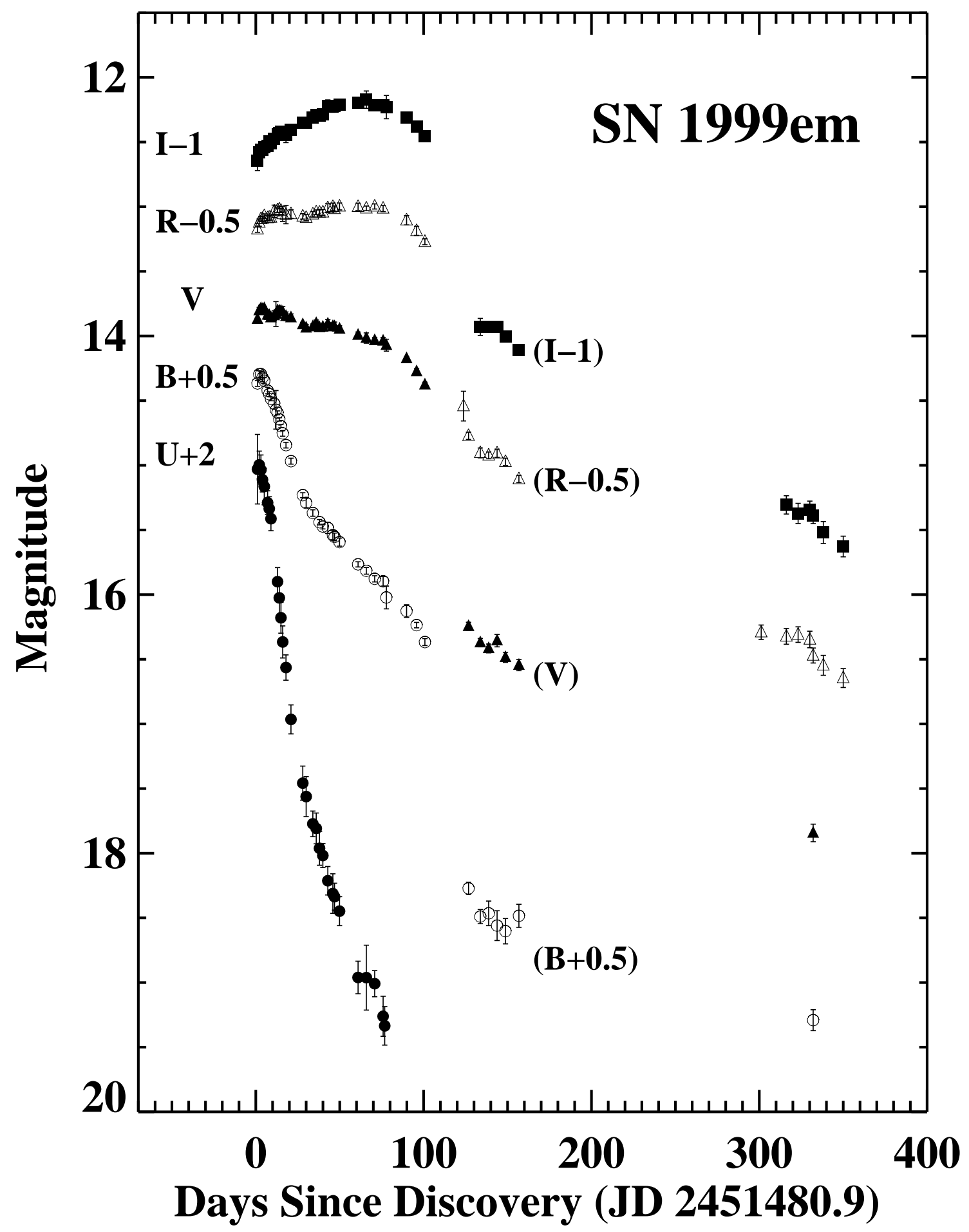

Fig. 3.- UBVRI light curves for SN 1999em from Table 2. For clarity, the magnitude scales for $U B R I$ have been shifted by the amounts indicated. 


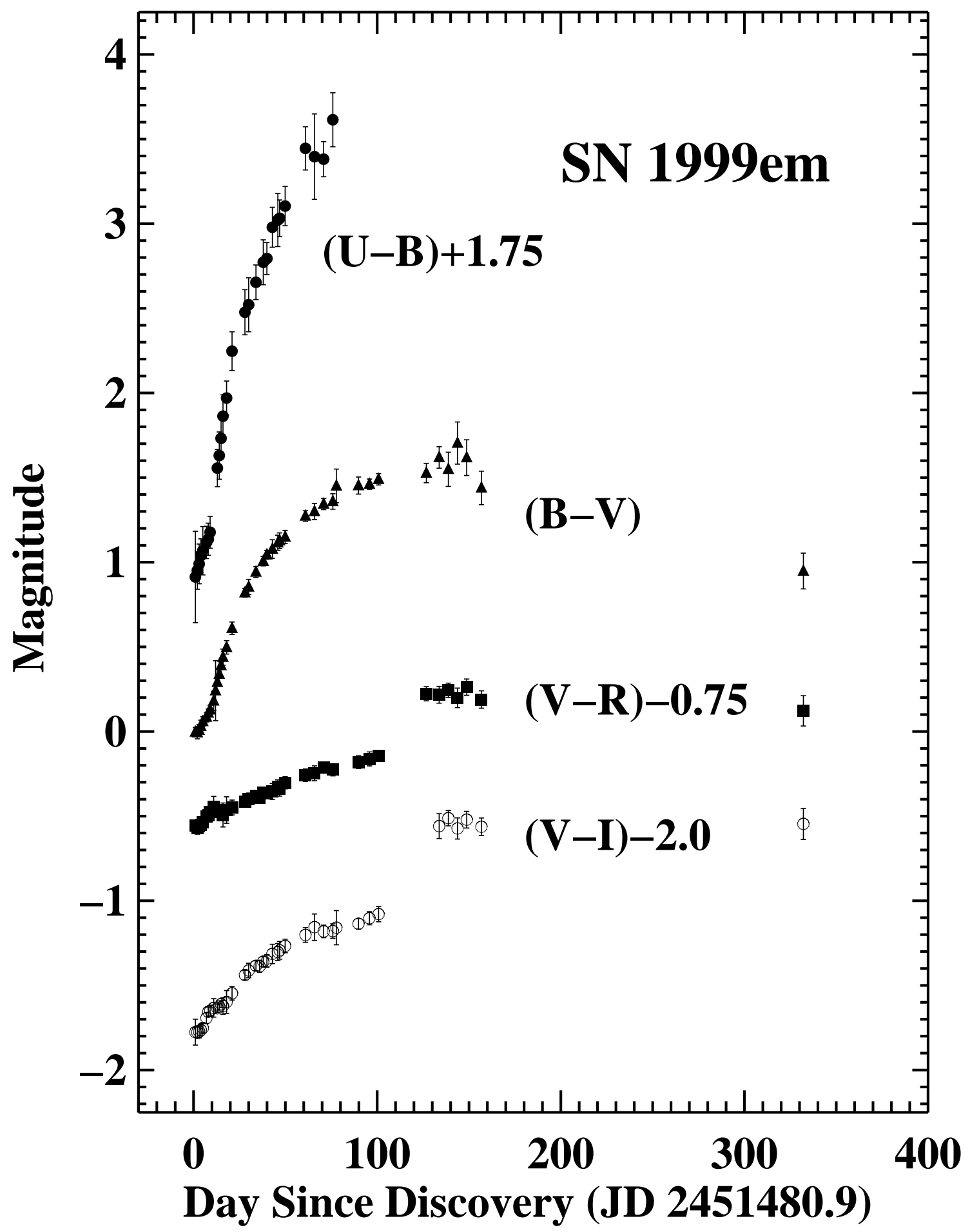

Fig. 4.- $U-B, B-V, V-R$, and $V-I$ color curves of SN 1999em. The magnitude scales for $U-B, V-R, V-I$ have been shifted by the amounts indicated. 
The VRI light curves of SN 1999em shown in Figure 3 indicate a clear plateau lasting until about 95 days from discovery. The plateau phase is characterized by a steeply declining $U$-band brightness, a declining $B$-band brightness, a slightly declining $V$-band brightness, a nearly constant $R$-band brightness, and an $I$-band brightness that increases through the first 70 days and then slowly declines for the remainder of the plateau. The $U$-band and $B$-band light curves decline by an average of 0.06 and $0.02 \mathrm{mag}$ day $^{-1}$, respectively, during the plateau epoch after an initial quick rise to maximum on about day 2 after discovery. We measure the decline in the $B$ band over the first 100 days after maximum to be $\beta_{100}^{B}=2.1 \mathrm{mag}$, firmly establishing SN 1999em as a Type II-P event according to the definition of Patat et al. (1994), used to discriminate between SNe II-P $\left(\beta_{100}^{B}<3.5 \mathrm{mag}\right)$ and SNe II-L $\left(\beta_{100}^{B}>3.5 \mathrm{mag}\right)$.

The UBVRI photometric behavior of SN $1999 \mathrm{em}$ is broadly understood by considering the temporal evolution of the radius at the thermalization depth and color temperature shown in Figure 5 (using quantities subsequently derived by the EPM analysis in $\S 3$ ). The surface area at the thermalization depth is seen to increase throughout most of the plateau as the overall expansion of the ejecta competes with the inwardly moving recombination front. If this were the only effect operating, we would expect to observe increasing brightness throughout the majority of the plateau phase. Countering this, however, is the decreasing temperature of the photosphere as the SN cools, which lowers the flux at all wavelengths. The effect of the decreasing photospheric temperature is least pronounced at longer wavelengths (i.e., the $I$ band) since bandpasses significantly redward of the blackbody peak are more nearly in the Rayleigh-Jeans regime of the Planck function and thus only linearly dependent on temperature. As one moves to progressively bluer wavelengths the effect of the decreasing temperature becomes more pronounced, and the plateau brightness changes from generally increasing ( $I$ band) to nearly constant $(R$ band), to slightly declining ( $V$ band), to moderately declining ( $B$ band), to steeply declining ( $U$ band); significant line blanketing by metals further reduces the flux in the $U$ and $B$ bandpasses. The precipitous drop observed in all bandpasses after about day 95 signals the point at which the hydrogen recombination wave has receded completely through the massive hydrogen envelope and the transition to the nebular phase has begun. The full nebular phase, usually defined as the point at which the total electronscattering optical depth to the center drops below unity, is not expected until after about day 250 (Eastman et al. 1994).

One feature of the $V$-band light curve (and possibly $R$ as well) that warrants special comment is the distinct "double peak" evident in the early stages of development. The first peak occurs near day 4 after discovery (perhaps a bit later than the single peaks seen in $U$ and $B$ ) and the second peak of nearly equal brightness occurs on day 13 , with the dip between them reaching a local minimum on day 9 (0.07 mag fainter than the peak brightness). Similar photometric behavior can be discerned in the light curve presented by Schmidt et al. (1993) for the Type II-P SN 1990E, although in that case the second peak is brighter than the first, and occurs about two weeks after the first peak. In both SN 1999em and SN 1990E the rise to the second maximum coincides with the appearance of Fe II absorption lines in the spectrum $(\S 2.2)$ and the point at which the 


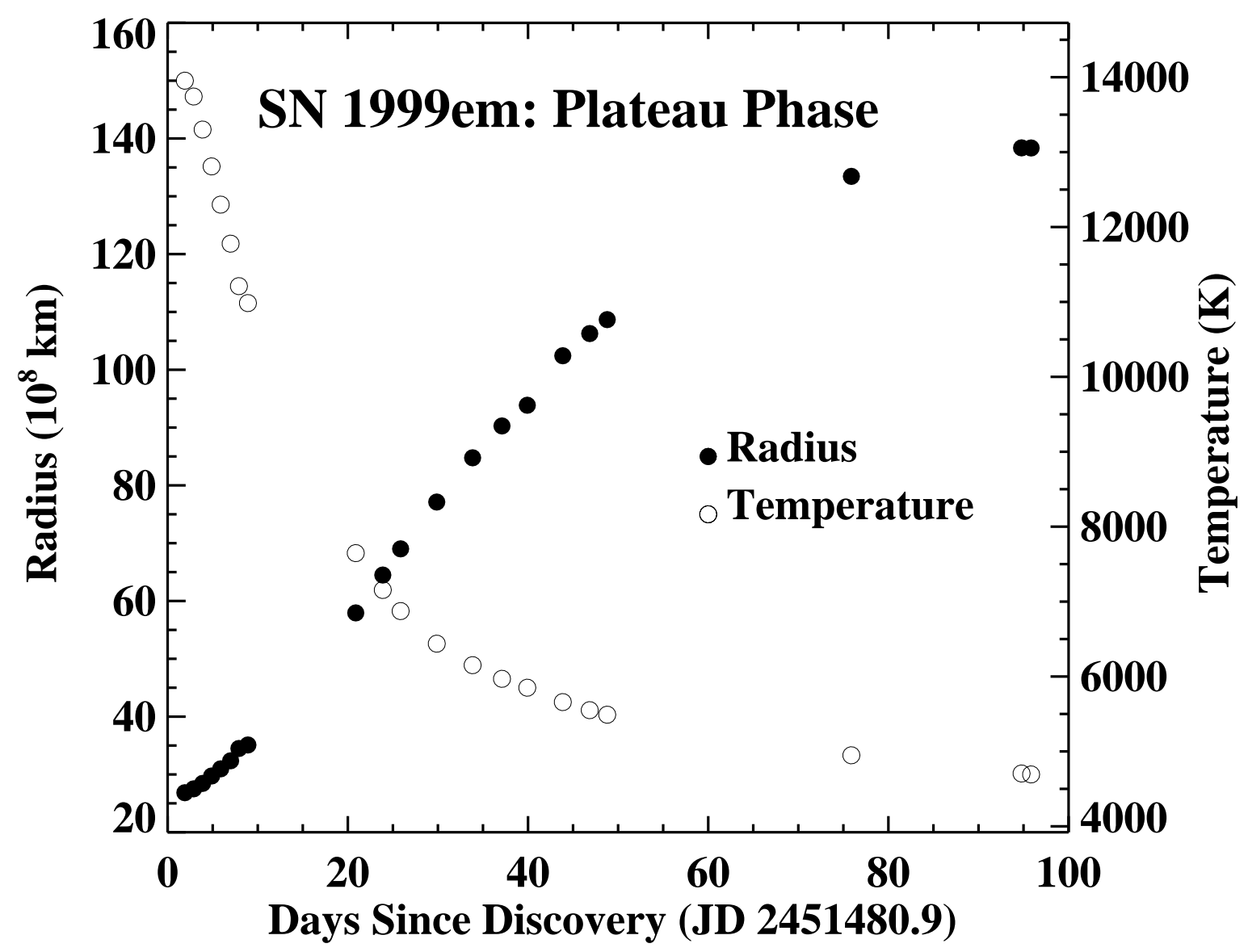

Fig. 5.- The temporal evolution of the radius of the radiating surface at the thermalization depth and $B V I$ color temperature for SN $1999 \mathrm{em}$ during the photospheric phase (i.e., $R=R_{\text {therm }} \equiv \theta \zeta D$ with the theoretical angular size $\left[\theta_{\mathrm{BVI}}\right]$, dilution factor $\left[\zeta_{\mathrm{BVI}}\right]$, and color temperature $\left[T_{\mathrm{BVI}}\right]$ taken from Table 7 , and a distance of $D=D_{\mathrm{BVI}}=8.27 \mathrm{Mpc}$ [Table 8] assumed). 
photospheric color temperature has cooled down to near 10,000 K. This effectively marks the onset of the recombination phase. It is possible that the additional liberation of recombination energy slows the fall of the effective temperature enough to allow the effects of the increasing photospheric surface area to temporarily dominate the visual brightness of the SN. Additional examples of wellsampled early-time SNe II-P light curves are needed to see if this effect is generic.

\subsection{Spectra}

\subsubsection{Observations, Reductions, and Identification of Line Features}

Table 3 lists the spectral observations of SN 1999em. With the exception of the single echelle spectrum, whose reduction details will be given elsewhere (D. C. Leonard et al., in preparation), all one-dimensional sky-subtracted spectra were extracted optimally (Horne 1986) in the usual manner, generally with a width of $\sim 10^{\prime \prime}$ along the slit, although this was modified based on seeing conditions (usually a width of $\sim 4$ times the seeing was employed). Each spectrum was then wavelength and flux calibrated, as well as corrected for continuum atmospheric extinction and telluric absorption bands (Wade \& Horne 1988; Bessell 1999; Matheson et al. 2000).

In general, the position angle of the slit was not aligned along the parallactic angle (the slit of the Lick 1-m spectrograph is fixed at $0^{\circ}$ ) so the spectral shape may suffer from differential light loss (Filippenko 1982). In addition, the shape of all spectra obtained with the Lick 1-m reflector is suspect, especially at wavelengths greater than $5800 \AA$, since successive observations from the same night occasionally showed significant variations. ${ }^{19}$ Multiple extractions of the data using apertures of varying width and different background regions failed to remedy the problem, and its cause remains unknown. Fortunately, this calibration uncertainty has minimal scientific impact here since it is the wavelengths of absorption features, not absolute fluxes, that are of primary interest. In any case, the effect was evident on only a few nights, and quantities derived using spectra obtained on these nights are in good agreement with those derived from spectra taken at other telescopes.

The spectral evolution of SN 1999em during the first 517 days of its development is shown in Figures 6 and 7. The early spectra are characterized by a nearly featureless continuum with broad hydrogen Balmer and He I $\lambda 5876$ P-Cygni lines. As early as day 7, a hint of Fe II $\lambda 5169$ absorption is visible, and it becomes quite strong by day 11 along with Fe II $\lambda 5018$. During the next 90 days the strength of numerous metal lines continues to increase (see Figure 1). After the plateau ends, the spectrum becomes more emission dominated and marked by the presence of such forbidden lines as [Ca II] $\lambda \lambda 7291,7324$ and [O I] $\lambda \lambda 6300,6364$.

\footnotetext{
${ }^{19}$ Normalizing the spectrum at the blue end, the flux level of the red end varied by as much as $\pm 30 \%$ on the worst nights.
} 
Table 3. Journal of Spectroscopic Observations of SN 1999em

\begin{tabular}{|c|c|c|c|c|c|c|c|c|c|c|c|c|}
\hline Day $^{a}$ & $\begin{array}{c}\text { UT Date } \\
\text { (yyyy-mm-dd) }\end{array}$ & Tel. $^{\mathrm{b}}$ & $\begin{array}{c}\text { Range }^{\mathrm{c}} \\
(\AA)\end{array}$ & $\begin{array}{l}\text { Res. }^{\mathrm{d}} \\
(\AA)\end{array}$ & $\begin{array}{l}\text { P.A. } \\
\text { (deg) }\end{array}$ & $\begin{array}{l}\text { Opt. P.A. } \\
\text { (deg) }\end{array}$ & Air. ${ }^{g}$ & Flux Std. ${ }^{\mathrm{h}}$ & $\begin{array}{c}\text { See }{ }^{\mathrm{i}} \\
(\operatorname{arcsec})\end{array}$ & $\begin{array}{c}\text { Slit } \\
(\operatorname{arcsec})\end{array}$ & $\begin{array}{l}\text { Exp. } \\
\text { (s) }\end{array}$ & Observer $(\mathrm{s})^{\mathrm{j}}$ \\
\hline 1.9 & $1999-10-31$ & L1 & 3948-7449 & 6 & 0 & 150 & 1.5 & HD19 & 2.0 & 2.9 & $2 \times 900$ & EG \\
\hline 2.9 & 1999-11-01 & L1 & 3948-7449 & 7 & 0 & 147 & 1.5 & HD19 & 2.2 & 2.9 & $3 \times 900$ & EG \\
\hline 3.9 & 1999-11-02 & L1 & 3948-7449 & 7 & 0 & 154 & 1.6 & HD19 & 1.4 & 2.9 & $2 \times 900$ & $\mathrm{EG}$ \\
\hline 4.0 & 1999-11-02 & L3h & $3630-10100$ & 0.1 & $\ldots$ & $\ldots$ & 1.3 & $\ldots$ & 2.2 & 2.0 & 5400 & $\mathrm{AQ}, \mathrm{SF}, \mathrm{CG}$ \\
\hline 4.9 & 1999-11-03 & L1 & 3948-7449 & 6 & 0 & 155 & 1.5 & HD19 & 2.4 & 2.9 & $3 \times 900$ & $\mathrm{EG}$ \\
\hline 5.9 & 1999-11-04 & L1 & 3948-7449 & 6 & 0 & 159 & 1.4 & HD19 & 1.8 & 2.9 & $3 \times 900$ & EG \\
\hline \multirow[t]{4}{*}{7.0} & 1999-11-05 & L3p & $3400-5500$ & 5 & 170 & 174 & 1.4 & F34 & 2.3 & 2.0 & $4 \times 1200$ & $\mathrm{AF}, \mathrm{DL}, \mathrm{RC}$ \\
\hline & & & $5000-7700$ & 6 & 170 & 174 & 1.4 & HD84 & 2.3 & 2.0 & $4 \times 1200$ & $\mathrm{AF}, \mathrm{DL}, \mathrm{RC}$ \\
\hline & & & $3400-5500$ & 5 & 20 & 24 & 1.5 & F34 & 2.3 & 2.0 & $4 \times 1500$ & $\mathrm{AF}, \mathrm{DL}, \mathrm{RC}$ \\
\hline & & & $5000-7700$ & 6 & 20 & 24 & 1.5 & HD84 & 2.3 & 2.0 & $4 \times 1500$ & $\mathrm{AF}, \mathrm{DL}, \mathrm{RC}$ \\
\hline 7.9 & 1999-11-06 & L1 & $3948-7449$ & 6 & 0 & 157 & 1.4 & HD19 & 2.1 & 2.9 & $3 \times 900$ & EG \\
\hline 8.9 & 1999-11-07 & L1 & 3948-7449 & 8 & 0 & 158 & 1.4 & HD19 & 4.2 & 2.9 & $3 \times 900$ & EG \\
\hline 10.2 & 1999-11-08 & KII & $4000-10000$ & 14 & 63 & 61 & 1.6 & BD17 & 1 & 0.7 & $30+70+70$ & $\mathrm{AF}, \mathrm{AC}, \mathrm{AR}$ \\
\hline 11.2 & 1999-11-09 & KII & $4000-10000$ & 14 & 58 & 61 & 1.5 & BD17 & 1 & 0.7 & $60+70$ & $\mathrm{AF}, \mathrm{AC}, \mathrm{AR}$ \\
\hline 20.9 & 1999-11-19 & L1 & 3948-7449 & 7 & 0 & 159 & 1.4 & HD19 & 3 & 2.9 & $3 \times 900$ & EG \\
\hline 23.9 & $1999-11-22$ & L1 & 3948-7449 & 7 & 0 & 162 & 1.4 & HD19 & 2.2 & 2.9 & $4 \times 900$ & EG \\
\hline 25.9 & 1999-11-24 & L1 & 3948-7449 & 7 & 0 & 166 & 1.4 & HD19 & 2.7 & 2.9 & $4 \times 900$ & EG \\
\hline 29.9 & $1999-11-28$ & L1 & 3948-7449 & 7 & 0 & 164 & 1.4 & HD19 & 3.0 & 2.9 & $4 \times 900$ & EG \\
\hline 33.9 & 1999-12-02 & $\mathrm{L} 1$ & 3948-7449 & 7 & 0 & 160 & 1.4 & HD19 & 3.5 & 2.9 & $4 \times 900$ & EG \\
\hline 37.1 & 1999-12-05 & KII & $4000-10000$ & 14 & 61 & 61 & 1.0 & HD84 & 2.2 & 0.7 & 330 & $\mathrm{AF}, \mathrm{PG}$ \\
\hline \multirow[t]{2}{*}{39.9} & $1999-12-08$ & L3p & $4230-7010$ & 7 & 16 & 28 & 1.5 & HD84 & 3 & 3.0 & $4 \times 1200$ & $\mathrm{DL}, \mathrm{DA}$ \\
\hline & & & $4230-7010$ & 7 & 43 & 45 & 2.1 & HD84 & 4 & 3.0 & $4 \times 1200$ & $\mathrm{DL}, \mathrm{DA}$ \\
\hline \multirow[t]{2}{*}{43.9} & $1999-12-12$ & L1 & 3948-7449 & 7 & 0 & 177 & 1.4 & HD19 & 2.6 & 2.9 & $3 \times 900$ & EG \\
\hline & & & $5550-8649$ & 7 & 0 & 177 & 1.4 & HD19 & 2.6 & 2.9 & $3 \times 900$ & EG \\
\hline \multirow[t]{2}{*}{46.8} & $1999-12-15$ & L1 & 3948-7449 & 7 & 0 & 177 & 1.4 & HD19 & 2.7 & 2.9 & $3 \times 900$ & EG \\
\hline & & & $5550-8649$ & 7 & 0 & 177 & 1.4 & HD19 & 2.7 & 2.9 & $3 \times 900$ & EG \\
\hline \multirow[t]{2}{*}{48.8} & $1999-12-17$ & L3p & $4220-6882$ & 6 & 161 & 170 & 1.4 & HD19 & 2.3 & 2.0 & $4 \times 1800$ & $\mathrm{DL}, \mathrm{RC}, \mathrm{AC}$ \\
\hline & & & $4220-6882$ & 6 & 24 & 24 & 1.5 & HD19 & 2.5 & 2.0 & $4 \times 1800$ & $\mathrm{DL}, \mathrm{RC}, \mathrm{AC}$ \\
\hline \multirow[t]{4}{*}{75.9} & 2000-01-13 & $\mathrm{P}$ & $3500-5500$ & 11 & 90 & 43 & 1.7 & HD84 & 2.1 & 1.0 & $3 \times 120$ & $\mathrm{AH}, \mathrm{SB}$ \\
\hline & & & 5400-10000 & 14 & 90 & 43 & 1.7 & HD84 & 2.1 & 1.0 & $3 \times 120$ & $\mathrm{AH}, \mathrm{SB}$ \\
\hline & & & $3500-5500$ & 27 & 90 & 43 & 1.7 & HD84 & 2.1 & 6.0 & $2 \times 60$ & $\mathrm{AH}, \mathrm{SB}$ \\
\hline & & & $5400-10000$ & 53 & 90 & 43 & 1.7 & HD84 & 2.1 & 6.0 & $2 \times 60$ & $\mathrm{AH}, \mathrm{SB}$ \\
\hline 94.8 & $2000-02-01$ & KII & $4510-7010$ & 5 & 46 & 141 & 1.3 & G191 & 0.9 & 1.0 & 120 & WB, DS, AS, WV, MR, GM \\
\hline 95.8 & $2000-02-02$ & L1 & 3948-7449 & 6 & 0 & 31 & 1.7 & HD19 & 2.5 & 2.9 & $4 \times 900$ & EG \\
\hline 123.8 & 2000-03-01 & KI & $3700-8500$ & 10 & 0 & 46 & 1.2 & BD26 & 1.0 & 1.0 & 2x60 & $\mathrm{MM}, \mathrm{RE}, \mathrm{JC}$ \\
\hline \multirow[t]{2}{*}{137.7} & 2000-03-15 & L3 & $3400-5400$ & 7 & 33 & 34 & 1.6 & F34 & 3.0 & 2.0 & $800+320$ & $\mathrm{AF}, \mathrm{AC}$ \\
\hline & & & $5100-10500$ & 11 & 33 & 34 & 1.6 & BD26 & 3.0 & 2.0 & $800+320$ & $\mathrm{AF}, \mathrm{AC}$ \\
\hline 158.8 & 2000-04-05 & KIp & $4338-6872$ & 7 & 66 & 66 & 2.3 & HD84 & 1.9 & 1.5 & $3 \times 900$ & $\mathrm{AF}, \mathrm{AB}$ \\
\hline 162.8 & 2000-04-09 & KIp & $3720-8685$ & 13 & 66 & 65 & 2.1 & F34 & 1.5 & 1.5 & $4 \times 300$ & $\mathrm{RB}, \mathrm{MB}, \mathrm{RW}$ \\
\hline 313.1 & 2000-09-06 & L3 & $3400-5400$ & 7 & 150 & 154 & 1.5 & BD28 & 3.0 & 2.0 & $1800+300$ & $\mathrm{AF}, \mathrm{RC}, \mathrm{WL}$ \\
\hline
\end{tabular}


Table 3-Continued

\begin{tabular}{|c|c|c|c|c|c|c|c|c|c|c|c|c|}
\hline Day $^{\mathrm{a}}$ & $\begin{array}{c}\text { UT Date } \\
\text { (yyyy-mm-dd) }\end{array}$ & Tel. $^{\mathrm{b}}$ & $\begin{array}{c}\text { Range }^{\mathrm{c}} \\
(\AA)\end{array}$ & $\begin{array}{l}\text { Res. }^{\mathrm{d}} \\
(\AA)\end{array}$ & $\begin{array}{l}\text { P.A. } \\
(\operatorname{deg})\end{array}$ & $\begin{array}{l}\text { Opt. P.A. }{ }^{f} \\
\text { (deg) }\end{array}$ & Air. ${ }^{g}$ & Flux Std. ${ }^{\mathrm{h}}$ & $\begin{array}{c}\text { See. }^{\mathrm{i}} \\
(\operatorname{arcsec})\end{array}$ & $\begin{array}{c}\text { Slit } \\
(\operatorname{arcsec})\end{array}$ & $\begin{array}{l}\text { Exp. } \\
(\mathrm{s})\end{array}$ & Observer $(\mathrm{s})^{\mathrm{j}}$ \\
\hline \multirow{3}{*}{333.1} & & & 5100-10450 & 11 & 150 & 154 & 1.5 & BD17 & 3.0 & 2.0 & $1800+300$ & $\mathrm{AF}, \mathrm{RC}, \mathrm{WL}$ \\
\hline & 2000-09-26 & L3 & $3400-5400$ & 7 & 180 & 168 & 1.5 & BD28 & 2.0 & 2.0 & 2700 & $\mathrm{AF}, \mathrm{RC}$ \\
\hline & & & 5100-7800 & 7 & 180 & 168 & 1.5 & BD17 & 2.0 & 2.0 & 2700 & $\mathrm{AF}, \mathrm{RC}$ \\
\hline 516.7 & 2001-03-29 & KIp & $4340-6870$ & 7 & 60 & 63 & 1.7 & HD84 & 1.0 & 1.0 & 700 & $\mathrm{AF}, \mathrm{AB}$ \\
\hline
\end{tabular}

${ }^{a}$ Days since discovery, 1999-10-29 (HJD 2,451,480.94), rounded to the nearest tenth of a day.

${ }^{\mathrm{b}} \mathrm{L} 1$ = Lick 1-m/Nickel Reflector + spectrograph (Stone \& Shields 1990); L3(p) = Lick 3-m/Kast Double Spectrograph (Miller \& Stone 1993; 'p' denotes polarimeter attached; 'h' denotes Hamilton echelle spectrograph used instead of the Kast); K I(p) = Keck-I 10-m/Low Resolution Imaging Spectrometer (LRIS [Oke et al. 1995]; 'p' denotes polarimeter attached [Cohen 1996]); K II = Keck-II 10-m/LRIS; P = Palomar 5 m Hale/Double Spectrograph (Oke \& Gunn 1983).

${ }^{\mathrm{c}}$ Observed wavelength range of spectrum. In some cases, the ends are very noisy, and are not shown in the figures.

d Approximate spectral resolution derived from night-sky lines.

e Position angle of the spectrograph slit.

${ }^{\mathrm{f}}$ Optimal parallactic angle (Filippenko 1982) at the midpoint of the exposures.

Average airmass of observations.

${ }^{\mathrm{h}}$ The standard stars are as follows: F34 = Feige 34, BD28 = BD $+28^{\circ} 4211-$ Stone (1977), Massey \& Gronwall (1990); G191 = G191B2B-Massey \& Gronwall (1990); HD19 = HD 19445, HD84 = HD 84937, BD26 = BD+26²606, BD17 = BD+17²411—Oke \& Gunn (1983).

${ }^{i}$ Seeing is estimated from the FWHM of point sources on the CCD chip.

${ }^{\mathrm{j}} \mathrm{AB}=$ Aaron Barth; $\mathrm{AC}=$ Alison Coil; $\mathrm{AF}=$ Alex Filippenko; $\mathrm{AH}=$ Alan Harris; $\mathrm{AQ}=$ Andreas Quirrenbach; AR = Adam Riess; $\mathrm{AS}=\mathrm{Adam}$ Stanford; $\mathrm{CG}=$ Carol Grady; DA = David Ardila; DL = Douglas Leonard; DS = Daniel Stern; EG = Elinor Gates; GM = George Miley; JC = James Colbert; $\mathrm{MB}=$ Michael Brotherton; $\mathrm{MM}=$ Matt Malkan; $\mathrm{MR}=$ Michiel Reuland; $\mathrm{PG}=$ Peter Garnavich; RB = Robert Becker; RC = Ryan Chornock; $\mathrm{RE}=$ Rick Edelson; RW = Richard White; $\mathrm{SB}=$ Schelte Bus; $\mathrm{SF}=$ Sabine Frink; WB = Wil van Breugel; WL = Weidong Li; WV = Willem de Vries 
As discussed in $\S 1$, one area of uncertainty in the application of EPM is estimating the photospheric velocity. To test the consistency of velocities measured from different lines, we shall derive velocities from as many features as possible in the optical spectrum of SN 1999em during the photospheric phase. Our goal is to identify several unblended, weak features and compare the velocity derived using them with that obtained from the Fe II $\lambda \lambda 4924,5018,5169$ lines, which have often been used in previous EPM studies.

We first identified 36 distinct absorption features in the optical photospheric spectrum of SN $1999 \mathrm{em}$, focusing mainly on the region between 4000 and $7000 \AA$ since that range was best covered by our spectra. We consulted previously published line identifications (IDs) as a starting point for major features, including Jeffery \& Branch (1990), Williams (1987), Elias et al. (1988), McGregor (1988), Meikle et al. (1993), and Fassia et al. (1998). We then considered the theoretical models by Hatano et al. (1999), in which the spectral signatures of the major ions predicted to occur in SN spectra of all types are calculated (the results of the calculations are displayed in figures retrievable from the authors' web-site $\left.{ }^{20}\right)$. To help estimate the expected relative strengths of the different contributing species, transition wavelengths and oscillator strengths were taken from the Kurucz (1996) spectral line database. We also studied the synthetic SN spectrum of progenitor model s15.44.2 of Weaver \& Woosley $(1993)^{21}$, in which the non-LTE radiative transport code EDDINGTON (Eastman \& Pinto 1993) is used to model the explosion of a $15 M_{\odot}$ red supergiant (RSG) progenitor of solar metallicity 44 days after shock breakout with an effective temperature of $5000 \mathrm{~K}$ and a photosphere located at $5000 \mathrm{~km} \mathrm{~s}^{-1}$. The optical portion of the synthetic spectrum is shown in Figure 8(a), with individual species responsible for many of the line features shown in Figure $8(\mathrm{~b}, \mathrm{c}, \mathrm{d})$. Immediately apparent is that Fe II features dominate the spectrum below $5500 \AA$ and consequently blend with many of the features produced by other ions. Such blended features are in general unreliable for simple velocity measurements since the relative strengths of the species making up the blend vary with photosphere location and temperature. The resulting IDs of the line features are shown in Figures 9 and 10, and all the likely contributors' rest wavelengths, configurations, and energies are listed in Table 4. The IDs for major emission-line features in the nebular stage are shown in Figure 11.

\footnotetext{
${ }^{20}$ http://www.nhn.ou.edu/ baron/papers.html.

${ }^{21}$ See Eastman et al. (1994) for details of the physics involved in the model's calculation; here we are primarily interested in its use to help identify line features.
} 


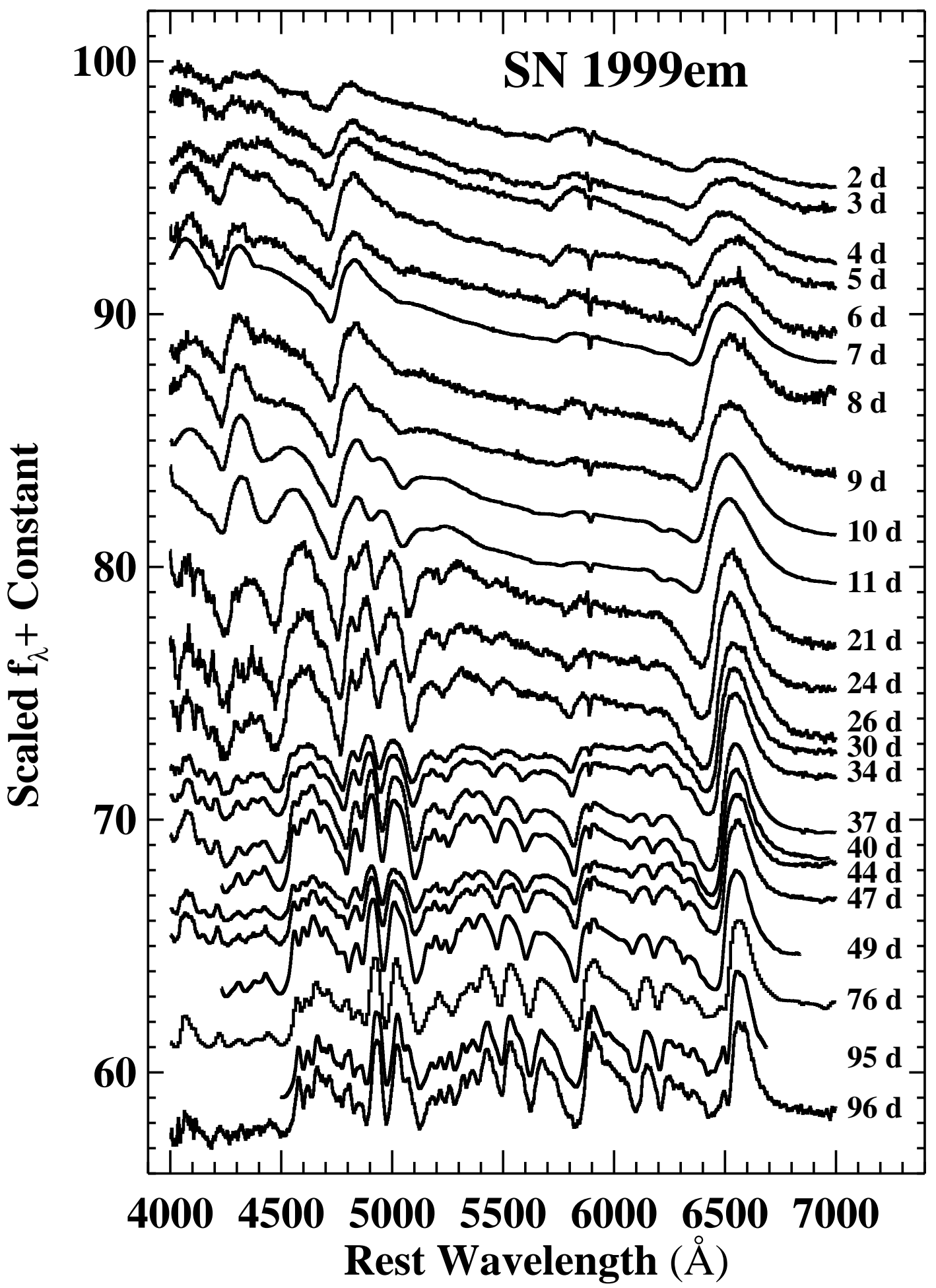

Fig. 6. - Optical flux spectra of SN 1999em spanning the first 96 days after discovery. In this and all figures a recession velocity of $800 \mathrm{~km} \mathrm{~s}^{-1}$ has been removed from the observed spectrum $(\S 2.2 .3)$. Note that the continuum shape of the spectra on days 5 and 6 has been manually adjusted redward of $5800 \AA$ due to irregularities introduced by the spectrograph. 


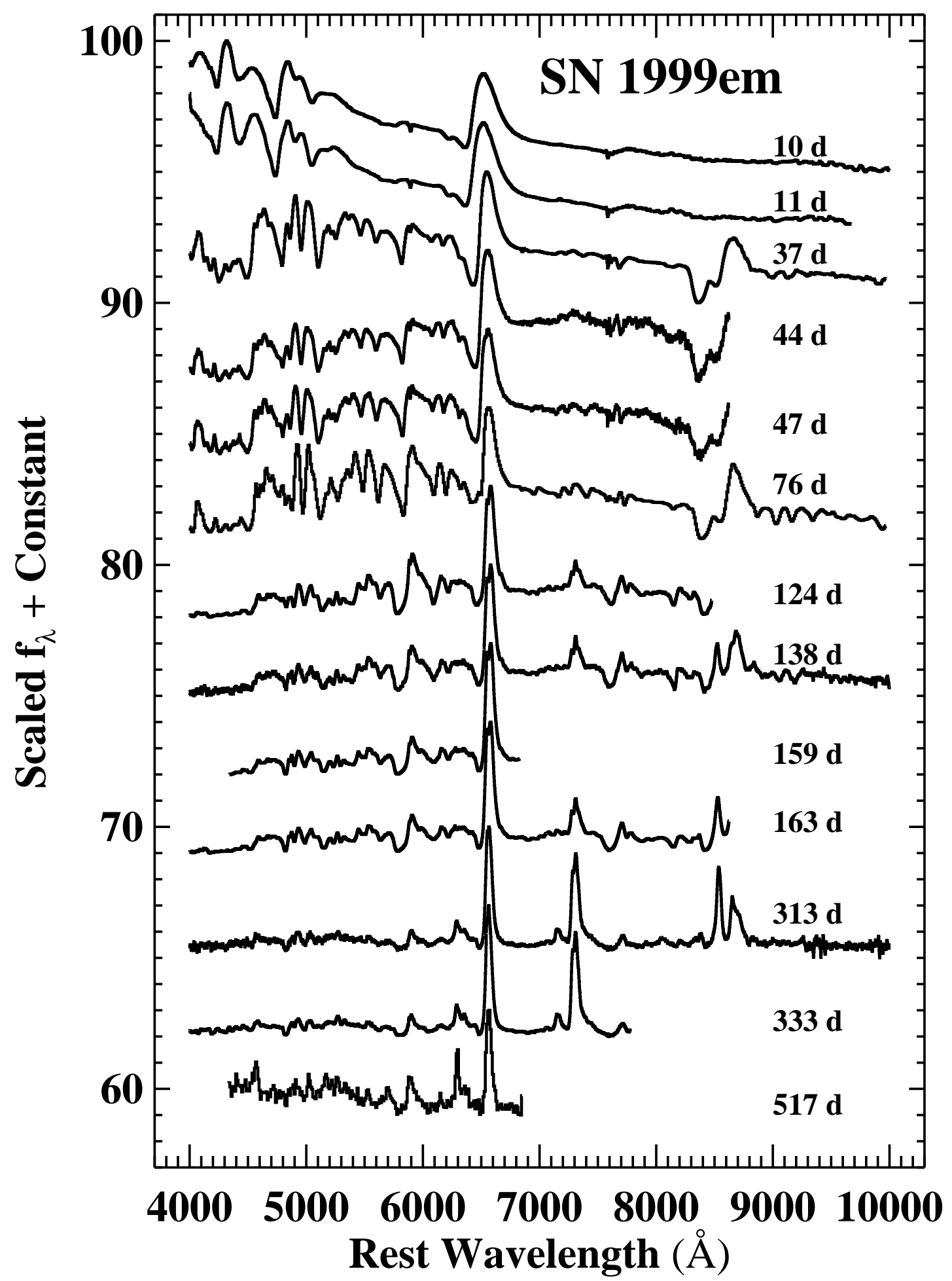

Fig. 7.- The optical and near-infrared spectral development of SN $1999 \mathrm{em}$ during its first 517 days after discovery (1999 October 29). Six plateau-phase spectra shown in Figure 6 are reproduced here as well, but with their full spectral range displayed. 


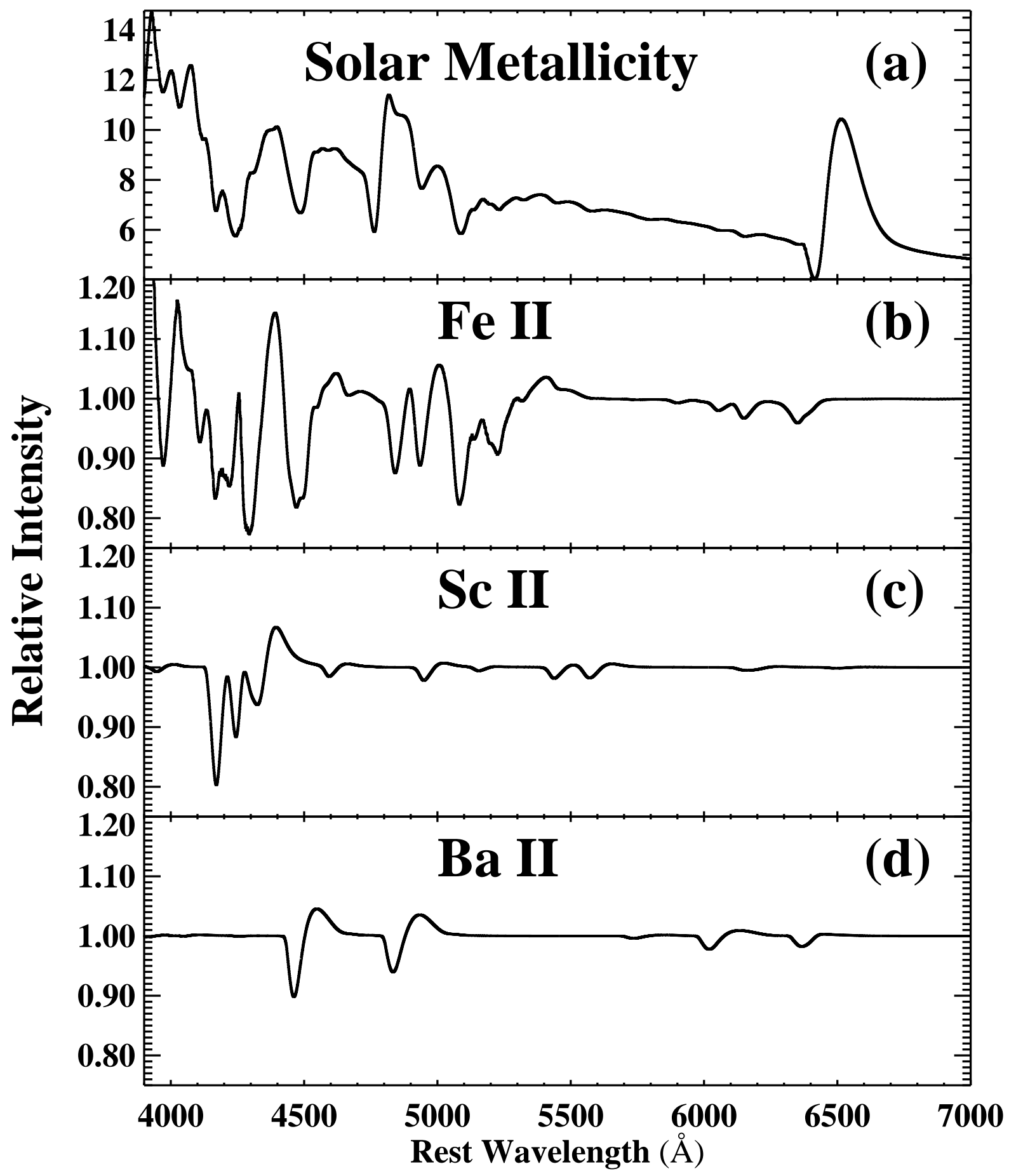

Fig. 8.- (a) Synthetic spectrum of a $15 M_{\odot}$ RSG progenitor with solar metallicity 44 days after explosion with $T_{\text {eff }}=5000 \mathrm{~K}$ (model s15.44.2 of Weaver \& Woosley [1993]). $(b, c, d)$ The relative strengths of line features due to Fe II, Sc II, and Ba II, respectively, in the same model. 


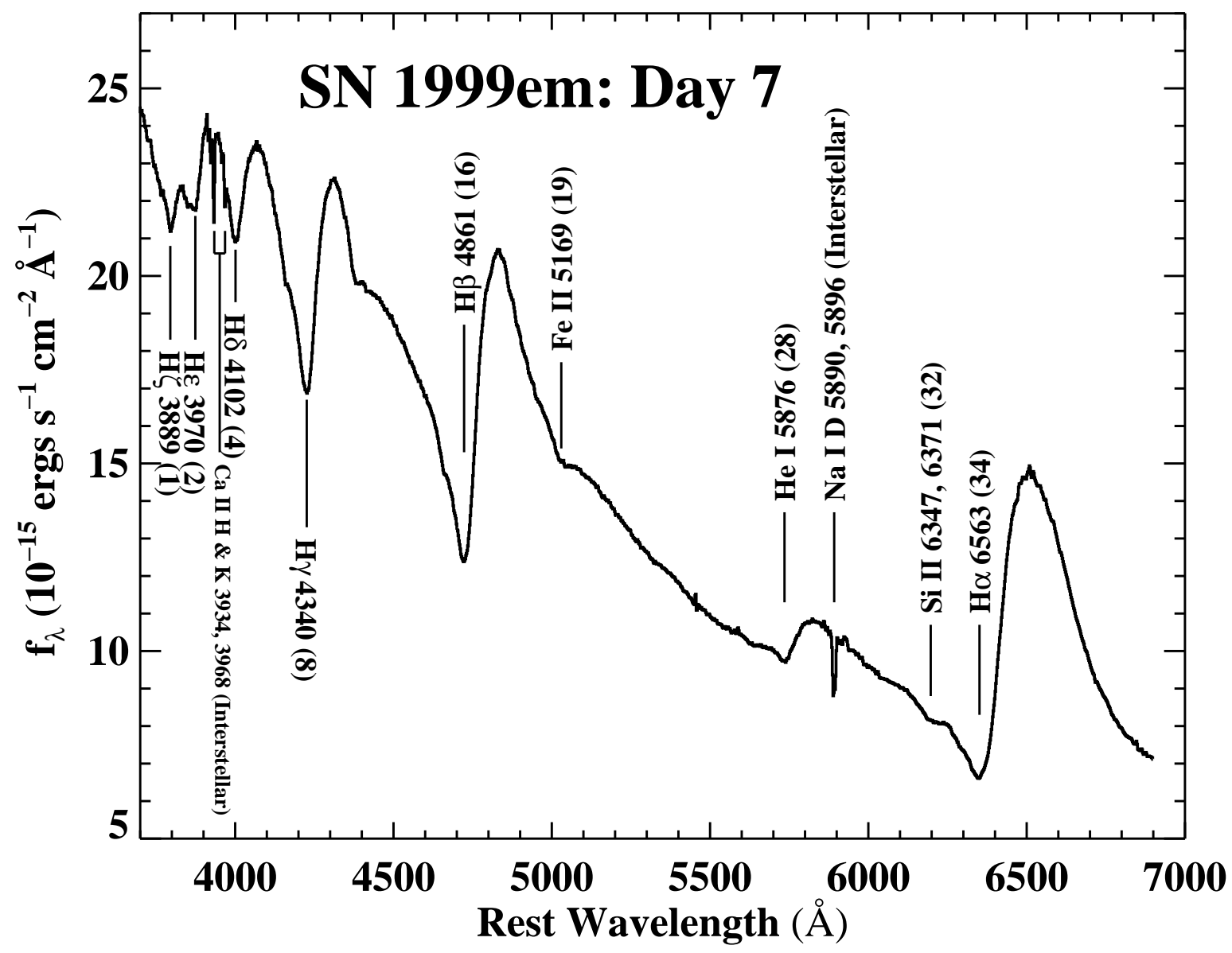

Fig. 9.- Early-time flux spectrum of SN 1999em with prominent absorption features identified; the feature numbers corresponding to the ions listed in Table 4 are given in parenthesis. At this early stage, the only strong lines are due to hydrogen Balmer and He I. Weak Fe II $\lambda 5169$ and Si II $\lambda 6355$ features are also seen. 


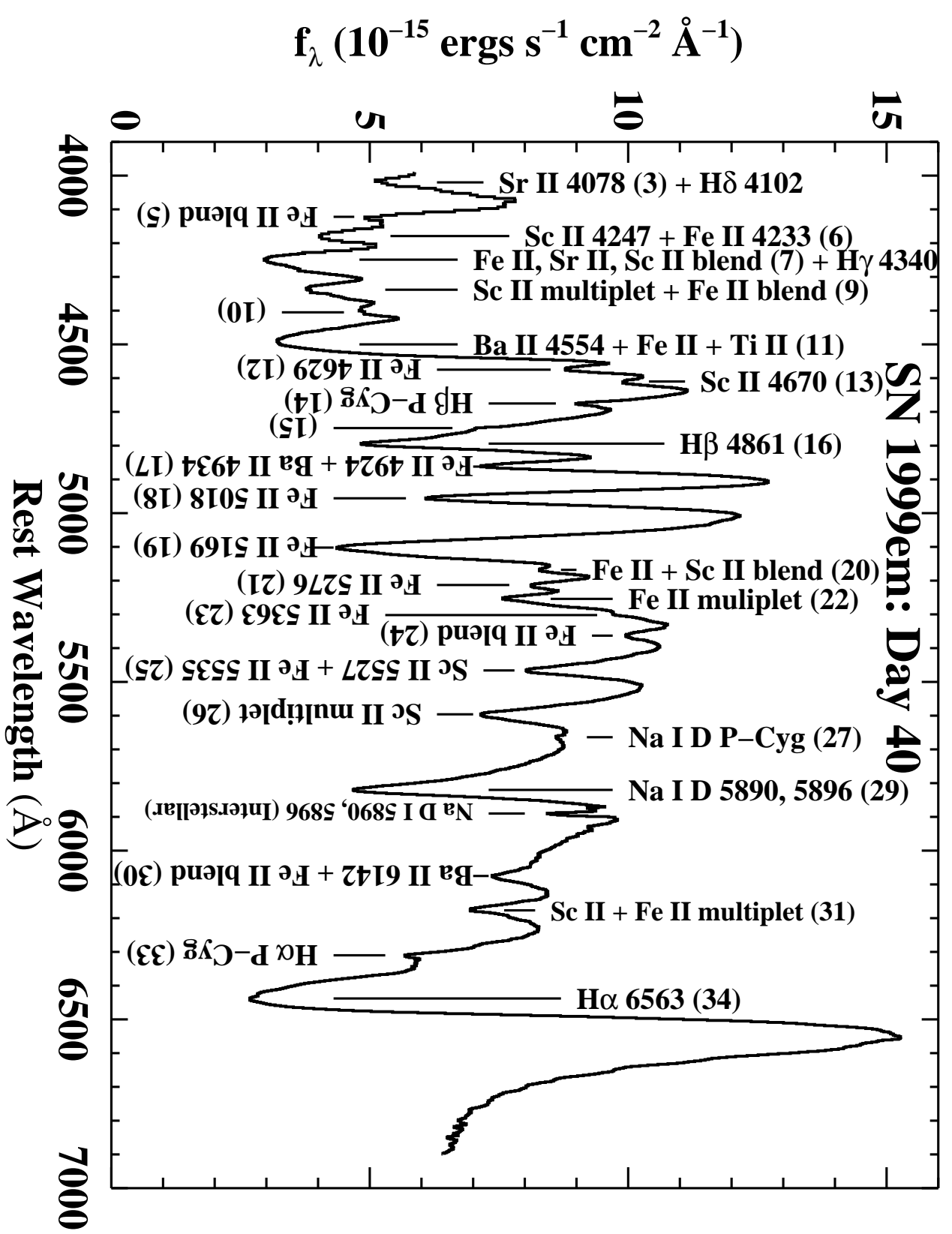


Table 4. SN II-P Photospheric-Phase Absorption Line Identifications

\begin{tabular}{|c|c|c|c|c|c|}
\hline Ion & Wavelength $(\AA)^{\mathrm{a}}$ & $\log (g f)$ & Feature ID $^{\mathrm{b}}$ & Designation & $E(\mathrm{eV})^{\mathrm{c}}$ \\
\hline H I & 3889.05 & -1.19 & 1 & $\ldots d$ & 10.20 \\
\hline H I & 3970.07 & -0.99 & 2 & $\ldots \mathrm{d}$ & 10.20 \\
\hline Sr II & 4077.71 & 0.17 & 3 & $5 s^{2} \mathrm{~S}-5 p^{2} \mathrm{P}^{\circ}$ & 0.00 \\
\hline H I & 4101.73 & -0.75 & 4,3 & $\ldots d$ & 10.20 \\
\hline Fe II & 4173.46 & -2.18 & 5 & $3 d^{6}\left({ }^{3} \mathrm{P} 2\right) 4 s{ }^{4} \mathrm{P}-3 d^{6}\left({ }^{5} \mathrm{D}\right) 4 p^{4} \mathrm{D}^{\circ}$ & 2.58 \\
\hline Fe II & 4178.86 & -2.48 & 5 & $3 d^{6}\left({ }^{3} \mathrm{P} 2\right) 4 s^{4} \mathrm{P}-3 d^{6}\left({ }^{5} \mathrm{D}\right) 4 p{ }^{4} \mathrm{~F}^{\circ}$ & 2.58 \\
\hline Fe II & 4180.98 & -1.84 & 5 & $3 d^{6}\left({ }^{1} \mathrm{D} 2\right) 4 s^{2} \mathrm{D}-3 d^{6}\left({ }^{3} \mathrm{P} 2\right) 4 p^{2} \mathrm{D}^{\circ}$ & 4.74 \\
\hline Fe II & 4233.17 & -2.00 & 6 & $3 d^{6}\left({ }^{3} \mathrm{P} 2\right) 4 s^{4} \mathrm{P}-3 d^{6}\left({ }^{5} \mathrm{D}\right) 4 p^{4} \mathrm{D}^{\circ}$ & 2.58 \\
\hline Sc II & 4246.82 & 0.32 & 6 & $3 d 4 s^{1} \mathrm{D}-3 d 4 p^{1} \mathrm{D}^{\circ}$ & 0.32 \\
\hline Fe II & 4296.57 & -3.01 & 7 & $3 d^{6}\left({ }^{3} \mathrm{P} 2\right) 4 s{ }^{4} \mathrm{P}-3 d^{6}\left({ }^{5} \mathrm{D}\right) 4 p{ }^{4} \mathrm{~F}^{\circ}$ & 2.70 \\
\hline Fe II & 4303.18 & -2.49 & 7 & $3 d^{6}\left({ }^{3} \mathrm{P} 2\right) 4 s^{4} \mathrm{P}-3 d^{6}\left({ }^{5} \mathrm{D}\right) 4 p^{4} \mathrm{D}^{\circ}$ & 2.70 \\
\hline Sr II & 4305.44 & -0.14 & 7 & $5 p^{2} \mathrm{P}-6 s^{2} \mathrm{~S}$ & 3.04 \\
\hline Sc II & 4314.08 & -0.10 & 7 & $3 d^{2}{ }^{3} \mathrm{~F}-3 d 4 p^{3} \mathrm{D}^{\circ}$ & 0.62 \\
\hline Sc II & 4320.73 & -0.26 & 7 & $3 d^{2}{ }^{3} \mathrm{~F}-3 d 4 p^{3} \mathrm{D}^{\circ}$ & 0.61 \\
\hline Sc II & 4325.00 & -0.44 & 7 & $3 d^{2}{ }^{3} \mathrm{~F}-3 d 4 p^{3} \mathrm{D}^{\circ}$ & 0.60 \\
\hline H I & 4340.46 & -0.45 & 8,7 & $\ldots d$ & 10.20 \\
\hline Fe II & 4351.77 & -2.10 & 9 & $3 d^{6}\left({ }^{3} \mathrm{P} 2\right) 4 s^{4} \mathrm{P}-3 d^{6}\left({ }^{5} \mathrm{D}\right) 4 p^{4} \mathrm{D}^{\circ}$ & 2.70 \\
\hline Sc II & 4374.46 & -0.44 & 9 & $3 d^{2}{ }^{3} \mathrm{~F}-3 d 4 p^{3} \mathrm{~F}^{\circ}$ & 0.62 \\
\hline Fe II & 4385.39 & -2.57 & 9 & $3 d^{6}\left({ }^{3} \mathrm{P} 2\right) 4 s^{4} \mathrm{P}-3 d^{6}\left({ }^{5} \mathrm{D}\right) 4 p^{4} \mathrm{D}^{\circ}$ & 2.78 \\
\hline Sc II & 4400.39 & -0.51 & 9 & $3 d^{2}{ }^{3} \mathrm{~F}-3 d 4 p^{3} \mathrm{~F}^{\circ}$ & 0.61 \\
\hline Sc II & 4415.56 & -0.64 & 9 & $3 d^{2}{ }^{3} \mathrm{~F}-3 d 4 p^{3} \mathrm{~F}^{\circ}$ & 0.60 \\
\hline Fe II & 4508.29 & -2.21 & 11 & $3 d^{6}\left({ }^{3} \mathrm{~F} 2\right) 4 s{ }^{4} \mathrm{~F}-3 d^{6}\left({ }^{5} \mathrm{D}\right) 4 p{ }^{4} \mathrm{D}^{\circ}$ & 2.86 \\
\hline Fe II & 4522.63 & -2.03 & 11 & $3 d^{6}\left({ }^{3} \mathrm{~F} 2\right) 4 s{ }^{4} \mathrm{~F}-3 d^{6}\left({ }^{5} \mathrm{D}\right) 4 p{ }^{4} \mathrm{D}^{\circ}$ & 2.84 \\
\hline Fe II & 4549.47 & -1.75 & 11 & $3 d^{6}\left({ }^{3} \mathrm{~F} 2\right) 4 s^{4} \mathrm{~F}-3 d^{6}\left({ }^{5} \mathrm{D}\right) 4 p{ }^{4} \mathrm{D}^{\circ}$ & 2.83 \\
\hline Ti II & 4549.62 & -0.45 & 11 & $3 d^{3}{ }^{2} \mathrm{H}-3 d^{2}\left({ }^{3} \mathrm{~F}\right) 4 p^{2} \mathrm{G}^{\circ}$ & 1.58 \\
\hline Ba II & 4554.03 & 0.17 & 11 & $6 s^{2} \mathrm{~S}-6 p^{2} \mathrm{P}^{\circ}$ & 0.00 \\
\hline Fe II & 4555.89 & -2.29 & 11 & $3 d^{6}\left({ }^{3} \mathrm{~F} 2\right) 4 s{ }^{4} \mathrm{~F}-3 d^{6}\left({ }^{5} \mathrm{D}\right) 4 p{ }^{4} \mathrm{~F}^{\circ}$ & 2.83 \\
\hline Fe II & 4583.84 & -2.02 & 11 & $3 d^{6}\left({ }^{3} \mathrm{~F} 2\right) 4 s^{4} \mathrm{~F}-3 d^{6}\left({ }^{5} \mathrm{D}\right) 4 p{ }^{4} \mathrm{D}^{\circ}$ & 2.81 \\
\hline Fe II & 4629.34 & -2.37 & 12 & $3 d^{6}\left({ }^{3} \mathrm{~F} 2\right) 4 s{ }^{4} \mathrm{~F}-3 d^{6}\left({ }^{5} \mathrm{D}\right) 4 p{ }^{4} \mathrm{~F}^{\circ}$ & 2.81 \\
\hline Sc II & 4670.41 & -0.37 & 13 & $3 d^{2}{ }^{1} \mathrm{D}-3 d 4 p^{1} \mathrm{~F}^{\circ}$ & 1.36 \\
\hline H I & 4861.32 & -0.02 & 16,14 & $\ldots \mathrm{d}^{\mathrm{T}}$ & 10.20 \\
\hline Fe II & 4923.93 & -1.32 & 17 & $3 d^{5} 4 s^{2}{ }^{6} \mathrm{~S}-3 d^{6}\left({ }^{5} \mathrm{D}\right) 4 p{ }^{6} \mathrm{P}^{\circ}$ & 2.89 \\
\hline Ba II & 4934.08 & -0.15 & 17 & $6 s^{2} \mathrm{~S}-6 p^{2} \mathrm{P}^{\circ}$ & 0.00 \\
\hline Fe II & 5018.44 & -1.22 & 18 & $3 d^{5} 4 s^{2}{ }^{6} \mathrm{~S}-3 d^{6}\left({ }^{5} \mathrm{D}\right) 4 p{ }^{6} \mathrm{P}^{\circ}$ & 2.89 \\
\hline
\end{tabular}


Table 4. SN II-P Photospheric-Phase Absorption Line Identifications - Continued

\begin{tabular}{|c|c|c|c|c|c|}
\hline Ion & Wavelength $(\AA)^{\mathrm{a}}$ & $\log (g f)$ & Feature ID $^{\mathrm{b}}$ & Designation & $E(\mathrm{eV})^{\mathrm{c}}$ \\
\hline Sc II & 5031.02 & -0.26 & 18 & $3 d^{2}{ }^{1} \mathrm{D}-3 d 4 p{ }^{1} \mathrm{P}^{\circ}$ & 1.36 \\
\hline Fe II & 5169.03 & -0.87 & 19 & $3 d^{5} 4 s^{2}{ }^{6} \mathrm{~S}-3 d^{6}\left({ }^{5} \mathrm{D}\right) 4 p^{6} \mathrm{P}^{\circ}$ & 2.89 \\
\hline Fe II & 5197.58 & -2.10 & 20 & $3 d^{6}\left({ }^{3} \mathrm{G}\right) 4 s{ }^{4} \mathrm{G}-3 d^{6}\left({ }^{5} \mathrm{D}\right) 4 p{ }^{4} \mathrm{~F}^{\circ}$ & 3.23 \\
\hline Fe II & 5234.62 & -2.05 & 20 & $3 d^{6}\left({ }^{3} \mathrm{G}\right) 4 s^{4} \mathrm{G}-3 d^{6}\left({ }^{5} \mathrm{D}\right) 4 p{ }^{4} \mathrm{~F}^{\circ}$ & 3.22 \\
\hline Sc II & 5239.81 & -0.77 & 20 & $4 s^{2}{ }^{1} \mathrm{~S}-3 d 4 p{ }^{1} \mathrm{P}^{\circ}$ & 1.46 \\
\hline Fe II & 5276.00 & -1.94 & 21 & $3 d^{6}\left({ }^{3} \mathrm{G}\right) 4 s{ }^{4} \mathrm{G}-3 d^{6}\left({ }^{5} \mathrm{D}\right) 4 p{ }^{4} \mathrm{~F}^{\circ}$ & 3.20 \\
\hline Fe II & 5316.62 & -1.85 & 22 & $3 d^{6}\left({ }^{3} \mathrm{G}\right) 4 s^{4} \mathrm{G}-3 d^{6}\left({ }^{5} \mathrm{D}\right) 4 p{ }^{4} \mathrm{~F}^{\circ}$ & 3.15 \\
\hline Fe II & 5325.55 & -2.60 & 22 & $3 d^{6}\left({ }^{3} \mathrm{G}\right) 4 s^{4} \mathrm{G}-3 d^{6}\left({ }^{5} \mathrm{D}\right) 4 p{ }^{4} \mathrm{~F}^{\circ}$ & 3.22 \\
\hline Fe II & 5362.87 & -2.74 & 23 & $3 d^{6}\left({ }^{3} \mathrm{G}\right) 4 s^{4} \mathrm{G}-3 d^{6}\left({ }^{5} \mathrm{D}\right) 4 p^{4} \mathrm{D}^{\circ}$ & 3.20 \\
\hline Fe II & 5414.07 & -3.79 & 24 & $3 d^{6}\left({ }^{3} \mathrm{G}\right) 4 s^{4} \mathrm{G}-3 d^{6}\left({ }^{5} \mathrm{D}\right) 4 p^{4} \mathrm{D}^{\circ}$ & 3.22 \\
\hline Fe II & 5425.26 & -3.36 & 24 & $3 d^{6}\left({ }^{3} \mathrm{G}\right) 4 s^{4} \mathrm{G}-3 d^{6}\left({ }^{5} \mathrm{D}\right) 4 p{ }^{4} \mathrm{~F}^{\circ}$ & 3.20 \\
\hline Fe II & 5432.97 & -3.63 & 24 & $3 d^{6}\left({ }^{3} \mathrm{H}\right) 4 s^{2} \mathrm{H}-3 d^{6}\left({ }^{5} \mathrm{D}\right) 4 p^{4} \mathrm{~F}^{\circ}$ & 3.27 \\
\hline Sc II & 5526.79 & 0.13 & 25 & $3 d^{2}{ }^{1} \mathrm{G}-3 d 4 p{ }^{1} \mathrm{~F}^{\circ}$ & 1.77 \\
\hline Fe II & 5534.85 & -2.93 & 25 & $3 d^{6}\left({ }^{3} \mathrm{H}\right) 4 s{ }^{2} \mathrm{H}-3 d^{6}\left({ }^{5} \mathrm{D}\right) 4 p^{4} \mathrm{~F}^{\circ}$ & 3.24 \\
\hline Sc II & 5641.00 & -1.04 & 26 & $3 d^{2}{ }^{3} \mathrm{P}-3 d 4 p^{3} \mathrm{P}^{\circ}$ & 1.50 \\
\hline Sc II & 5657.90 & -0.50 & 26 & $3 d^{2}{ }^{3} \mathrm{P}-3 d 4 p^{3} \mathrm{P}^{\circ}$ & 1.51 \\
\hline Sc II & 5658.36 & -1.17 & 26 & $3 d^{2}{ }^{3} \mathrm{P}-3 d 4 p^{3} \mathrm{P}^{\circ}$ & 1.50 \\
\hline Sc II & 5667.15 & -1.24 & 26 & $3 d^{2}{ }^{3} \mathrm{P}-3 d 4 p^{3} \mathrm{P}^{\circ}$ & 1.50 \\
\hline Sc II & 5669.04 & -1.12 & 26 & $3 d^{2}{ }^{3} \mathrm{P}-3 d 4 p^{3} \mathrm{P}^{\circ}$ & 1.50 \\
\hline Sc II & 5684.20 & -1.05 & 26 & $3 d^{2}{ }^{3} \mathrm{P}-3 d 4 p^{3} \mathrm{P}^{\circ}$ & 1.51 \\
\hline $\mathrm{Ba}$ II & 5853.67 & -1.00 & 29 & $5 d^{2} \mathrm{D}-6 p^{2} \mathrm{P}$ & 0.60 \\
\hline $\mathrm{He} \mathrm{I}^{\mathrm{e}}$ & 5875.63 & 0.74 & 28,29 & $1 s 2 p^{3} \mathrm{P}^{\circ}-1 s 3 d^{3} \mathrm{D}$ & 20.97 \\
\hline $\mathrm{Na} \mathrm{I}$ & 5889.95 & 0.12 & 29,27 & $3 s^{2} \mathrm{~S}-3 p^{2} \mathrm{P}^{\circ}$ & 0.00 \\
\hline $\mathrm{Na} \mathrm{I}$ & 5895.92 & -0.18 & 29,27 & $3 s^{2} \mathrm{~S}-3 p^{2} \mathrm{P}^{\circ}$ & 0.00 \\
\hline $\mathrm{Ba}$ II & 6141.71 & -0.08 & 30 & $5 d^{2} \mathrm{D}-6 p^{2} \mathrm{P}^{\circ}$ & 0.70 \\
\hline Fe II & 6147.74 & -2.72 & 30 & $3 d^{6}\left({ }^{3} \mathrm{D}\right) 4 s^{4} \mathrm{D}-3 d^{6}\left({ }^{5} \mathrm{D}\right) 4 p^{4} \mathrm{P}^{\circ}$ & 3.89 \\
\hline Fe II & 6149.26 & -2.72 & 30 & $3 d^{6}\left({ }^{3} \mathrm{D}\right) 4 s^{4} \mathrm{D}-3 d^{6}\left({ }^{5} \mathrm{D}\right) 4 p^{4} \mathrm{P}^{\circ}$ & 3.89 \\
\hline Fe II & 6238.39 & -2.63 & 31 & $3 d^{6}\left({ }^{3} \mathrm{D}\right) 4 s^{4} \mathrm{D}-3 d^{6}\left({ }^{5} \mathrm{D}\right) 4 p^{4} \mathrm{P}^{\circ}$ & 3.89 \\
\hline Sc II & 6245.64 & -0.98 & 31 & $3 d^{2}{ }^{3} \mathrm{P}-3 d 4 p^{3} \mathrm{D}^{\circ}$ & 1.51 \\
\hline Fe II & 6247.56 & -2.33 & 31 & $3 d^{6}\left({ }^{3} \mathrm{D}\right) 4 s{ }^{4} \mathrm{D}-3 d^{6}\left({ }^{5} \mathrm{D}\right) 4 p^{4} \mathrm{P}^{\circ}$ & 3.89 \\
\hline Sc II & 6279.75 & -1.21 & 31 & $3 d^{2}{ }^{3} \mathrm{P}-3 d 4 p^{3} \mathrm{D}^{\circ}$ & 1.50 \\
\hline Si II & 6347.11 & 0.30 & 32 & $3 s^{2}\left({ }^{1} \mathrm{~S}\right) 4 s^{2} \mathrm{~S}-3 s^{2}\left({ }^{1} \mathrm{~S}\right) 4 p^{2} \mathrm{P}^{\circ}$ & 8.12 \\
\hline Si II & 6371.37 & -0.00 & 32 & $3 s^{2}\left({ }^{1} \mathrm{~S}\right) 4 s^{2} \mathrm{~S}-3 s^{2}\left({ }^{1} \mathrm{~S}\right) 4 p{ }^{2} \mathrm{P}^{\circ}$ & 8.12 \\
\hline H I & 6562.80 & 0.71 & 34,33 & $\ldots d$ & 10.20 \\
\hline
\end{tabular}


Table 4. SN II-P Photospheric-Phase Absorption Line Identifications - Continued

\begin{tabular}{lccccc}
\hline \hline Ion & Wavelength $(\AA)^{\mathrm{a}}$ & $\log (g f)$ & Feature $\mathrm{ID}^{\mathrm{b}}$ & Designation & $E(\mathrm{eV})^{\mathrm{c}}$ \\
\hline Ca II & 8498.02 & -1.31 & 35 & $3 d^{2} \mathrm{D}-4 p^{2} \mathrm{P}^{\circ}$ & 1.69 \\
Ca II & 8662.14 & -0.62 & 36 & $3 d^{2} \mathrm{D}-4 p^{2} \mathrm{P}^{\circ}$ & 1.69 \\
\hline \multicolumn{5}{c}{$\mathrm{i}$}
\end{tabular}

Note. - Lines producing or contributing to absorption features in the optical spectrum of SN 1999em during the photospheric phase. Rest wavelengths, oscillators strengths, configurations, terms, and energies taken from the Kurucz (1996) spectral line database.

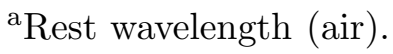

${ }^{\mathrm{b}}$ Feature number(s) corresponding to the identifications shown in Figures 9 and 10.

${ }^{\mathrm{c}}$ Energy of the lower energy state of the transition.

${ }^{\mathrm{d}}$ For H I, the contributing closely-spaced configurations and terms are: $2 p{ }^{2} \mathrm{P}^{\circ}-\mathrm{n} d{ }^{2} D, 2 s 2 \mathrm{~S}-$ $\mathrm{n} p{ }^{2} \mathrm{P}^{\circ}$, and $2 p{ }^{2} \mathrm{P}^{\circ}-\mathrm{n} s{ }^{2} \mathrm{~S}$, where $\mathrm{n}$ is the principle quantum number of the upper state of the transition (i.e., 3 for $\mathrm{H} \alpha, 4$ for $\mathrm{H} \beta$, etc.).

${ }^{\text {e}}$ For brevity, we do not list the 6 closely-spaced components of the He I $\lambda 5876$ multiplet, but rather list the mean wavelength and total oscillator strength of the line. 


\subsubsection{Line Features Associated with P-Cygni Profiles}

In all, there are five features (labeled 10, 14, 15, 27, and 33 in Figure 10) for which no convincing line ID is found near the rest wavelength inferred by assuming a velocity similar to that derived from the more readily identified lines. All five features appear at multiple epochs and follow the general redward drift with time seen in the other lines. We propose that three of these features $(14$, 27 , and 33) are actually associated with the strong P-Cygni absorptions of $\mathrm{H} \beta \lambda 4861$, Na I D $\lambda 5892$, and $\mathrm{H} \alpha \lambda 6563$, respectively, and represent the continued evolution of the low-contrast features first identified in the spectrum from day 7 (these features can be seen as small "notches" just blueward of the $\mathrm{H} \beta$ and $H \gamma$ absorption in Figure 9; due to contamination from telluric features, the "notch" is not positively confirmed in $\mathrm{H} \alpha$ on day 7 - see L01). From day 30 until the end of the plateau these features mirror the other lines' decreasing velocity (about 2,000 $\mathrm{km} \mathrm{s}^{-1}$ during this period of time), but with a velocity about $8000 \mathrm{~km} \mathrm{~s}^{-1}$ higher. It is possible that the other two unidentified features (10 and 15) are also high-velocity absorptions associated with strong lines: feature 10 with the large metal blend of Ba II, Ti II, and Fe II near $\lambda 4554$ (the candidate producing absorption most consistent with the other high-velocity features is Fe II $\lambda 4584$ ), and feature 15 with Ba II $\lambda 4934$. These identifications are less secure, however, given the relative weakness of the responsible lines. We note that Fassia et al. (1998) identify feature 33 with Sc II $\lambda 6378$. While this ID does yield a velocity similar to other photospheric lines, the feature is evident in neither the simulated spectra of Hatano et al. (1999) nor the synthetic spectrum of Weaver \& Woosley (1993) that we analyzed. The Kurucz (1996) line list does confirm a relatively strong Sc II feature at $\lambda 6378.7$, but it lies $7.4 \mathrm{eV}$ above the ground state. For it to be visible, many other features of similar strength and excitation should be observed as well, and they are not. We thus favor the identification of this feature with $\mathrm{H} \alpha$.

Figure 12 shows the development of the $\mathrm{H} \alpha$ profile from day 40 until our latest observation on day 517. In addition to the high-velocity absorption feature already discussed (labeled $A$ in Figure 12), a curious "absorption line," labeled $B$, develops between day 49 and day 76 and disappears by day 124 . If associated with $\mathrm{H} \alpha$, the corresponding velocity is quite similar to the velocity derived from weaker lines, such as Fe II $\lambda$ 5018. A similar feature was also observed in the Type II-P SN 1999gi (Leonard \& Filippenko 2001), as well as in SN 1988A (Turatto et al. 1993), and perhaps SN 1985L (Filippenko \& Sargent 1986). A likely explanation is that this feature is produced by Chugai's (1991; see also Utrobin, Chugai, \& Andronova 1995) proposed mechanism for the anomalous blueshifted "absorption" seen in SN 1987A (i.e., the "Bochum event"; see also Phillips \& Heathcote 1989). Under this model, the feature is actually produced by a lack of absorption by gas at velocities surrounding the "dip," caused by ineffective screening of continuum photons due to excitation stratification in the envelope.

Finally, there is the development of a distinct "notch" in the $\mathrm{H} \alpha, \mathrm{H} \beta$, and [Ca II] $\lambda \lambda 7291,7324$

profiles near their rest wavelengths, which develops immediately after the end of the plateau (Figure 12). As discussed by L01, this feature might originate within the expanding ejecta itself, perhaps due to an asymmetry in the line-emitting region. It is possible that it is produced by the 


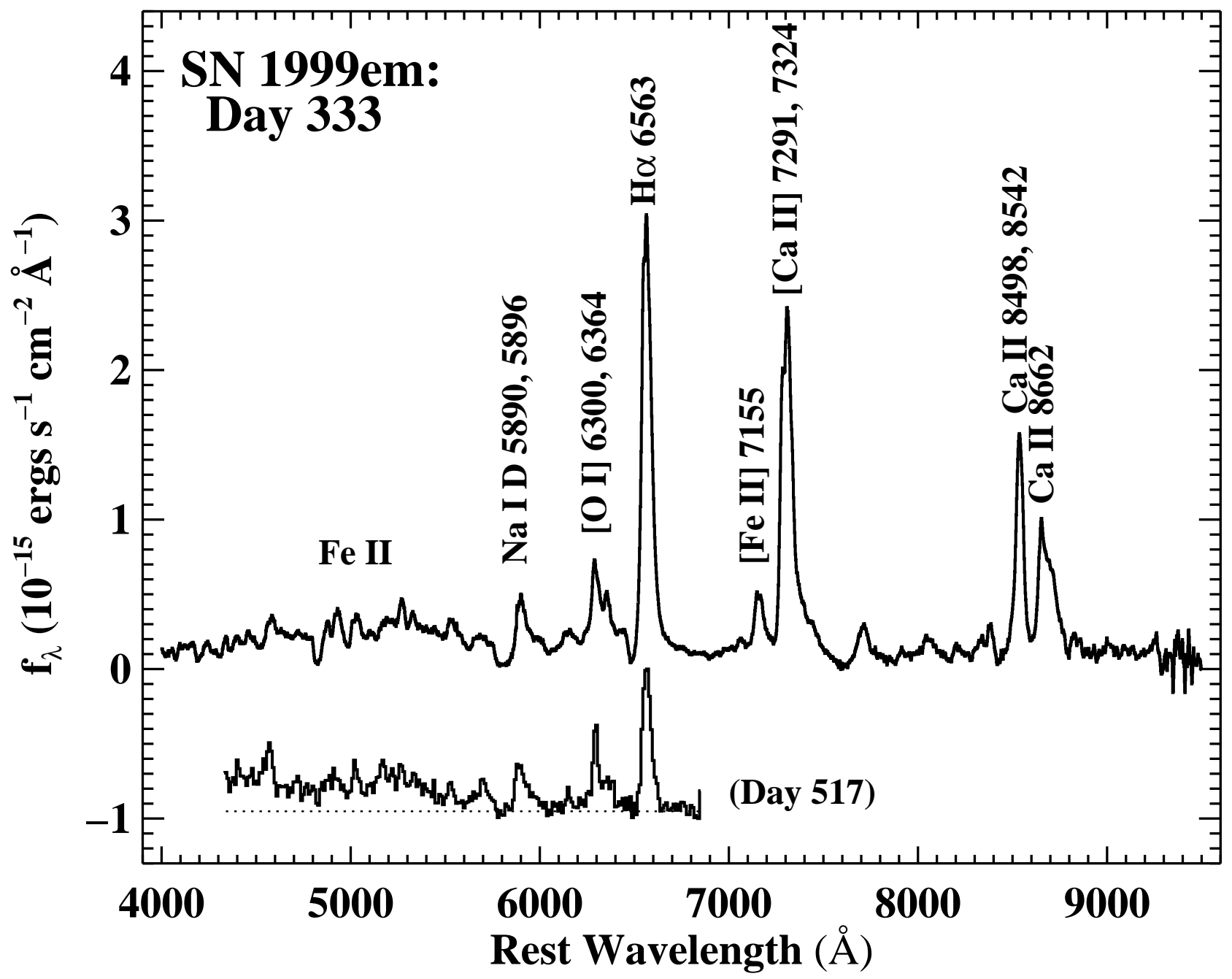

Fig. 11. - SN 1999em in the nebular phase, 333 days after discovery, with prominent emission lines identified; redward of $\lambda 7780$ the displayed spectrum is from day 313. For comparison of features, the spectrum from day 517 is also shown, having been scaled by a factor of 12.6 and then offset by -0.95; the zero-flux level is indicated (dotted line). 


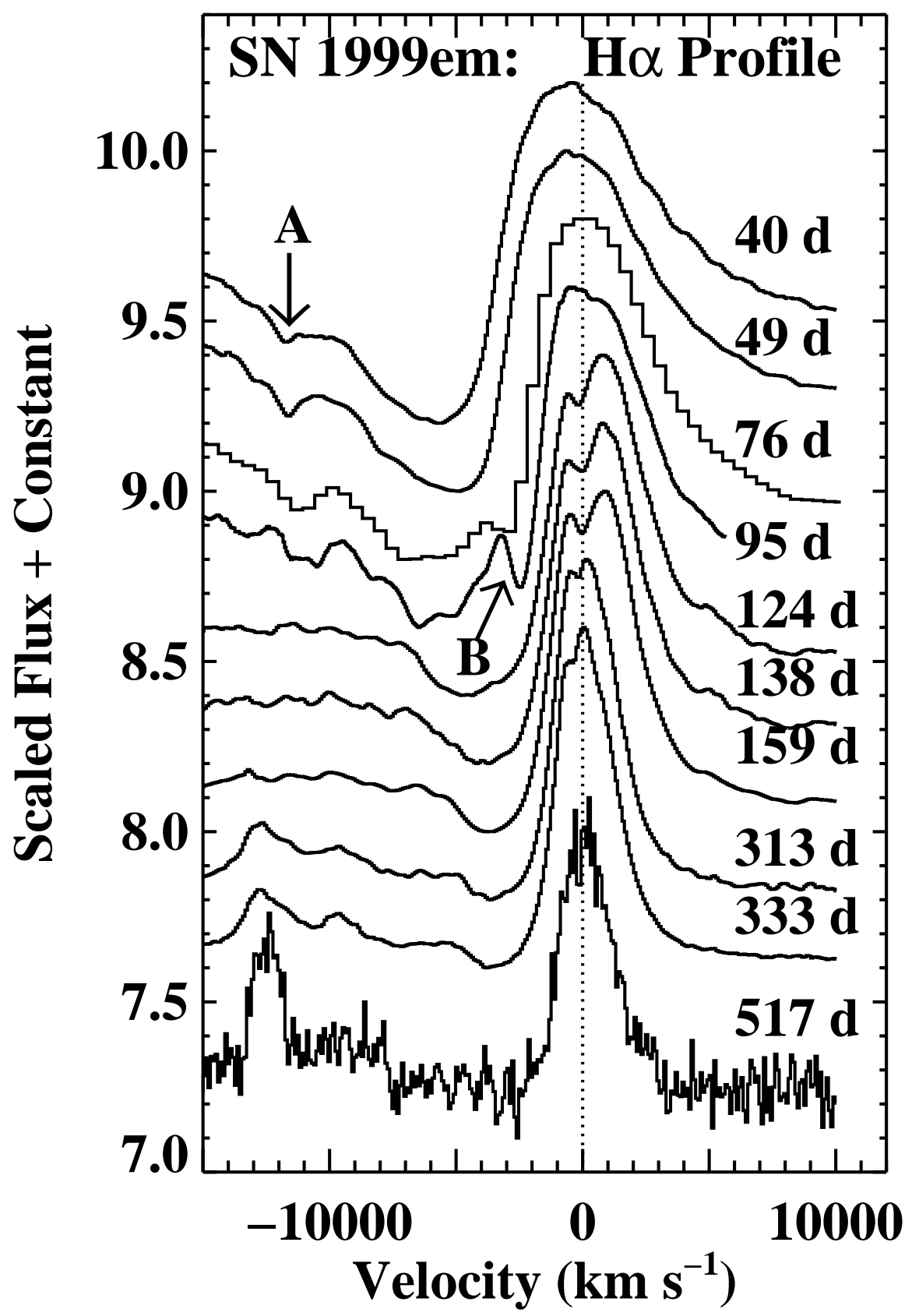

Fig. 12. - The development of the $\mathrm{H} \alpha$ profile of SN 1999em, with days since discovery (1999-11-29 UT) indicated. Of particular interest are the "notch" near zero velocity that is first seen on day 124 (perhaps a hint on day 95), a "dip" $(B)$ on the blue side of the profile on days 76 and 95, and a moderately strong absorption feature $(A)$ at high velocity. The notch near zero velocity is seen in other strong emission lines (see L01), and the high-velocity absorption feature $(A)$ is seen in the $\mathrm{H} \beta$ and Na I $\lambda 5892$ profiles as well. The plateau phase ended about 95 days after discovery, the same period during which features $A$ and $B$ disappear and the notch at zero velocity is first seen. Note that the spectrum from day 76 has very low resolution. 
ejecta interacting with circumstellar material, although the lack of any other evidence suggesting significant circumstellar interaction (e.g., Pooley et al., 2001) makes us doubt this possibility. The notch seems to diminish in strength with time, and it may not be present on day 517, although the low signal-to-noise ratio $(\mathrm{S} / \mathrm{N})$ of that spectrum renders it insensitive to low-contrast features. It is also interesting to note the many other features that complicate the $\mathrm{H} \alpha$ absorption profile as SN 1999 em makes the transition out of the plateau epoch, particularly evident in the day 95 spectrum; these features are also seen in a spectrum of SN 1999gi at a similar epoch; in fact, the exact correspondence of all of the line featurs between these two SNe II-P is quite remarkable (Leonard \& Filippenko 2001).

\subsubsection{Inferring Photospheric Velocity from Absorption Lines}

From the lines identified in Figure 10, we find four weak, unblended spectral features that should serve as good photospheric velocity indicators to compare with the velocity determined by the stronger Fe II $\lambda \lambda 4924,5018,5169$ lines: Fe II $\lambda 4629$ (feature 12), Sc II $\lambda 4670$ (feature 13), Fe II $\lambda 5276$ (feature 21), and Fe II $\lambda 5318$ (feature 22). To translate the measured wavelength minima into velocities we must remove the redshift of SN 1999em and assign rest wavelengths to all of the line features. The NASA/IPAC Extragalactic Database (NED) recession velocity of NGC 1637 is $717 \mathrm{~km} \mathrm{~s}^{-1}$. However, since the host galaxy is somewhat inclined $\left(i=32^{\circ}\right)$ and the location of the SN within the spiral arm is not known, the NED velocity may differ significantly from the actual velocity of the SN. One way sometimes used to infer the recession velocity of a SN is to measure the velocity of bright H II regions on both sides of the SN along the slit, and interpolate to the position of the SN. When this is done for SN 1999em on the frames that contain bright H II regions, however, values from 690 to $900 \mathrm{~km} \mathrm{~s}^{-1}$ result; in other words, we find the inferred velocity to be a function of which H II regions fall along the slit, which is determined by the slit's position angle. As shown in Figure 14, the Na I D interstellar absorption from NGC 1637 occurs over a range from about 700 to $820 \mathrm{~km} \mathrm{~s}^{-1}$. We are thus left with some uncertainty in the exact recession velocity of SN 1999em. This uncertainty ultimately leads to a small systematic uncertainty in our derived

EPM distance to SN 1999em. For our analysis, we assign a recession velocity to SN $1999 \mathrm{em}$ of 800 $\mathrm{km} \mathrm{s}^{-1}$ and a $1 \sigma$ uncertainty of $50 \mathrm{~km} \mathrm{~s}^{-1}$.

Assigning a single rest wavelength to each of the features identified in Figures 9 and 10 is complicated by the blending that exists in many of the lines, and simply taking the rest wavelength of the dominant line may lead to inaccuracies. When a feature consists of a closely spaced multiplet of a single ion, we estimate the rest wavelength as

$$
\lambda_{\circ}=\frac{\sum_{i=1}^{n} \lambda_{i} \times(g f)_{i}}{\sum_{i=1}^{n}(g f)_{i}},
$$

where $i$ is the line number and $(g f)$ is the oscillator strength. Since it has been previously used as a photospheric velocity indicator (e.g., Schmidt et al. 1994a), we note that the 6 lines of the 
multiplet of Sc II comprising feature 26 yield $\lambda_{\circ}=5665.6 \AA$, which is significantly different from the rest wavelength of the strongest component, $\lambda_{\circ}=5657.9 \AA$; using $\lambda_{\circ}=5657.9 \AA$, as was evidently done in previous studies, would therefore result in derived velocities that are $\sim 400 \mathrm{~km} \mathrm{~s}^{-1}$ lower than is appropriate for this feature. For features that contain blends of lines from different ions (or different energy levels within the same ion), the assigned rest wavelength was guided by estimates of the relative strengths of the individual lines during the photospheric phase. In many cases, though (i.e., Fe II $\lambda 4924$ and Sc II $\lambda 5527$ ), the relative strengths of the individual lines likely vary considerably, diminishing their reliability as simple photospheric velocity indicators.

To assign an uncertainty to the inferred velocities, we attempt to quantify the error resulting from uncertainty in both the wavelength scale and the measurement process itself. To gauge the uncertainty in our wavelength scale, we cross-correlated the region around the strong $\mathrm{Na} \mathrm{I} \mathrm{D}$ $\lambda \lambda 5890,5896$ host galaxy interstellar absorption line in every low-resolution spectrum with this region in our day 95 spectrum, which has an excellent $\mathrm{S} / \mathrm{N}$. The $1 \sigma$ spread of wavelength offsets was found to be $0.6 \AA$, which we adopt as the uncertainty in our derived wavelength scale for every spectrum.

To measure the wavelength of maximum absorption and derive its measurement uncertainty, we first estimated the local "continuum" shape by fitting a straight line to points near the two local flux peaks on either side of the absorption feature. We then normalized the absorption feature by dividing it by the interpolated continuum fit. To estimate the wavelength of minimum flux in the normalized line profile, we then fit a series of third-order Savitsky-Golay smoothing polynomials (Press et al. 1992) to the data with widths ranging from $1 / 2$ to $3 / 2$ the FWHM of the line profile and then averaged the fits; this was found to produce more robust results than using a single smoothing function alone. The minimum of the smoothed spectrum was then found, interpolated to the nearest $0.01 \AA$. To estimate the measurement error, we added random noise at the level seen in the data to the smoothed spectrum and used an automatic routine to find the absorption minimum in 1000 sets of noisy artificial data. The standard deviation of these measurements added in quadrature to the $0.6 \AA$ uncertainty in our wavelength scale is the final uncertainty reported for the line's absorption minimum. We do not attempt to quantify the systematic uncertainty in a feature's assigned rest wavelength, but caution that for some blends this systematic error may dominate the total (statistical and systematic) uncertainty.

We characterize each feature's strength by measuring its "depth," defined as

$$
d=\left(f_{\mathrm{c}}-f_{\min }\right) / f_{c},
$$

where $f_{\min }$ is the flux at the line's minimum and $f_{c}$ is the value of the interpolated continuum flux at the location of the line's minimum. The measured depth in some cases depends significantly on the strength of adjacent features since this affects the location of the points used to fit the normalizing continuum. In general, though, $d$ should be a reasonably good indicator of line strength. The rest wavelengths adopted for all features and the measured velocities, velocity uncertainties, and line depths are given in Table 5. 
Table 5. SN 1999em: Absorption Line Measurements

\begin{tabular}{|c|c|c|c|c|c|c|c|c|c|c|c|}
\hline \multirow[b]{2}{*}{ Line $^{b}$} & \multirow[b]{2}{*}{ Ion $^{\mathrm{c}}$} & \multirow[b]{2}{*}{ Wavelength $(\AA)$} & \multicolumn{2}{|c|}{ Day $2^{\mathrm{a}}$} & \multicolumn{2}{|c|}{ Day 3} & \multicolumn{2}{|c|}{ Day 4} & \multicolumn{3}{|c|}{ Day 5} \\
\hline & & & Vel. & $\operatorname{Depth}^{\mathrm{f}}$ & Vel. & Depth & Vel. & Depth & Vel. & Depth & \\
\hline 1 & $\mathrm{H} \zeta$ & 3889.05 & $\ldots$ & $\ldots$ & $\ldots$ & $\ldots$ & $\ldots$ & $\ldots$ & $\ldots$ & $\ldots$ & \\
\hline 2 & $\mathrm{H} \epsilon$ & 3970.07 & $\ldots$ & $\ldots$ & $\ldots$ & $\ldots$ & $\ldots$ & $\ldots$ & $\ldots$ & $\ldots$ & \\
\hline 3 & Sr II: & 4077.71 & $\ldots$ & $\ldots$ & $\ldots$ & $\ldots$ & $\ldots$ & $\ldots$ & $\ldots$ & $\ldots$ & \\
\hline 4 & $\mathrm{H} \delta$ & 4101.77 & $\cdots$ & $\cdots$ & $\cdots$ & $\cdots$ & $\cdots$ & $\cdots$ & $\cdots$ & $\cdots$ & \\
\hline 5 & Fe II: & 4180.98 & $\ldots$ & $\cdots$ & $\cdots$ & $\ldots$ & $\cdots$ & $\ldots$ & $\cdots$ & $\ldots$ & \\
\hline 6 & Sc II: & 4241.00 & $\cdots$ & $\ldots$ & $\ldots$ & $\ldots$ & $\ldots$ & $\ldots$ & $\ldots$ & $\ldots$ & \\
\hline 7 & Fe II: & 4301.70 & $\ldots$ & $\ldots$ & $\ldots$ & $\ldots$ & $\ldots$ & $\ldots$ & $\ldots$ & $\ldots$ & \\
\hline 8 & $\mathrm{H} \gamma$ & 4340.49 & $9020(240)$ & 0.08 & $8710(560)$ & 0.10 & $8500(340)$ & 0.12 & $8340(180)$ & 0.18 & \\
\hline 9 & Sc II: & 4393.00 & $\ldots$ & $\ldots$ & $\ldots$ & $\ldots$ & $\ldots$ & $\ldots$ & $\ldots$ & $\ldots$ & \\
\hline 10 & No ID & $\ldots$ & $\ldots$ & $\ldots$ & $\ldots$ & $\ldots$ & $\ldots$ & $\ldots$ & $\ldots$ & $\ldots$ & \\
\hline 11 & Ba II: & 4554.03 & $\ldots$ & $\ldots$ & $\ldots$ & $\ldots$ & $\ldots$ & $\ldots$ & $\ldots$ & $\ldots$ & \\
\hline 12 & Fe II & 4629.34 & $\ldots$ & $\ldots$ & $\ldots$ & $\ldots$ & $\ldots$ & $\ldots$ & $\ldots$ & $\ldots$ & \\
\hline 13 & Sc II & 4670.41 & $\ldots$ & $\ldots$ & $\ldots$ & $\ldots$ & $\ldots$ & $\ldots$ & $\ldots$ & $\ldots$ & 1 \\
\hline 14 & $\mathrm{H} \beta \mathrm{PCyg}$ & 4861.36 & $\ldots$ & $\ldots$ & $\ldots$ & $\ldots$ & $\ldots$ & $\ldots$ & $\ldots$ & $\ldots$ & co \\
\hline 15 & No ID & $\ldots$ & $\ldots$ & $\ldots$ & $\ldots$ & $\ldots$ & $\ldots$ & $\ldots$ & $\ldots$ & $\ldots$ & $\infty$ \\
\hline 16 & $\mathrm{H} \beta$ & 4861.36 & $10460(360)$ & 0.22 & $10080(110)$ & 0.27 & $10040(120)$ & 0.29 & $9410(90)$ & 0.33 & 1 \\
\hline 17 & $\mathrm{Fe} \mathrm{II}^{\mathrm{g}}$ & 4923.93 & $\ldots$ & $\ldots$ & $\ldots$ & $\ldots$ & $\ldots$ & $\ldots$ & $\ldots$ & $\ldots$ & \\
\hline 18 & Fe II & 5018.44 & $\ldots$ & $\ldots$ & $\ldots$ & $\ldots$ & $\ldots$ & $\ldots$ & $\ldots$ & $\ldots$ & \\
\hline 19 & Fe II & 5169.03 & $\ldots$ & $\ldots$ & $\ldots$ & $\ldots$ & $\ldots$ & $\ldots$ & $\ldots$ & $\ldots$ & \\
\hline 20 & Fe II: & 5234.63 & $\ldots$ & $\ldots$ & $\ldots$ & $\ldots$ & $\ldots$ & $\ldots$ & $\ldots$ & $\ldots$ & \\
\hline 21 & Fe II & 5276.00 & $\cdots$ & $\cdots$ & $\cdots$ & $\ldots$ & $\ldots$ & $\ldots$ & $\ldots$ & $\ldots$ & \\
\hline 22 & Fe II & 5318.00 & $\ldots$ & $\ldots$ & $\ldots$ & $\ldots$ & $\ldots$ & $\ldots$ & $\ldots$ & $\ldots$ & \\
\hline 23 & Fe II & 5362.87 & $\ldots$ & $\ldots$ & $\ldots$ & $\ldots$ & $\cdots$ & $\ldots$ & $\ldots$ & $\ldots$ & \\
\hline 24 & Fe II: & 5425.00 & $\ldots$ & $\ldots$ & $\ldots$ & $\ldots$ & $\ldots$ & $\ldots$ & $\ldots$ & $\ldots$ & \\
\hline 25 & Sc II: & 5533.00 & $\ldots$ & $\ldots$ & $\ldots$ & $\ldots$ & $\ldots$ & $\ldots$ & $\ldots$ & $\ldots$ & \\
\hline 26 & Sc II: & 5665.60 & $\ldots$ & $\ldots$ & $\ldots$ & $\ldots$ & $\ldots$ & $\ldots$ & $\ldots$ & $\ldots$ & \\
\hline 27 & Na I PCyg & 5892.00 & $\ldots$ & $\ldots$ & $\ldots$ & $\ldots$ & $\ldots$ & $\ldots$ & $\ldots$ & $\ldots$ & \\
\hline 28 & $\mathrm{He} \mathrm{I}^{\mathrm{h}}$ & 5875.63 & $9330(120)$ & 0.09 & $9240(120)$ & 0.10 & $8580(70)$ & 0.13 & $8300(80)$ & 0.10 & \\
\hline 29 & Na ID: ${ }^{i}$ & 5892.00 & $\ldots$ & $\ldots$ & $\ldots$ & $\ldots$ & $\ldots$ & $\ldots$ & $\ldots$ & $\ldots$ & \\
\hline 30 & Ba II: & 6147.00 & $\ldots$ & $\ldots$ & $\ldots$ & $\ldots$ & $\ldots$ & $\ldots$ & $\ldots$ & $\ldots$ & \\
\hline 31 & Sc II: & 6254.00 & $\ldots$ & $\ldots$ & $\ldots$ & $\ldots$ & $\ldots$ & $\ldots$ & $\ldots$ & $\ldots$ & \\
\hline 32 & Si II & 6355.20 & $\ldots$ & $\ldots$ & $\ldots$ & $\ldots$ & $\ldots$ & $\ldots$ & $\ldots$ & $\ldots$ & \\
\hline 33 & $\mathrm{H} \alpha \mathrm{PCyg}$ & 6562.85 & $\ldots$ & $\ldots$ & $\ldots$ & $\ldots$ & $\ldots$ & $\ldots$ & $\ldots$ & $\ldots$ & \\
\hline 34 & $\mathrm{H} \alpha$ & 6562.85 & $10810(400)$ & 0.27 & $10540(70)$ & 0.32 & $10210(70)$ & 0.35 & $9560(110)$ & 0.34 & \\
\hline 35 & $\mathrm{Ca}$ II & 8498.02 & $\ldots$ & $\ldots$ & $\ldots$ & $\ldots$ & $\ldots$ & $\ldots$ & $\ldots$ & $\ldots$ & \\
\hline 36 & $\mathrm{Ca}$ II & 8662.14 & $\ldots$ & $\ldots$ & $\ldots$ & $\cdots$ & $\cdots$ & $\cdots$ & $\cdots$ & $\ldots$ & \\
\hline
\end{tabular}


Table 5 - Continued.

\begin{tabular}{|c|c|c|c|c|c|c|c|c|c|c|}
\hline \multirow[b]{2}{*}{ Line } & \multicolumn{2}{|c|}{ Day 6} & \multicolumn{2}{|c|}{\begin{tabular}{l|l} 
Day 7 \\
\end{tabular}} & \multicolumn{2}{|c|}{ Day 8} & \multicolumn{2}{|c|}{ Day 9} & \multicolumn{2}{|c|}{ Day 10} \\
\hline & Vel. & Depth & Vel. & Depth & Vel. & Depth & Vel. & Depth & Vel. & Depth \\
\hline 1 & $\ldots$ & $\ldots$ & $7270(80)$ & 0.04 & $\cdots$ & $\cdots$ & $\ldots$ & $\cdots$ & $\ldots$ & $\ldots$ \\
\hline 2 & $\ldots$ & $\ldots$ & $7320(180)$ & 0.05 & $\ldots$ & $\ldots$ & $\ldots$ & $\ldots$ & $\ldots$ & $\ldots$ \\
\hline 3 & $\ldots$ & $\ldots$ & $\ldots$ & $\ldots$ & $\ldots$ & $\ldots$ & $\ldots$ & $\ldots$ & $\ldots$ & $\ldots$ \\
\hline 4 & $\ldots$ & $\ldots$ & $7450(40)$ & 0.11 & $6580(200)$ & $\ldots$ & $6210(690)$ & $\ldots$ & $\ldots$ & $\ldots$ \\
\hline 5 & $\ldots$ & $\ldots$ & $\ldots$ & $\ldots$ & $\ldots$ & $\ldots$ & $\ldots$ & $\ldots$ & $\ldots$ & $\ldots$ \\
\hline 6 & $\ldots$ & $\ldots$ & $\ldots$ & $\ldots$ & $\ldots$ & $\ldots$ & $\ldots$ & $\ldots$ & $\ldots$ & $\ldots$ \\
\hline 7 & $\ldots$ & $\ldots$ & $\ldots$ & $\ldots$ & $\ldots$ & $\ldots$ & $\ldots$ & $\ldots$ & $\ldots$ & $\ldots$ \\
\hline 8 & $8350(120)$ & 0.16 & $7990(70)$ & 0.26 & $7910(110)$ & 0.24 & $7580(100)$ & 0.26 & $7290(80)$ & 0.23 \\
\hline 9 & $\ldots$ & $\ldots$ & $\ldots$ & $\ldots$ & $\ldots$ & $\ldots$ & $\ldots$ & $\ldots$ & $\ldots$ & $\ldots$ \\
\hline 10 & $\ldots$ & $\ldots$ & $\ldots$ & $\ldots$ & $\ldots$ & $\ldots$ & $\ldots$ & $\ldots$ & $\ldots$ & $\ldots$ \\
\hline 11 & $\ldots$ & $\ldots$ & $\ldots$ & $\ldots$ & $\ldots$ & $\ldots$ & $\ldots$ & $\ldots$ & $9380(180)$ & 0.19 \\
\hline 12 & $\ldots$ & $\ldots$ & $\ldots$ & $\ldots$ & $\ldots$ & $\ldots$ & $\ldots$ & $\ldots$ & $\ldots$ & $\ldots$ \\
\hline 13 & $\ldots$ & $\ldots$ & $\ldots$ & $\ldots$ & $\ldots$ & $\ldots$ & $\ldots$ & $\ldots$ & $\ldots$ & $\ldots$ \\
\hline 14 & $\ldots$ & $\ldots$ & $12780(60)$ & 0.02 & $\ldots$ & $\ldots$ & $\ldots$ & $\ldots$ & $\ldots$ & $\ldots$ \\
\hline 15 & $\ldots$ & $\ldots$ & $\ldots$ & $\cdots$ & $\ldots$ & $\cdots$ & $\ldots$ & $\cdots$ & $\ldots$ & $\cdots$ \\
\hline 16 & $9000(100)$ & 0.29 & $8640(40)$ & 0.37 & $8570(60)$ & 0.38 & $8480(60)$ & 0.38 & $7920(60)$ & 0.39 \\
\hline 17 & $\ldots$ & $\ldots$ & .. & $\ldots$ & ... & $\ldots$ & - & & & \\
\hline 18 & $\ldots$ & $\ldots$ & $\ldots$ & $\ldots$ & $\ldots$ & $\ldots$ & $7180(570)$ & 0.03 & $7030(200)$ & 0.06 \\
\hline 19 & $\cdots$ & $\cdots$ & $8030(130)$ & 0.02 & $8080(280)$ & 0.07 & $7690(180)$ & 0.09 & $7260(100)$ & 0.15 \\
\hline 20 & $\cdots$ & $\cdots$ & $\ldots$ & $\ldots$ & $\ldots$ & $\cdots$ & $\ldots$ & $\ldots$ & $\ldots$ & $\ldots$ \\
\hline 21 & $\cdots$ & $\cdots$ & $\cdots$ & $\cdots$ & $\cdots$ & $\cdots$ & $\cdots$ & $\cdots$ & $\cdots$ & $\cdots$ \\
\hline 22 & $\cdots$ & $\cdots$ & $\cdots$ & $\cdots$ & $\cdots$ & $\cdots$ & $\cdots$ & $\cdots$ & $\cdots$ & $\cdots$ \\
\hline 23 & $\cdots$ & $\cdots$ & $\cdots$ & $\cdots$ & $\cdots$ & $\cdots$ & $\cdots$ & $\cdots$ & $\cdots$ & $\cdots$ \\
\hline 24 & $\cdots$ & $\cdots$ & $\cdots$ & $\cdots$ & $\cdots$ & $\cdots$ & $\cdots$ & $\cdots$ & $\cdots$ & $\cdots$ \\
\hline 25 & $\cdots$ & $\cdots$ & $\cdots$ & $\cdots$ & $\cdots$ & $\cdots$ & $\cdots$ & $\cdots$ & $\cdots$ & $\cdots$ \\
\hline 26 & $\begin{array}{l}\cdots \\
\ldots\end{array}$ & $\begin{array}{l}\cdots \\
\ldots\end{array}$ & $\begin{array}{l}\cdots \\
\ldots\end{array}$ & $\begin{array}{l}\cdots \\
\ldots\end{array}$ & $\begin{array}{l}\cdots \\
\cdots\end{array}$ & $\begin{array}{l}\cdots \\
\ldots\end{array}$ & $\begin{array}{l}\cdots \\
\cdots\end{array}$ & $\begin{array}{l}\cdots \\
\ldots\end{array}$ & $\begin{array}{l}\cdots \\
\ldots\end{array}$ & $\begin{array}{l}\cdots \\
\cdots\end{array}$ \\
\hline $\begin{array}{l}27 \\
28\end{array}$ & $8110(280)$ & $\cdots$ & $7260(70)$ & 0.09 & $7070(220)$ & 0.07 & $6810(220)$ & 0.04 & $5810(90)$ & 0.04 \\
\hline 29 & $\ldots$ & $\ldots$ & $\ldots$ & $\ldots$ & $\ldots$ & $\ldots$ & $\ldots$ & $\ldots$ & ... & $\ldots$ \\
\hline 30 & $\ldots$ & $\ldots$ & $\ldots$ & $\ldots$ & $\ldots$ & $\ldots$ & $\ldots$ & $\ldots$ & $\ldots$ & $\ldots$ \\
\hline 31 & $\ldots$ & $\ldots$ & $\ldots$ & $\ldots$ & $\ldots$ & $\ldots$ & $\ldots$ & $\ldots$ & $\ldots$ & $\ldots$ \\
\hline 32 & $\cdots$ & $\ldots$ & $7890(120)$ & 0.03 & $\ldots$ & $\ldots$ & $\ldots$ & $\ldots$ & $6640(120)$ & 0.07 \\
\hline 33 & $\ldots$ & $\ldots$ & $\ldots$ & $\ldots$ & $\ldots$ & $\ldots$ & $\ldots$ & $\ldots$ & $\ldots$ & $\ldots$ \\
\hline 34 & $9550(160)$ & 0.34 & $9820(50)$ & 0.48 & $9560(60)$ & 0.43 & $9260(60)$ & 0.44 & $9010(40)$ & 0.51 \\
\hline 35 & $\cdots$ & $\cdots$ & $\cdots$ & $\cdots$ & $\cdots$ & $\cdots$ & $\cdots$ & $\cdots$ & $\cdots$ & $\cdots$ \\
\hline 36 & $\cdots$ & $\cdots$ & $\cdots$ & $\cdots$ & $\cdots$ & $\cdots$ & $\cdots$ & $\cdots$ & $\cdots$ & $\cdots$ \\
\hline
\end{tabular}


Table 5 - Continued.

\begin{tabular}{|c|c|c|c|c|c|c|c|c|c|c|}
\hline \multirow[b]{2}{*}{ Line } & \multicolumn{2}{|c|}{ Day 11} & \multicolumn{2}{|c|}{ Day 21} & \multicolumn{2}{|c|}{ Day 24} & \multicolumn{2}{|c|}{ Day 26} & \multicolumn{2}{|c|}{ Day 30} \\
\hline & Vel. & Depth & Vel. & Depth & Vel. & Depth & Vel. & Depth & Vel. & Depth \\
\hline 1 & $\cdots$ & $\cdots$ & $\cdots$ & $\cdots$ & $\cdots$ & $\cdots$ & $\cdots$ & $\cdots$ & $\cdots$ & $\cdots$ \\
\hline 2 & $\ldots$ & $\cdots$ & $\ldots$ & $\cdots$ & $\ldots$ & $\cdots$ & $\ldots$ & $\cdots$ & $\ldots$ & $\cdots$ \\
\hline 3 & $\cdots$ & $\cdots$ & $3670(220)$ & $\cdots$ & $3170(90)$ & $\cdots$ & $2890(90)$ & $\cdots$ & $3250(190)$ & $\cdots$ \\
\hline 4 & $\cdots$ & $\cdots$ & $\cdots$ & $\cdots$ & $\ldots$ & $\cdots$ & & $\cdots$ & & $\cdots$ \\
\hline 5 & $\cdots$ & $\cdots$ & $\cdots$ & $\cdots$ & $\ldots$ & $\cdots$ & $5190(80)$ & 0.24 & $4570(340)$ & 0.17 \\
\hline 6 & $\cdots$ & $\cdots$ & $\ldots$ & $\cdots$ & $5030(130)$ & 0.22 & $4920(240)$ & 0.17 & $4640(180)$ & 0.21 \\
\hline 7 & $\ldots$ & $\cdots$ & $4040(130)$ & 0.44 & $4040(160)$ & 0.39 & $3690(180)$ & 0.38 & $3550(170)$ & 0.39 \\
\hline 8 & $7150(150)$ & 0.29 & $\ldots$ & $\cdots$ & $\ldots$ & $\cdots$ & $\ldots$ & $\cdots$ & $\ldots$ & $\ldots$ \\
\hline 9 & $\ldots$ & $\ldots$ & $\ldots$ & $\ldots$ & $\ldots$ & $\ldots$ & $4840(250)$ & 0.11 & $\ldots$ & $\ldots$ \\
\hline 10 & $\ldots$ & $\ldots$ & $\ldots$ & $\ldots$ & $\cdots$ & $\cdots$ & {$[4383.7(6.9)]$} & 0.05 & $\cdots$ & $\cdots$ \\
\hline 11 & $8260(60)$ & 0.18 & $5390(110)$ & 0.45 & $\ldots$ & $\ldots$ & $5190(80)$ & 0.48 & $\ldots$ & $\ldots$ \\
\hline 12 & $\ldots$ & $\cdots$ & $\ldots$ & $\ldots$ & $\cdots$ & $\cdots$ & $\ldots$ & $\cdots$ & $4240(210)$ & 0.04 \\
\hline 13 & $\cdots$ & $\cdots$ & $\cdots$ & $\cdots$ & $\cdots$ & $\cdots$ & $\cdots$ & $\cdots$ & $\ldots$ & $\cdots$ \\
\hline 14 & $\cdots$ & $\cdots$ & $\cdots$ & $\cdots$ & $\cdots$ & $\cdots$ & $\cdots$ & $\cdots$ & $12230(220)$ & 0.09 \\
\hline 15 & $\ldots$ & $\cdots$ & $\ldots$ & $\cdots$ & $\ldots$ & $\cdots$ & $\ldots$ & $\cdots$ & & \\
\hline 16 & $7870(50)$ & 0.36 & $6630(120)$ & 0.55 & $6160(80)$ & 0.56 & $5870(90)$ & 0.60 & $5240(80)$ & 0.54 \\
\hline 17 & $\ldots$ & $\cdots$ & $5470(250)$ & 0.09 & $4850(200)$ & 0.13 & $4670(130)$ & 0.15 & $4580(60)$ & 0.19 \\
\hline 18 & $7130(90)$ & 0.11 & $5690(80)$ & 0.26 & $5120(70)^{\prime}$ & 0.31 & $4900(80)^{\prime}$ & 0.34 & $4630(50)$ & 0.36 \\
\hline 19 & $7250(60)$ & 0.22 & $5540(90)$ & 0.37 & $5200(130)$ & 0.41 & $5050(100)$ & 0.44 & $4660(60)$ & 0.48 \\
\hline 20 & $\ldots$ & $\ldots$ & $\ldots$ & $\ldots$ & $\ldots$ & $\ldots$ & $\ldots$ & $\ldots$ & 4360 (110) & 0.04 \\
\hline 21 & $\ldots$ & $\ldots$ & $\ldots$ & $\ldots$ & $\ldots$ & $\ldots$ & $\ldots$ & $\ldots$ & $4370(210)$ & 0.05 \\
\hline 22 & $7240(360)$ & 0.01 & $5320(120)$ & 0.09 & $4890(290)$ & 0.11 & $4870(220)$ & 0.14 & $4410(150)$ & 0.11 \\
\hline 23 & $\ldots$ & $\cdots$ & $\ldots$ & $\cdots$ & $\ldots$ & $\cdots$ & $\ldots$ & $\cdots$ & $\ldots$ & $\cdots$ \\
\hline 24 & $\cdots$ & $\cdots$ & $\ldots$ & $\cdots$ & $4930(230)$ & 0.04 & $\ldots$ & $\cdots$ & $\ldots$ & $\cdots$ \\
\hline 25 & $\cdots$ & $\cdots$ & $5510(350)$ & 0.07 & $4840(330)$ & 0.07 & $4650(90)$ & 0.10 & $4450(70)$ & 0.11 \\
\hline 26 & $\ldots$ & $\ldots$ & $\ldots$ & $\ldots$ & $\ldots$ & $\ldots$ & $\ldots$ & $\ldots$ & $\ldots$ & $\ldots$ \\
\hline 27 & $\cdots$ & $\cdots$ & $\cdots$ & $\cdots$ & $\cdots$ & $\cdots$ & $\cdots$ & $\cdots$ & $\cdots$ & $\cdots$ \\
\hline 28 & $\cdots$ & $\cdots$ & $\ldots$ & $\cdots$ & $\ldots$ & $\cdots$ & $\ldots$ & $\cdots$ & $\ldots$ & $\cdots$ \\
\hline 29 & $\cdots$ & $\cdots$ & $5620(220)$ & 0.10 & $5170(170)$ & 0.16 & $4790(80)$ & 0.21 & $4470(50)$ & 0.29 \\
\hline 30 & $\ldots$ & $\ldots$ & $\ldots$ & $\ldots$ & $\ldots$ & $\ldots$ & $\ldots$ & $\ldots$ & $4270(190)$ & 0.04 \\
\hline 31 & $\ldots$ & $\ldots$ & $\cdots$ & $\ldots$ & $5450(180)$ & 0.06 & $4900(110)$ & 0.06 & $4630(110)$ & 0.07 \\
\hline 32 & $6620(110)$ & 0.07 & $\cdots$ & $\cdots$ & $\ldots$ & $\ldots$ & $\ldots$ & $\ldots$ & $\ldots$ & $\ldots$ \\
\hline 33 & $\cdots$ & $\cdots$ & $\cdots$ & $\cdots$ & $\cdots$ & $\cdots$ & $\cdots$ & $\cdots$ & $\ldots$ & $\cdots$ \\
\hline 34 & $8880(40)$ & 0.48 & $7680(130)$ & 0.70 & $7160(30)$ & 0.70 & $7100(50)$ & 0.75 & $6650(40)$ & 0.76 \\
\hline 35 & $\cdots$ & $\cdots$ & $\cdots$ & $\cdots$ & $\ldots$ & $\cdots$ & $\ldots$ & $\cdots$ & $\ldots$ & $\cdots$ \\
\hline 36 & $\cdots$ & $\cdots$ & $\cdots$ & $\cdots$ & $\cdots$ & $\cdots$ & $\cdots$ & $\cdots$ & $\cdots$ & $\cdots$ \\
\hline
\end{tabular}


Table 5 - Continued.

\begin{tabular}{|c|c|c|c|c|c|c|c|c|c|c|c|}
\hline \multirow[b]{2}{*}{ Line } & \multicolumn{2}{|c|}{ Day 34} & \multicolumn{2}{|c|}{ Day 37} & \multicolumn{2}{|c|}{ Day 40} & \multicolumn{2}{|c|}{ Day 44} & \multicolumn{3}{|c|}{ Day 47} \\
\hline & Vel. & Depth & Vel. & Depth & Vel. & Depth & Vel. & Depth & Vel. & Depth & \\
\hline 1 & $\cdots$ & $\cdots$ & $\cdots$ & $\cdots$ & $\cdots$ & $\cdots$ & $\cdots$ & $\cdots$ & $\cdots$ & $\cdots$ & \\
\hline 2 & $\ldots$ & $\ldots$ & $\ldots$ & $\ldots$ & $\ldots$ & $\ldots$ & $\ldots$ & $\ldots$ & $\ldots$ & $\ldots$ & \\
\hline 3 & $3210(370)$ & $\ldots$ & $\cdots$ & $\ldots$ & $\cdots$ & $\cdots$ & $3510(100)$ & 0.35 & $3350(80)$ & 0.37 & \\
\hline 4 & 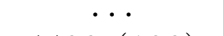 & $\cdots$ & $\cdots$ & $\cdots$ & $\cdots$ & $\cdots$ & $\cdots$ & & & & \\
\hline 5 & $4400(130)$ & 0.22 & $4220(100)$ & 0.17 & $\cdots$ & $\cdots$ & $4180(190)$ & 0.28 & $3870(80)$ & 0.24 & \\
\hline 6 & $4340(100)$ & 0.24 & $4410(100)$ & 0.20 & $\ldots$ & $\cdots$ & $3990(110)$ & 0.29 & $4000(90)$ & 0.35 & \\
\hline 7 & $3600(230)$ & 0.44 & $3550(140)$ & 0.39 & $3660(60)$ & 0.35 & $3370(80)^{\prime}$ & 0.42 & $3100(220)$ & 0.40 & \\
\hline 8 & $\ldots$ & $\cdots$ & $\ldots$ & & $\ldots$ & $\cdots$ & & & & & \\
\hline 9 & $3810(170)$ & 0.19 & $3690(80)$ & 0.14 & $3730(60)$ & 0.23 & $3650(100)$ & 0.27 & $3650(130)$ & 0.24 & \\
\hline 10 & {$[4394.6(3.4)]$} & 0.08 & {$[4395.5(3.4)]$} & 0.06 & {$[4405.2(0.9)]$} & 0.09 & {$[4404.1(1.9)]$} & 0.09 & $\ldots$ & $\ldots$ & \\
\hline 11 & $4220(50)$ & 0.51 & $3810(90)$ & 0.54 & $3580(70)$ & 0.59 & $3630(70)$ & 0.56 & $3700(120)$ & 0.56 & \\
\hline 12 & $4020(110)$ & 0.07 & 3540 (190) & 0.08 & $3480(60)$ & 0.12 & $3540(160)$ & 0.12 & $3410(80)^{\prime}$ & 0.12 & \\
\hline 13 & $3900(100)$ & 0.02 & $3740(280)$ & 0.04 & $3660(60)$ & 0.07 & $3660(80)$ & 0.08 & $3410(70)$ & 0.10 & \\
\hline 14 & $11960(90)$ & 0.08 & $11710(80)$ & 0.08 & $11700(60)$ & 0.11 & $\ldots$ & $\ldots$ & $11650(100)$ & 0.12 & I \\
\hline 15 & $\ldots$ & $\ldots$ & $\ldots$ & $\ldots$ & {$[4748.0(0.9)]$} & 0.05 & $\ldots$ & $\ldots$ & {$[4753.2(1.6)]$} & 0.07 & $\stackrel{\oplus}{\mapsto}$ \\
\hline 16 & $5050(40)$ & 0.50 & $4270(120)$ & 0.48 & $4160(60)$ & 0.49 & $4020(50)$ & 0.42 & $3870(50)$ & 0.32 & । \\
\hline 17 & $4270(50)$ & 0.25 & $3750(110)$ & 0.25 & $3790(50)$ & 0.33 & $3840(50)$ & 0.33 & $3720(50)$ & 0.35 & \\
\hline 18 & $4310(80)$ & 0.42 & $3800(80)$ & 0.46 & $3720(50)$ & 0.51 & $3770(90)$ & 0.54 & $3680(50)$ & 0.54 & \\
\hline 19 & $4410(80)$ & 0.49 & 3910 (130) & 0.55 & $3890(70)$ & 0.56 & 3960 (120) & 0.55 & $3870(50)$ & 0.54 & \\
\hline 20 & $4170(150)$ & 0.03 & $3720(110)$ & 0.04 & $3680(60)$ & 0.06 & $3770(90)^{\prime}$ & 0.08 & $3420(70)$ & 0.08 & \\
\hline 21 & $4040(150)$ & 0.04 & $3590(150)$ & 0.05 & $3610(70)$ & 0.09 & $3430(60)$ & 0.09 & $3510(90)$ & 0.11 & \\
\hline 22 & $4090(50)$ & 0.12 & $3560(140)$ & 0.14 & $3620(60)$ & 0.16 & $3680(110)$ & 0.16 & $3480(90)$ & 0.17 & \\
\hline 23 & $\ldots$ & $\ldots$ & $\ldots$ & $\ldots$ & $3440(60)$ & 0.02 & $\ldots$ & $\ldots$ & $\ldots$ & $\ldots$ & \\
\hline 24 & $3980(190)$ & 0.03 & $3340(110)$ & 0.05 & $3430(90)$ & 0.07 & $3450(50)$ & 0.10 & $3240(80)$ & 0.09 & \\
\hline 25 & $4140(50)^{\prime}$ & 0.16 & $3660(140)$ & 0.19 & $3650(60)$ & 0.23 & $3610(50)$ & 0.27 & $3440(50)$ & 0.27 & \\
\hline 26 & $4290(60)$ & 0.15 & $3690(130)$ & 0.18 & $3710(80)$ & 0.24 & $3610(40)$ & 0.27 & $3410(40)$ & 0.30 & \\
\hline 27 & $12010(110)$ & 0.03 & $11670(310)$ & 0.02 & $11820(60)$ & 0.02 & $\ldots$ & $\cdots$ & $\ldots$ & $\cdots$ & \\
\hline 28 & $\cdots$ & $\cdots$ & & $\cdots$ & $\cdots$ & $\cdots$ & $\begin{array}{c}\cdots \\
250\end{array}$ & $\cdots$ & $\cdots$ & $\cdots$ & \\
\hline 29 & $4170(50)$ & 0.38 & $3750(80)$ & 0.43 & $3680(70)$ & 0.49 & $3580(40)$ & 0.51 & $3540(40)$ & 0.52 & \\
\hline 30 & $3770(90)$ & 0.09 & $3620(190)$ & 0.08 & $3510(70)$ & 0.11 & $3350(60)$ & 0.14 & $3150(40)$ & 0.17 & \\
\hline 31 & $4200(60)$ & 0.12 & $4010(120)$ & 0.13 & $3740(80)$ & 0.17 & $3750(50)$ & 0.19 & $3640(60)$ & 0.22 & \\
\hline 32 & $4050(70)$ & 0.05 & $\ldots$ & $\cdots$ & $3760(50)$ & 0.03 & $\ldots$ & $\cdots$ & $\ldots$ & $\cdots$ & \\
\hline 33 & $11900(50)$ & 0.10 & $11760(80)$ & 0.07 & $11770(40)$ & 0.13 & $11730(50)$ & 0.11 & $11730(50)$ & 0.14 & \\
\hline 34 & $6230(40)^{\prime}$ & 0.77 & $5860(190)$ & 0.78 & $5570(60)$ & 0.78 & $5120(50)$ & 0.76 & $4940(40)$ & 0.74 & \\
\hline 35 & $\ldots$ & $\ldots$ & $4870(180)$ & 0.78 & $\ldots$ & $\ldots$ & $4780(60)$ & 0.59 & $4470(120)$ & 0.57 & \\
\hline 36 & $\cdots$ & $\cdots$ & $4880(150)$ & 0.44 & $\cdots$ & $\cdots$ & $4930(330)$ & 0.24 & $4340(160)$ & 0.35 & \\
\hline
\end{tabular}


Table 5 - Continued.

\begin{tabular}{|c|c|c|c|c|c|c|c|c|c|c|c|}
\hline \multirow[b]{2}{*}{ Line } & \multicolumn{2}{|c|}{ Day 49} & \multicolumn{2}{|c|}{ Day 76} & \multicolumn{2}{|c|}{ Day 95} & \multicolumn{2}{|c|}{ Day 96} & \multicolumn{3}{|c|}{ Day 124} \\
\hline & Vel. & Depth & Vel. & Depth & Vel. & Depth & Vel. & Depth & Vel. & Depth & \\
\hline 1 & $\cdots$ & $\cdots$ & $\cdots$ & $\cdots$ & $\cdots$ & $\cdots$ & $\cdots$ & $\cdots$ & $\cdots$ & $\cdots$ & \\
\hline 2 & $\ldots$ & $\ldots$ & $\ldots$ & $\ldots$ & $\ldots$ & $\ldots$ & $\ldots$ & $\ldots$ & $\ldots$ & $\ldots$ & \\
\hline 3 & $\ldots$ & $\ldots$ & $3660(290)$ & 0.70 & $\ldots$ & $\ldots$ & $\ldots$ & $\ldots$ & $3270(230)$ & 0.51 & \\
\hline 4 & $\cdots$ & $\cdots$ & $\ldots$ & $\cdots$ & $\cdots$ & $\cdots$ & $\cdots$ & $\cdots$ & $\ldots$ & $\cdots$ & \\
\hline 5 & $\ldots$ & $\ldots$ & $\ldots$ & $\ldots$ & $\cdots$ & $\ldots$ & $\cdots$ & $\ldots$ & $\ldots$ & $\cdots$ & \\
\hline 6 & $\ldots$ & $\ldots$ & $\ldots$ & $\ldots$ & $\ldots$ & $\ldots$ & $\ldots$ & $\ldots$ & $\ldots$ & $\ldots$ & \\
\hline 7 & $3230(90)$ & 0.28 & $2980(290)$ & 0.47 & $\cdots$ & $\ldots$ & $2320(420)$ & 0.42 & $2250(240)$ & 0.23 & \\
\hline 8 & & & & & $\ldots$ & $\ldots$ & $\ldots$ & $\ldots$ & & & \\
\hline 9 & $3610(80)$ & 0.26 & $3290(330)$ & 0.27 & $\ldots$ & $\ldots$ & $\ldots$ & $\ldots$ & $2820(250)$ & 0.33 & \\
\hline 10 & {$[4407.7(1.1)]$} & 0.12 & $\ldots$ & $\ldots$ & $\ldots$ & $\ldots$ & $\ldots$ & $\ldots$ & {$[4422.6(1.7)]$} & 0.22 & \\
\hline 11 & $3410(70)$ & 0.62 & $2790(160)$ & 0.69 & $\ldots$ & $\ldots$ & $2450(500)$ & 0.66 & $2350(80)$ & 0.66 & \\
\hline 12 & $3220(40)$ & 0.17 & $2290(70)^{\prime}$ & 0.22 & $2010(90)$ & 0.34 & $1900(100)$ & 0.38 & $\ldots$ & $\cdots$ & \\
\hline 13 & $3280(40)$ & 0.14 & $2310(60)$ & 0.21 & $1850(90)$ & 0.31 & $2060(80)^{\prime}$ & 0.23 & $\ldots$ & $\ldots$ & \\
\hline 14 & $11370(40)$ & 0.13 & $10550(170)$ & 0.12 & $11240(100)$ & 0.11 & $10110(440)$ & 0.10 & $10700(90)$ & 0.13 & \\
\hline 15 & {$[4753.5(0.7)]$} & 0.07 & {$[4765.0(1.6)]$} & 0.25 & {$[4755.5(1.6)]$} & 0.05 & {$[4775.2(1.9)]$} & 0.19 & {$[4783.2(0.9)]$} & 0.12 & 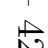 \\
\hline 16 & $3520(40)$ & 0.32 & 2310 & 0.28 & $2060(50)$ & 0.39 & 2120 & 0.34 & $2010(40)$ & 0.42 & $\stackrel{N}{N}$ \\
\hline 17 & $3410(40)$ & 0.40 & $2550(60)$ & 0.50 & $2240(70)$ & 0.61 & $2330(80)^{\prime}$ & 0.61 & $1830(50)$ & 0.52 & \\
\hline 18 & $3440(40)$ & 0.59 & $2790(50)$ & 0.70 & $2500(60)$ & 0.76 & $2600(70)$ & 0.73 & $2030(40)$ & 0.58 & \\
\hline 19 & $3660(40)$ & 0.59 & $2900(130)$ & 0.70 & $2660(110)$ & 0.64 & $2660(190)$ & 0.64 & $1960(40)$ & 0.62 & \\
\hline 20 & $3330(40)$ & 0.09 & $\ldots$ & $\ldots$ & $2100(110)$ & 0.16 & $2110(200)$ & 0.17 & $\ldots$ & $\ldots$ & \\
\hline 21 & $3230(40)$ & 0.13 & $\ldots$ & $\ldots$ & $2110(80)$ & 0.25 & $2120(160)$ & 0.21 & $\ldots$ & $\ldots$ & \\
\hline 22 & $3240(40)$ & 0.19 & $2620(290)$ & 0.42 & $1950(40)$ & 0.35 & $2030(200)$ & 0.32 & $1710(30)$ & 0.36 & \\
\hline 23 & $3110(40)$ & 0.04 & $\ldots$ & $\ldots$ & $1570(50)$ & 0.14 & $\ldots$ & $\ldots$ & $\ldots$ & $\ldots$ & \\
\hline 24 & $3190(50)$ & 0.11 & $2240(110)$ & 0.20 & $1850(40)$ & 0.24 & $1970(80)$ & 0.22 & $1340(40)$ & 0.42 & \\
\hline 25 & $3320(30)$ & 0.32 & $2460(70)$ & 0.44 & $2140(50)$ & 0.51 & $2180(60)$ & 0.47 & $1450(40)$ & 0.17 & \\
\hline 26 & $3360(40)$ & 0.35 & $2580(60)$ & 0.52 & $2360(30)$ & 0.61 & $2250(60)$ & 0.58 & $1790(40)$ & 0.40 & \\
\hline 27 & $11780(50)$ & 0.02 & ... & $\ldots$ & ... & $\ldots$ & $\ldots$ & $\ldots$ & ... & $\ldots$ & \\
\hline 28 & & & $\ldots$ & $\cdots$ & $\cdots$ & $\ldots$ & $\ldots$ & $\cdots$ & $\cdots$ & $\cdots$ & \\
\hline 29 & $3370(30)$ & 0.59 & $3010(90)$ & 0.70 & $3240(30)$ & 0.76 & $3140(60)$ & 0.76 & $5620(40)$ & 0.89 & \\
\hline 30 & $3100(30)$ & 0.19 & $2610(70)$ & 0.41 & $2640(40)$ & 0.52 & $2410(80)$ & 0.53 & $2720(30)$ & 0.60 & \\
\hline 31 & $3470(30)$ & 0.25 & $2800(50)$ & 0.38 & $2350(40)$ & 0.46 & $2190(50)$ & 0.45 & $1770(40)$ & 0.31 & \\
\hline 32 & & & & & & $\ldots$ & & $\ldots$ & & & \\
\hline 33 & $11640(30)$ & 0.15 & $11300(90)$ & 0.17 & $\ldots$ & $\ldots$ & $\ldots$ & $\ldots$ & $\ldots$ & $\ldots$ & \\
\hline 34 & $4830(80)$ & 0.73 & $5440(120)$ & 0.63 & $5100(610)$ & 0.65 & $5600(150)$ & 0.64 & $4440(50)$ & 0.83 & \\
\hline 35 & $\ldots$ & $\ldots$ & $4150(260)$ & 0.85 & $\ldots$ & $\ldots$ & $\ldots$ & $\ldots$ & $2890(130)$ & 0.82 & \\
\hline 36 & $\cdots$ & $\cdots$ & $3660(160)$ & 0.54 & $\cdots$ & $\cdots$ & $\cdots$ & $\cdots$ & $\ldots$ & $\ldots$ & \\
\hline
\end{tabular}


Table 5 - Continued.

\begin{tabular}{|c|c|c|c|c|c|c|c|c|c|c|}
\hline \multirow[b]{2}{*}{ Line } & \multicolumn{2}{|c|}{ Day 138} & \multicolumn{2}{|c|}{ Day 159} & \multicolumn{2}{|c|}{ Day 163} & \multicolumn{2}{|c|}{ Day 313} & \multicolumn{2}{|c|}{ Day 333} \\
\hline & Vel. & Depth & Vel. & Depth & Vel. & Depth & Vel. & Depth & Vel. & Depth \\
\hline 1 & $\cdots$ & $\cdots$ & $\cdots$ & $\cdots$ & $\cdots$ & $\cdots$ & $\cdots$ & $\cdots$ & $\cdots$ & $\cdots$ \\
\hline 2 & $\ldots$ & $\ldots$ & $\ldots$ & $\ldots$ & $\ldots$ & $\ldots$ & $\ldots$ & $\ldots$ & $\ldots$ & $\ldots$ \\
\hline 3 & $\cdots$ & $\cdots$ & $\cdots$ & $\cdots$ & $\cdots$ & $\cdots$ & $\cdots$ & $\cdots$ & $\cdots$ & $\cdots$ \\
\hline 4 & $\cdots$ & $\cdots$ & $\cdots$ & $\cdots$ & $\cdots$ & $\cdots$ & $\cdots$ & $\cdots$ & $\cdots$ & $\cdots$ \\
\hline 5 & $\cdots$ & $\cdots$ & $\ldots$ & $\ldots$ & $\ldots$ & $\ldots$ & $\ldots$ & $\ldots$ & $\ldots$ & $\ldots$ \\
\hline 6 & $\cdots$ & $\cdots$ & $\cdots$ & $\cdots$ & $\cdots$ & $\cdots$ & $\cdots$ & $\cdots$ & $\cdots$ & $\cdots$ \\
\hline 7 & $\ldots$ & $\ldots$ & $\ldots$ & $\ldots$ & $\ldots$ & $\ldots$ & $\ldots$ & $\ldots$ & $\ldots$ & $\ldots$ \\
\hline 8 & $\cdots$ & $\cdots$ & $\cdots$ & $\cdots$ & $\cdots$ & $\cdots$ & $\cdots$ & $\cdots$ & $\cdots$ & $\cdots$ \\
\hline 9 & $\cdots$ & $\ldots$ & $\ldots$ & $\ldots$ & $\ldots$ & $\cdots$ & $\cdots$ & $\ldots$ & $1800(190)$ & 0.39 \\
\hline 10 & $\ldots$ & $\ldots$ & $\ldots$ & $\ldots$ & $\ldots$ & $\ldots$ & $\ldots$ & $\ldots$ & {$[4436.9(3.7)]$} & 0.28 \\
\hline 11 & $1800(140)$ & 0.62 & $1950(190)$ & 0.61 & $1620(140)$ & 0.57 & $1290(390)$ & 0.67 & $2280(190)$ & 0.50 \\
\hline 12 & $\cdots$ & $\cdots$ & $\cdots$ & $\cdots$ & $\cdots$ & $\cdots$ & $\ldots$ & $\cdots$ & $\cdots$ & $\cdots$ \\
\hline 13 & $\cdots$ & $\cdots$ & $\ldots$ & $\cdots$ & $\ldots$ & $\cdots$ & $\cdots$ & $\cdots$ & $\cdots$ & $\cdots$ \\
\hline 14 & $\cdots$ & $\ldots$ & $10760(70)$ & 0.12 & $10780(110)$ & 0.11 & $\cdots$ & $\cdots$ & $\cdots$ & $\cdots$ \\
\hline 15 & $\ldots$ & $\cdots$ & $\ldots$ & $\cdots$ & $\ldots$ & $\cdots$ & $\ldots$ & $\cdots$ & $\ldots$ & $\cdots$ \\
\hline 16 & $2190(60)$ & 0.75 & $2360(70)$ & 0.85 & $2490(60)$ & 0.84 & $2190(240)$ & 1.05 & $2370(80)$ & 0.86 \\
\hline 17 & $1790(100)$ & 0.53 & $1510(40)$ & 0.53 & $1530(50)$ & 0.48 & $1500(100)$ & 0.58 & $1520(60)$ & 0.36 \\
\hline 18 & $1910(130)$ & 0.54 & $1780(40)$ & 0.59 & $1780(50)$ & 0.58 & $1780(250)$ & 0.66 & $1910(80)$ & 0.60 \\
\hline 19 & $1430(220)$ & 0.67 & $960(40)^{\prime}$ & 0.67 & $1000(120)$ & 0.65 & $1910(180)$ & 0.30 & $1910(90)$ & 0.24 \\
\hline 20 & $\cdots$ & $\cdots$ & $\ldots$ & $\cdots$ & $\ldots$ & $\cdots$ & $\ldots$ & $\ldots$ & ... & $\cdots$ \\
\hline 21 & $\cdots$ & $\cdots$ & $\cdots$ & $\cdots$ & $\cdots$ & $\cdots$ & $\cdots$ & $\cdots$ & $\cdots$ & $\cdots$ \\
\hline 22 & $1500(130)$ & 0.41 & $\cdots$ & $\cdots$ & $1430(80)$ & 0.38 & $\cdots$ & $\cdots$ & $\cdots$ & $\cdots$ \\
\hline 23 & & $\cdots$ & $\cdots$ & $\cdots$ & & $\cdots$ & $\cdots$ & $\cdots$ & $\cdots$ & $\cdots$ \\
\hline 24 & $1510(300)$ & 0.40 & $\ldots$ & $\ldots$ & $1150(70)$ & 0.41 & $\cdots$ & $\cdots$ & $\ldots$ & $\ldots$ \\
\hline 25 & $1260(80)^{\prime}$ & 0.18 & $\cdots$ & $\cdots$ & $1290(60)$ & 0.17 & $\ldots$ & $\cdots$ & $\ldots$ & $\ldots$ \\
\hline 26 & $1630(70)$ & 0.44 & $\ldots$ & $\ldots$ & $1500(90)$ & 0.39 & $2450(170)$ & 0.42 & $2260(90)$ & 0.48 \\
\hline 27 & $\ldots$ & $\ldots$ & $10500(100)$ & 0.06 & $\ldots$ & $\ldots$ & $\ldots$ & $\cdots$ & $\ldots$ & $\cdots$ \\
\hline 28 & $\ldots$ & $\cdots$ & $\ldots$ & $\cdots$ & $\ldots$ & $\cdots$ & $\ldots$ & $\cdots$ & $\ldots$ & $\cdots$ \\
\hline 29 & $5330(50)$ & 0.91 & $5650(160)$ & 0.92 & $5480(130)$ & 0.91 & $4840(430)$ & 0.95 & $4060(460)$ & 0.91 \\
\hline 30 & $2660(60)$ & 0.58 & $2730(60)$ & 0.49 & $2770(80)$ & 0.46 & $4540(270)$ & 0.54 & $4930(150)$ & 0.53 \\
\hline 31 & $1660(170)$ & 0.30 & $1610(30)$ & 0.39 & $1630(50)$ & 0.38 & $1770(290)$ & 0.61 & $1700(80)^{\prime}$ & 0.60 \\
\hline $\begin{array}{l}32 \\
2 ?\end{array}$ & $\cdots$ & $\cdots$ & $\cdots$ & $\cdots$ & $\cdots$ & $\cdots$ & $\cdots$ & $\cdots$ & $\cdots$ & $\cdots$ \\
\hline 33 & $\cdots$ & $\cdots$ & $\cdots$ & $\cdots$ & $\cdots$ & $\cdots$ & $\cdots$ & $\cdots$ & $\cdots$ & $\cdots$ \\
\hline 34 & $3740(110)$ & 0.88 & $3580(30)$ & 0.92 & $3630(90)$ & 0.92 & $3460(70)$ & 0.98 & $3520(30)$ & 0.96 \\
\hline
\end{tabular}


Table 5 - Continued.

\begin{tabular}{rcccccccccc}
\hline \hline \multirow{2}{*}{ Line } & \multicolumn{2}{c}{ Day 138 } & \multicolumn{2}{c}{ Day 159 } & \multicolumn{2}{c}{ Day 163 } & \multicolumn{2}{c}{ Day 313 } & \multicolumn{3}{c}{ Day 333 } \\
\cline { 2 - 11 } & Vel. & Depth & Vel. & Depth & Vel. & Depth & Vel. & Depth & Vel. & Depth \\
\hline 35 & $2980(40)$ & 0.93 & $\ldots$ & $\ldots$ & $2910(180)$ & 0.88 & $2440(190)$ & 0.91 & $\ldots$ & $\ldots$ \\
36 & $2970(40)$ & 0.63 & $\ldots$ & $\ldots$ & $2660(50)$ & 0.65 & $2250(110)$ & 0.84 & $\ldots$ & $\ldots$ \\
\hline
\end{tabular}

Note. - Line velocities for absorption features measured directly from spectra of SN 1999em with estimated $1 \sigma$ statistical uncertainty shown in parentheses, shown for four example days only. Uncertainty includes measurement error and uncertainty in the wavelength scale, but does not include uncertainty in the rest wavelength estimation, which may be significant for blended features. All spectra were deredshifted by $800 \mathrm{~km} \mathrm{~s}^{-1}$ prior to the velocity determination. Note that the emission-dominated nebular spectrum from day 517 is not included in the tabulation.

${ }^{a}$ Day since discovery, 1999 October 29 (HJD 2,451,480.94), rounded to the nearest day.

${ }^{\mathrm{b}}$ Line number corresponding to feature indicated in Figures 9 and 10, and identified in Table 4.

${ }^{\mathrm{c}}$ Main ion responsible for feature. Colon indicates significant blending of multiple ions; see Table 4 for components of blend. Velocities derived using blended lines may not yield accurate photospheric velocities, since the relative strengths of the components may change with time.

${ }^{\mathrm{d}}$ Rest wavelength (air) adopted for line feature. In the case of blended lines, a weighted average of the blended components is used; see text for details.

eVelocity measured in units of $\mathrm{km} \mathrm{s}^{-1}$, with uncertainty listed in parentheses. For line features lacking identification, the measured rest wavelength and uncertainty are given in brackets.

${ }^{\mathrm{f}}$ Depth is defined as: $d=1-f_{\min }$, where $f_{\min }$ is the normalized flux level at the location of the line's minimum with the continuum set to 1 . It should be noted that the measured depth in some cases may depend on the strength of adjacent features since this affects the location of the points used to fit the normalizing continuum.

${ }^{g}$ To maintain consistency with the method traditionally used to determine the photospheric velocity by averaging the velocities derived from Fe II $\lambda \lambda 4924,5018,5169$ line measurements, we adopt the rest wavelength of Fe II $\lambda 4924$ to determine this feature's velocity. The consistently lower velocity derived compared with the other Fe II lines of the multiplet, however, suggests significant blending with Ba II $\lambda 4934$.

${ }^{\mathrm{h}}$ Becomes weak and probably blended with $\mathrm{Na}$ I D by day 10. 


\subsection{The Reddening of SN $1999 \mathrm{em}$}

SN $1999 \mathrm{em}$ suffers from minimal Galactic reddening, $E(B-V)_{\mathrm{MW}}=0.04$ mag (Schlegel, Finkbeiner, \& Davis 1998). From theoretical fits to the early-time continuum shape, Baron et al. (2000) estimate the total (Galactic + host galaxy) reddening of SN 1999em to be $0.05 \lesssim$ $E(B-V)_{\text {tot }} \lesssim 0.10 \mathrm{mag}$, with an upper limit of $E(B-V)_{\text {tot }}<0.15$ mag; the most successful fits seem to favor $E(B-V)_{\text {tot }}=0.10$ mag. We checked the consistency of this result using two techniques.

First, we compared the BVI color of SN $1999 \mathrm{em}$ with that of SN $1987 \mathrm{~A}$ at the moment the He I $\lambda 5876$ line disappears from both objects' spectra. Since exciting a significant amount of He I to the high energy level $(E=20.97 \mathrm{eV}$; see Table 4$)$ needed to produce the $\lambda 5876$ line is very temperature sensitive (generally greater than about $T_{\text {eff }}=10,000 \mathrm{~K}$; Hatano et al. 1999; Branch 1987; see also Figure 1), we expect this line to disappear when the photosphere is at a well-defined temperature. Therefore, the intrinsic color of the two objects should be similar at this early phase, before significant line blanketing from metal lines begins. We assume the reddening of SN 1987A to be $E(B-V)=0.15 \pm 0.05$ mag (see discussion in Arnett et al. 1989) and compare its color at this phase with that of SN $1999 \mathrm{em}$. For SN $1999 \mathrm{em}$, the He I $\lambda 5876$ line becomes too weak to measure $10 \pm 1$ days after discovery, roughly corresponding with the epoch when the Fe II $\lambda 5169$ absorption first appears (strengthening the argument that at this phase the optical spectrum is nearly a blackbody, with little line blanketing). At that epoch, its BVI magnitudes are 14.01, 13.85, and 13.49, respectively, interpolated from the values given in Table 2. Phillips et al. (1988) estimate that the He I $\lambda 5876$ line disappeared in the spectrum of SN 1987A on JD 2,446,854, just four days after discovery. From the photometry of Hamuy et al. (1988), the BVI magnitudes for SN 1987A on that date are 4.83,4.45, and 4.01, respectively. Using the reddening law of Savage \& Mathis (1979) and minimizing the color difference between the two SNe at this epoch, we derive a reddening of $E(B-V)=0.03 \pm 0.05 \mathrm{mag}$ for $\mathrm{SN} 1999 \mathrm{em}$, in good agreement with the estimate of Baron et al. (2000).

A second method sometimes used to provide a reddening estimate for SNe II-P (e.g., Schmidt et al. 1993) is to assume that the early-time color evolution of all SNe II-P is similar, and compare the early color evolution of a SN II-P with that observed in SN 1969L, a Type II-P believed to suffer nearly zero host-galaxy reddening (Kirshner \& Kwan 1974; Schmidt et al. 1992), and only $E(B-V)_{\mathrm{MW}}=0.06 \mathrm{mag}$ (Schlegel et al. 1998). Using the time of explosion for SN 1999em derived in $\S 3(5.3 \pm 1.4$ days before discovery), Figure 13 shows that at early times, SN $1999 \mathrm{em}$ and SN 1969L have a very similar color. This suggests similarly low reddenings for the two events. We do note, however, that the $B-V$ color evolution of SN $1999 \mathrm{em}$ at later times deviates substantially from that of SN 1969L, and may indicate that SN 1999em's subsequent color evolution differed somewhat from the norm. Although limited by the small number of observations and rather poor temporal coverage, Schmidt et al. (1992) demonstrate that the $B-V$ color evolution of the 4 SNe II-P in their sample is quite similar to that of SN 1969L during the recombination phase

(none has photometric data taken prior to 14 days after the explosion, however). It is possible 


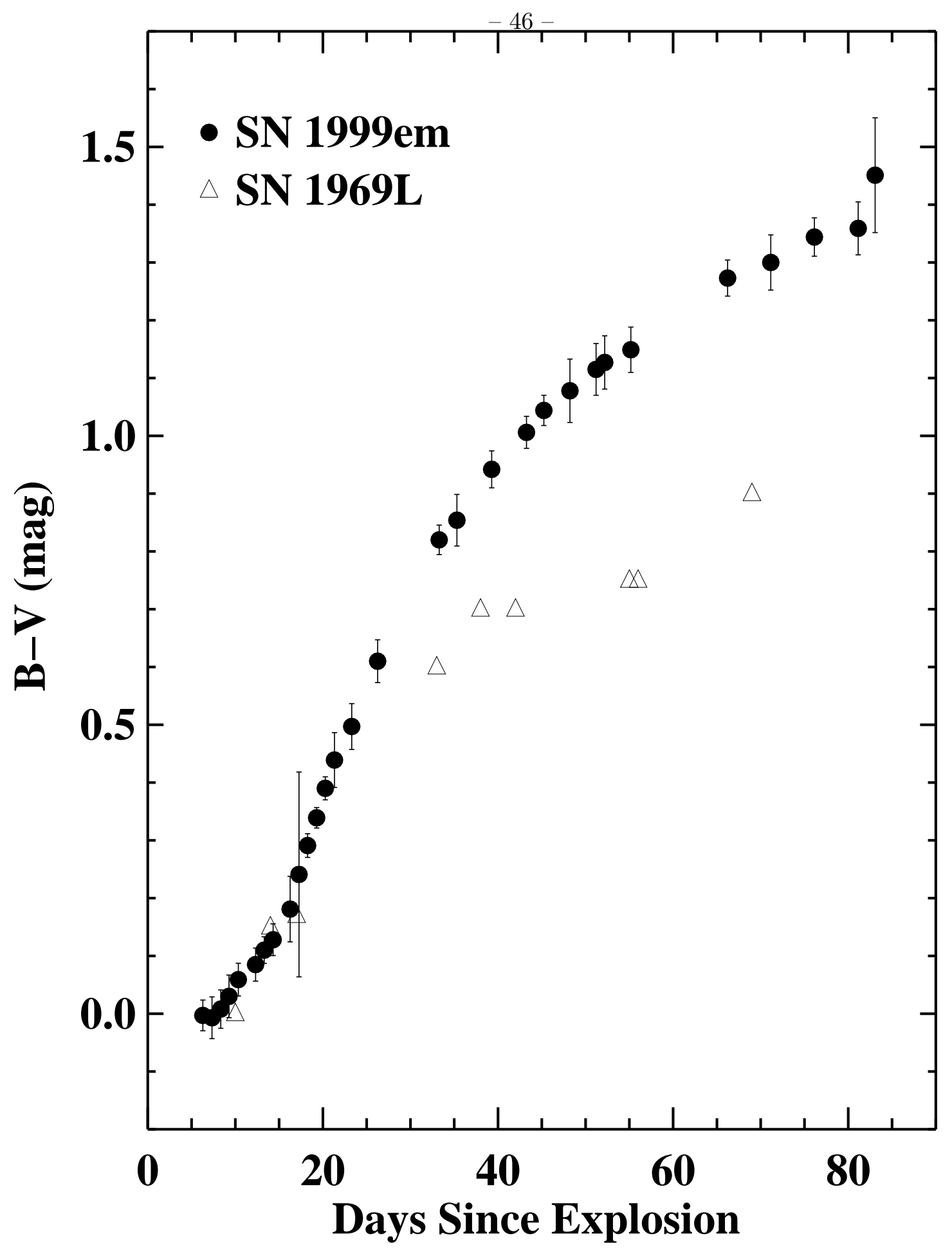

Fig. 13. - The temporal evolution of the $B-V$ color of SN 1999em compared with that of the prototypical Type II-P event, SN 1969L, which is believed to be nearly unreddened. The time of explosion for both events is derived through the EPM analysis (see $\S 3$ for SN 1999em and Schmidt et al. [1992] for SN 1969L). The good agreement between the early-time color evolution of the two events suggests that SN 1999em might also suffer little reddening. 


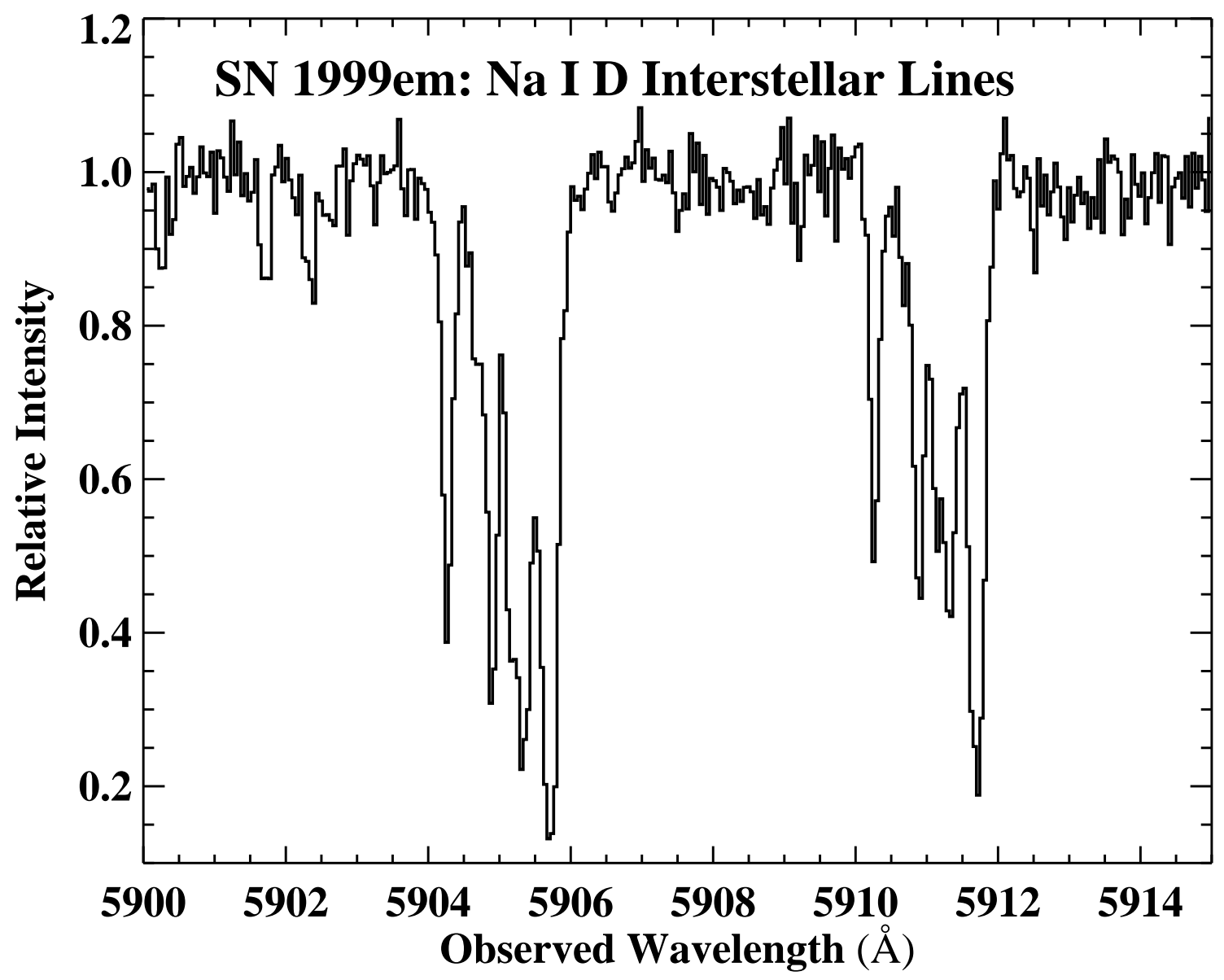

Fig. 14. - Interstellar Na I D $(\lambda \lambda 5890,5896)$ absorption by gas in NGC 1637 along the line of sight to SN 1999em. 
that the large deviation between the later color evolution of SN 1999em and SN 1969L indicates a real difference between SN 1999em and this prototypical SN II-P. We shall further investigate the possibility of unusual photometric behavior for SN 1999em in $\S 4.4$.

A final indication that there may be little reddening for SN 1999em comes from the low value of the interstellar polarization implied by the spectropolarimetry (L01). One argument for higher extinction, however, comes from the strength of the Na I D $(\lambda \lambda 5890,5896)$ interstellar absorption lines. From the echelle spectrum taken 4 days after discovery (Figure 14; a detailed analysis of the $\mathrm{Na} I \mathrm{D}$ interstellar lines will be the subject of a future paper), we measure $W_{\lambda}(\mathrm{D} 2 ; \lambda 5890)=0.86$ $\AA$ and $W_{\lambda}(\mathrm{D} 1 ; \lambda 5896)=0.65 \AA$, for a total equivalent width of $W_{\lambda}(\mathrm{Na}$ I D) $=1.51 \AA$ (cf. Jha et al. 1999). If we adopt the rough correlation derived by Barbon et al. (1990) between $W_{\lambda}$ (Na I D) and reddening,

$$
E(B-V)=0.25 W_{\lambda}(\mathrm{Na} \mathrm{I} \mathrm{D),}
$$

we would predict $E(B-V) \approx 0.38 \mathrm{mag}$ for SN $1999 \mathrm{em}$, significantly greater than the extinction implied by the other methods. The relation given by equation (14) is known to have very large scatter (likely due to variations in the dust-to-gas ratios among galaxies as well as the marginal ability of $\mathrm{Na}$ I D gas to serve as a tracer of the hydrogen gas column; saturation effects can also play a large roll; see, e.g., Munari \& Zwitter 1997; Issa, MacLaren, \& Wolfendale 1990), however, and we are inclined to favor the evidence supporting a lower total reddening; in $\S 4.1$ we shall also see that the EPM distances derived for $E(B-V)=0.38$ mag using the different filter combinations are very inconsistent with each other, further arguing against such high extinction. We therefore adopt the reddening preferred by Baron et al. (2000) of $E(B-V)=0.10 \mathrm{mag}$ and assign it an uncertainty of \pm 0.05 mag. The strength of the Na I D lines, however, does make us treat this value with some caution.

\subsection{The Photospheric Velocity and Color of SN 1999em During the Plateau}

From Figure 3 we see that the plateau phase of SN 1999em lasted until about 95 days after discovery, and our application of EPM will therefore be restricted to times somewhat earlier than the drop off the plateau. Figure 15 shows the velocity inferred from the flux minima of the first 3 hydrogen Balmer lines and Fe II $\lambda 5169$ during the first 130 days after discovery. As expected $(\S 1)$, the $\mathrm{H} \alpha$ and $\mathrm{H} \beta$ lines generally yield velocities significantly higher than the weaker $\mathrm{H} \gamma$ and Fe II $\lambda 5169$ lines. Curiously, after about day 40, the $\mathrm{H} \beta$ line yields velocities similar to, or even less than, those derived from Fe II $\lambda 5169$. In the day 76, 95, and 96 spectra the $\mathrm{H} \alpha$ absorption profile becomes corrupted by the excess emission described in $\S 2.2 .2$ (Figure 12), and the inferred velocity increases dramatically.

Traditionally, the Fe II $\lambda \lambda 4924,5018,5169$ line features have been used to estimate photospheric

velocity during the plateau phase of SNe II-P, with the occassional use of Sc II $\lambda 5527$ and Sc II $\lambda 5658$ as well. At very early times, before the iron lines become available, it has been customary to 


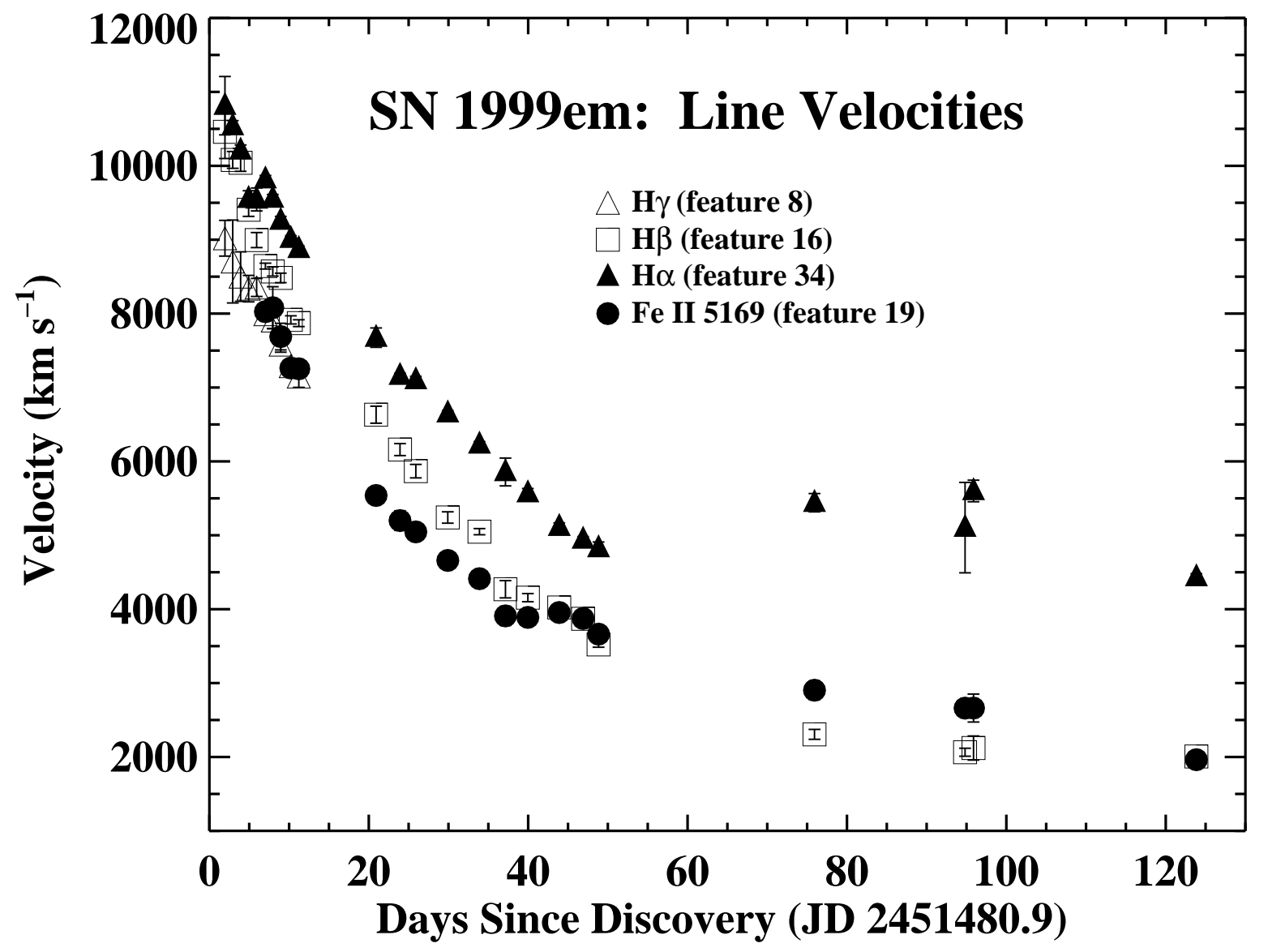

Fig. 15. - Line velocity determined from the P-Cygni absorption minima of 4 different features during the first 130 days after discovery of SN 1999em. 
either use the higher-order hydrogen Balmer lines (i.e., $\mathrm{H} \gamma$ and $\mathrm{H} \delta$ ) or else to scale the $\mathrm{H} \alpha$ and $\mathrm{H} \beta$ velocities using the ratio of their velocities to the iron features when the iron features first appear. Here, we shall follow two approaches. First, we derive velocities following the usual methods for determining photospheric velocity $\left(v_{\text {strong }}\right)$, relying mainly on the Fe II $\lambda \lambda 4924,5018,5169$ lines when they (or, at least, one of them) become available (day 7 onwards), and the higher-order hydrogen Balmer lines at earlier times. Second, we estimate photospheric velocity by using the weakest unblended features identified during each epoch $\left(v_{\text {weak }}\right)$. We shall then compare the EPM distance derived using each set of velocities.

Figure 16 shows the velocities measured for features observed during the first 11 days after discovery of SN 1999em. Through day $5, \mathrm{H} \alpha$ and $\mathrm{H} \beta$ yield similar velocities. He I $\lambda 5876$ and $\mathrm{H} \gamma$ yield similar velocities through day 6 , but significantly lower than those measured by $\mathrm{H} \alpha$ and $\mathrm{H} \beta$. From day 7 onwards, the velocities inferred from the weak He I $\lambda 5876$ and $\mathrm{H} \delta$ lines are somewhat lower than those measured using Fe II $\lambda 5169$ and $\mathrm{H} \gamma$. Our estimates of $v_{\text {strong }}$ and $v_{\text {weak }}$, then, will be the same for days 2 through 6 , but will differ from day 7 onwards. For days 2 through 6 , we take the weighted average of the He I $\lambda 5876$ and $\mathrm{H} \gamma$ velocities. From day 7 onwards, $v_{\text {strong }}$ is always determined by the available Fe II $\lambda \lambda 4924,5018,5169$ lines, and $v_{\text {weak }}$ is derived from the 4 weak, unblended features described in $\S 2.2 .3$ (features 12, 13, 21, and 22 in Figure 10).

Figure 17 shows the velocities derived from these features compared with those derived from the weighted average of the Fe II $\lambda \lambda 4924,5018$, 5169 lines from day 20 to day 100. Clearly, these weaker features yield systematically lower velocities than those derived from the stronger Fe II lines, and the difference appears to grow with time. To determine $v_{\text {weak }}$, then, we took the weighted mean of the available weak lines at each spectral epoch. Table 6 lists the $v_{\text {strong }}$ and $v_{\text {weak }}$ values adopted for each epoch; the reported uncertainty is the uncertainty of the weighted mean of the lines added in quadrature to the $1 \sigma$ spread in the velocities of the individual lines.

Figure 18 compares $v_{\text {strong }}$ and $v_{\text {weak }}$ during the plateau phase of SN 1999em. Since lower photospheric velocity estimates will result in a smaller derived distance (equation 3), it is useful to see whether such a velocity offset is ubiquitous among SNe II. Figure 19 shows the ratio of $v_{\text {weak }}$ to $v_{\text {strong }}$ as a function of time in the spectra of 6 other SNe II that had the high $\mathrm{S} / \mathrm{N}$ needed to make the measurements of these weak features. The good agreement of the data from other SNe II with SN 1999em suggests that EPM distances derived using the strong iron lines as photospheric velocity indicators will generally yield distances $5-10 \%$ larger than those using the weaker lines.

In order to derive an EPM distance to SN $1999 \mathrm{em}$, we need its $B V I$ magnitude at each of the spectral epochs. The spectral observations of SN 1999em listed in Table 3 are not, in general, coincident with the BVI photometric observations listed in Table 2. We therefore used a linear interpolation from the nearest photometric observations before and after the spectral observations. The reported photometric uncertainty represents the quadrature sum of the uncertainty in the interpolation (i.e., uncertainty in the location of the interpolated point due to the uncertainty in the slope of the line connecting the two photometric data points) with the photometric uncertainty 


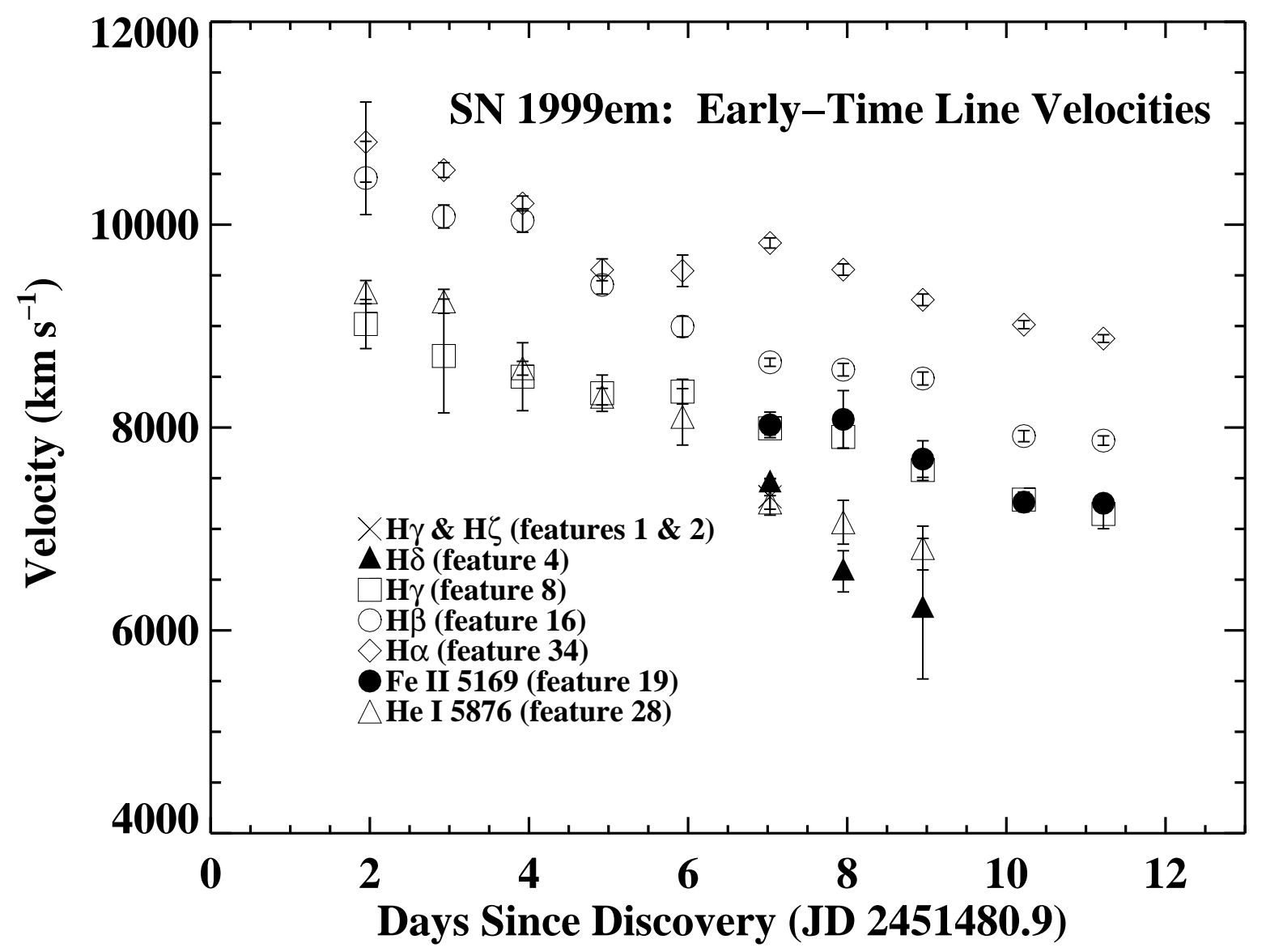

Fig. 16. - The velocity derived from absorption lines in the early-time spectrum of SN 1999em. Note that from day 7 onwards the velocity found for Fe II and $\mathrm{H} \gamma$ is somewhat higher than the weaker $\mathrm{H} \delta$ and He I $\lambda 5876$ lines suggest. 


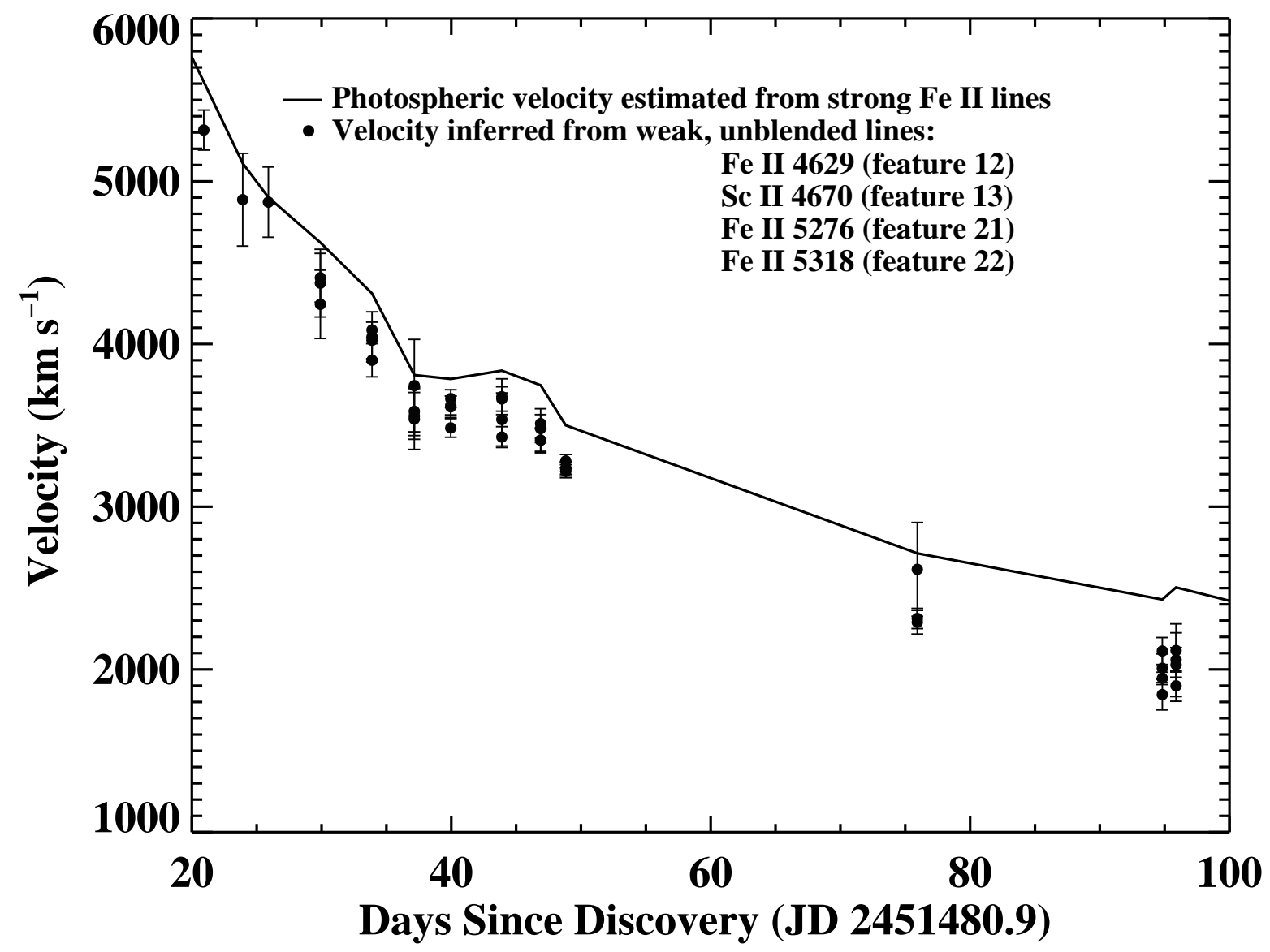

Fig. 17. - Temporal evolution of the velocity inferred from weak, unblended features compared with that derived from the stronger Fe II $\lambda \lambda 4924,5018,5169$ absorptions. The weaker features give consistently lower velocities. 
Table 6. Quantities Used in the EPM Analysis of SN 1999em

\begin{tabular}{lccccc}
\hline \hline & $v_{\text {strong }}{ }^{\mathrm{b}}$ & $v_{\text {weak }}{ }^{\mathrm{c}}$ & $B\left(\sigma_{B}\right)$ & $V\left(\sigma_{V}\right)$ & $I\left(\sigma_{I}\right)$ \\
Day & $\mathrm{km} \mathrm{s}^{-1}$ & $\mathrm{~km} \mathrm{~s}^{-1}$ & $\mathrm{mag}$ & $\mathrm{mag}$ & $\mathrm{mag}$ \\
\hline 1.9 & $9276(201)$ & $9276(201)$ & $13.8018(0.0271)$ & $13.8085(0.0241)$ & $13.5845(0.0287)$ \\
2.9 & $9221(192)$ & $9221(192)$ & $13.7951(0.0232)$ & $13.7887(0.0243)$ & $13.5620(0.0283)$ \\
3.9 & $8581(70)$ & $8581(70)$ & $13.8214(0.0311)$ & $13.7927(0.0201)$ & $13.5572(0.0261)$ \\
4.9 & $8310(77)$ & $8310(77)$ & $13.8434(0.0244)$ & $13.7877(0.0153)$ & $13.5411(0.0243)$ \\
5.9 & $8314(171)$ & $8314(171)$ & $13.8803(0.0286)$ & $13.8095(0.0176)$ & $13.5349(0.0292)$ \\
7.0 & $8026(178)$ & $7372(110)$ & $13.9225(0.0250)$ & $13.8371(0.0140)$ & $13.5294(0.0290)$ \\
7.9 & $8080(402)$ & $6809(372)$ & $13.9486(0.0191)$ & $13.8398(0.0130)$ & $13.4977(0.0251)$ \\
8.9 & $7642(271)$ & $6759(317)$ & $13.9837(0.0151)$ & $13.8568(0.0231)$ & $13.5072(0.0211)$ \\
10.2 & $7221(155)$ & $\ldots$ & $14.0089(0.0463)$ & $13.8481(0.0404)$ & $13.4876(0.0435)$ \\
11.2 & $7215(94)$ & $\ldots$ & $14.0354(0.0559)$ & $13.8389(0.0428)$ & $13.4702(0.0404)$ \\
20.9 & $5611(115)$ & $5315(174)$ & $14.4667(0.0230)$ & $13.8579(0.0290)$ & $13.4054(0.0270)$ \\
23.9 & $5110(122)$ & $4887(403)$ & $14.5791(0.0262)$ & $13.8804(0.0323)$ & $13.3818(0.0309)$ \\
25.9 & $4902(169)$ & $4872(305)$ & $14.6532(0.0209)$ & $13.8953(0.0197)$ & $13.3663(0.0253)$ \\
29.9 & $4623(52)$ & $4358(134)$ & $14.7882(0.0371)$ & $13.9358(0.0250)$ & $13.3490(0.0350)$ \\
33.9 & $4310(79)$ & $4047(86)$ & $14.8673(0.0250)$ & $13.9272(0.0200)$ & $13.3118(0.0210)$ \\
37.1 & $3809(89)$ & $3581(107)$ & $14.9257(0.0220)$ & $13.9209(0.0222)$ & $13.2919(0.0275)$ \\
39.9 & $3785(86)$ & $3596(86)$ & $14.9741(0.0180)$ & $13.9298(0.0190)$ & $13.2845(0.0320)$ \\
43.9 & $3836(69)$ & $3550(140)$ & $15.0025(0.0467)$ & $13.9123(0.0369)$ & $13.2226(0.0500)$ \\
46.9 & $3747(103)$ & $3443(64)$ & $15.0541(0.0300)$ & $13.9270(0.0350)$ & $13.2200(0.0390)$ \\
48.8 & $3500(141)$ & $3242(34)$ & $15.0800(0.0344)$ & $13.9387(0.0281)$ & $13.2148(0.0362)$ \\
75.9 & $2714(164)$ & $2312(78)$ & $15.4031(0.0373)$ & $14.0396(0.0271)$ & $13.2176(0.0333)$ \\
94.8 & $2430(190)$ & $1965(87)$ & $15.7184(0.0227)$ & $14.2587(0.0224)$ & $13.3670(0.0328)$ \\
95.8 & $2504(170)$ & $2012(106)$ & $15.7386(0.0210)$ & $14.2770(0.0220)$ & $13.3795(0.0320)$ \\
\hline & & & & & \\
\hline
\end{tabular}

Note. - Although only data up through day 76 were included when deriving the EPM distance, we list the values of the measured parameters through the early part of the transition to the nebular phase for completeness.

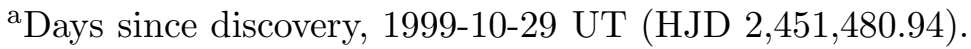

${ }^{\mathrm{b}}$ Velocity derived largely from Fe II $\lambda \lambda 4924,5018,5169$ lines.

${ }^{c}$ Velocity derived from weak, unblended lines; see text for details. 


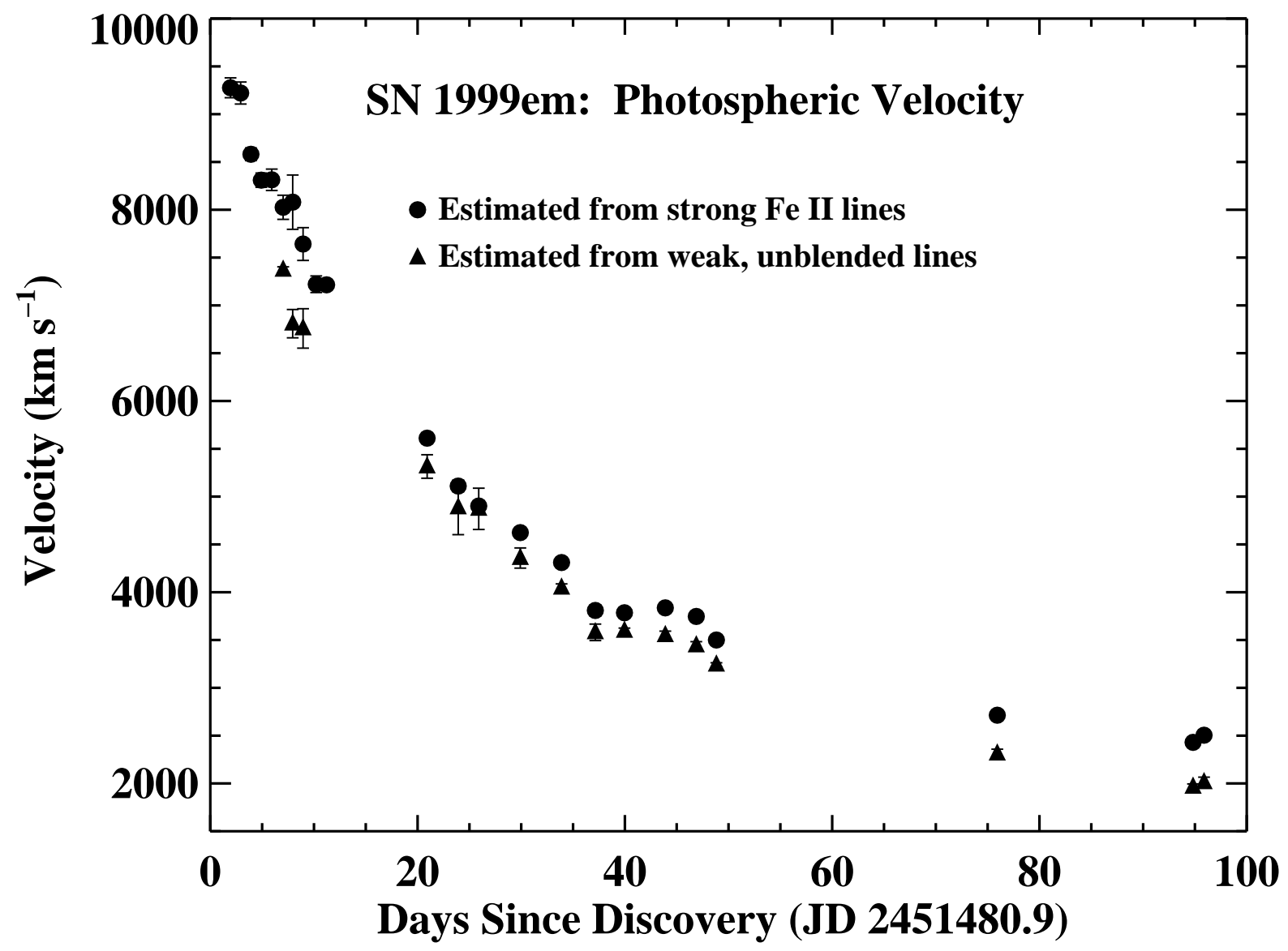

Fig. 18. - Comparison of photospheric velocity derived for SN 1999em by using the average of the strong Fe II $\lambda \lambda 4924,5018,5169$ lines $\left(v_{\text {strong }}\right)$ or the weaker unblended features $\left(v_{\text {weak }}\right)$ shown in Figure 17. 


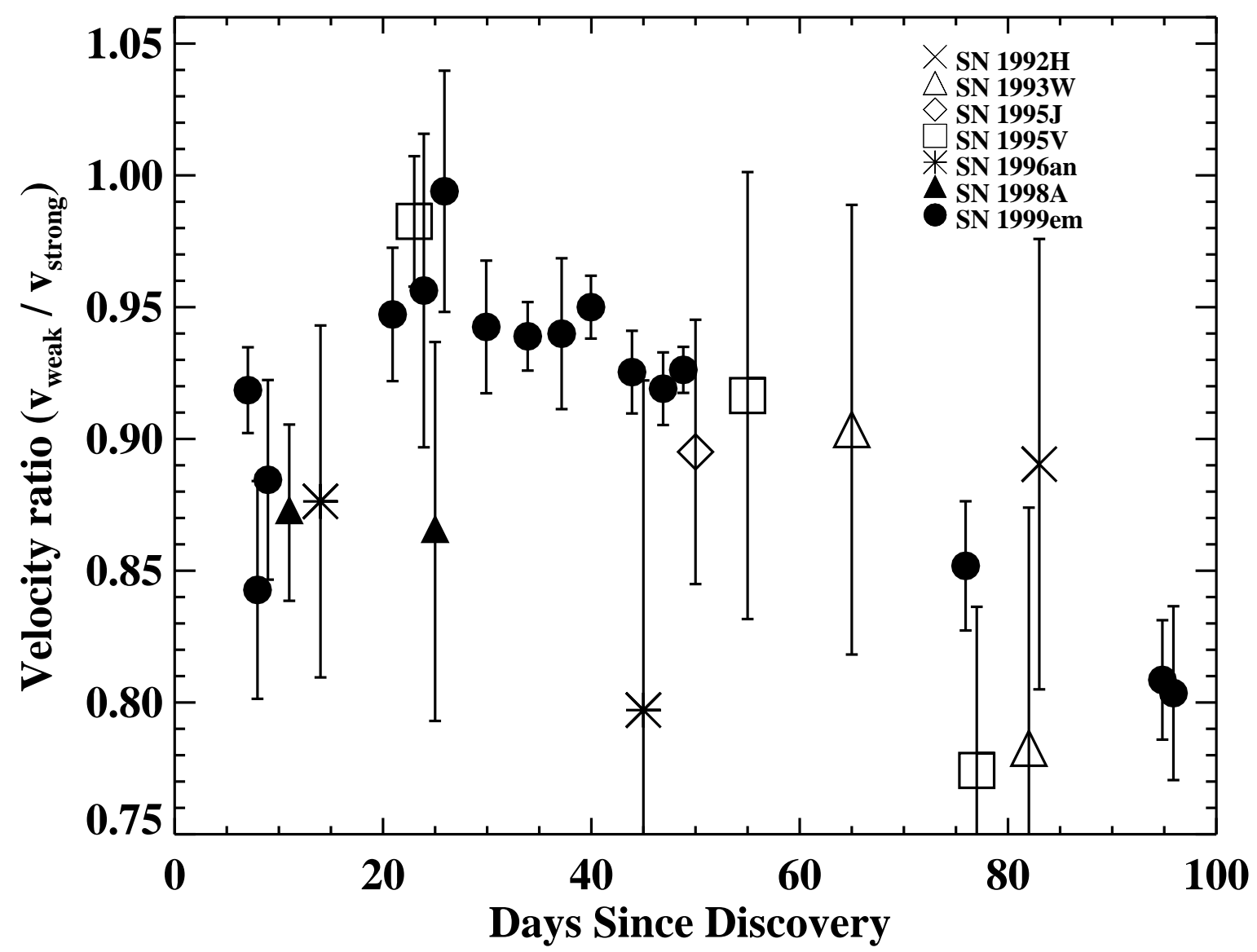

Fig. 19. - The temporal evolution of the ratio of the velocity derived from weak unblended lines to that derived from the strong Fe II $\lambda \lambda 4924,5018,5169$ features for 7 SNe II. Note that the relative phases of the $\mathrm{SNe}$ are somewhat uncertain since some may have been discovered significantly after explosion. 
of the nearest photometric point. The BVI magnitude and uncertainty adopted for SN 1999em at the times of spectral observations are given in Table 6 .

\section{The EPM Applied to SN 1999em}

Following the procedure outlined in $\S 1$, we derive the theoretical angular size $(\theta)$, flux dilution factor $(\zeta)$, and color temperature of SN $1999 \mathrm{em}$ for the three filter combinations $B V, B V I$, and $V I$, and list the results in Table 7. The uncertainty given for $\theta, \zeta$, and $T$ at each epoch is the $1 \sigma$ spread found for each parameter derived from 1000 simulated sets of photometry characterized by the photometric uncertainty listed in Table 6.

To determine the distance to SN 1999em for each filter combination we first need to decide how to weight the data, and then find the best-fitting line in the plot of $t$ vs. $\theta / v$ (equation [3]). Since the uncertainty of $\theta / v$ increases in direct proportion to the value of $\theta / v$, a weighting scheme based on the calculated uncertainty in each point is necessarily biased towards the earlier data. On the other hand, using the method of least squares with equal weighting is susceptible to undue influence by deviant points. We therefore opt for the more robust fitting method of using the criterion of the least absolute deviation for equally weighted data points (i.e., 'medfit' from Numerical Recipes; Press et al. 1992). Since SN $1999 \mathrm{em}$ begins to drop off the plateau near day 95 after discovery, we do not consider the data from day 95 onwards in the EPM analysis. The distances and times of explosion resulting from the 3 filter combinations and two photospheric velocity estimates are given in Table 8, along with uncertainties derived from 1000 simulated sets of data. Figures 20 through 25 show the relevant plots for each of the six distance estimates.

While the formal uncertainty of each derived distance listed in Table 8 is only a few percent, discrepancies among the distances derived using the dilution factors given for the various filter combinations run as high as $19 \%$. It is therefore clear that systematic, not statistical, error dominates the uncertainty in the EPM distance to SN $1999 \mathrm{em}$. In general, $D_{V I}>D_{B V I}>D_{B V}$. We note that the $50 \mathrm{~km} \mathrm{~s}^{-1}$ uncertainty in the redshift of SN 1999em (§ 2.2.3) contributes an uncertainty of only $\sim 2 \%$ to the derived distance. As expected, distances derived using the stronger iron features $\left(v_{\text {strong }}\right)$ are systematically about $10 \%$ larger than those derived using the weaker lines $\left(v_{\text {weak }}\right)$. For the weak lines, the (unweighted) average of the distances derived from the 3 filter combinations is $D_{\text {weak }}=8.24 \pm 0.56 \mathrm{Mpc}$, with $t_{o}=5.30 \pm 1.45$ days before discovery; the reported uncertainties are the standard deviations of the three individual data points from the mean value. ${ }^{22}$ Similarly, for the strong lines, $D_{\text {strong }}=9.03 \pm 0.78 \mathrm{Mpc}$ with $t_{o}=5.69 \pm 1.70$ days before discovery.

Since we believe that photospheric velocity is slightly overestimated by using the traditional Fe II $\lambda \lambda 4924,5018,5169$ lines, we conclude that the most accurate distance to SN $1999 \mathrm{em}$ is that

\footnotetext{
${ }^{22}$ We note that using the method of least squares to determine the best-fitting slope and intercept of the line described by equation 3 yields $D_{\text {weak }}=8.54 \pm 0.39 \mathrm{Mpc}$, with $t_{o}=6.20 \pm 0.92$ days before discovery.
} 
Table 7. Quantities Derived from the EPM Analysis of SN 1999em

\begin{tabular}{|c|c|c|c|c|c|c|c|c|c|}
\hline \multirow[b]{2}{*}{ Day $^{b}$} & \multicolumn{3}{|c|}{ Angular Size $\left(10^{8} \mathrm{~km} \mathrm{Mpc}^{-1}\right)^{\mathrm{a}}$} & \multicolumn{3}{|c|}{ Dilution Factor } & \multicolumn{3}{|c|}{ Temperature (K) } \\
\hline & $\theta_{B V}$ & $\theta_{B V I}$ & $\theta_{V I}$ & $\zeta_{B V}$ & $\zeta_{B V I}$ & $\zeta_{V I}$ & $T_{B V}$ & $T_{B V I}$ & $T_{V I}$ \\
\hline 1.9 & $6.13(1.01)$ & $7.57(0.50)$ & $8.46(0.70)$ & $0.411(0.020)$ & $0.429(0.005)$ & $0.444(0.005)$ & $17488(2121)$ & $14050(646)$ & 12150(798) \\
\hline 2.9 & $6.54(0.94)$ & $7.79(0.50)$ & $8.59(0.69)$ & $0.404(0.017)$ & $0.427(0.005)$ & $0.444(0.005)$ & $16809(1726)$ & $13834(625)$ & 12094(773) \\
\hline 3.9 & 7.14(1.09) & $8.12(0.51)$ & $8.76(0.66)$ & $0.394(0.016)$ & $0.424(0.005)$ & $0.443(0.004)$ & $15780(1739)$ & 13384(637) & $11915(701)$ \\
\hline 4.9 & $7.91(0.81)$ & $8.56(0.47)$ & $9.01(0.54)$ & $0.384(0.009)$ & $0.420(0.004)$ & $0.441(0.003)$ & $14709(1063)$ & $12882(524)$ & 11697(531) \\
\hline 5.9 & $8.25(0.97)$ & $8.99(0.50)$ & $9.50(0.68)$ & $0.380(0.010)$ & $0.417(0.003)$ & $0.439(0.003)$ & $14177(1192)$ & $12361(480)$ & $11185(614)$ \\
\hline 7.0 & $8.55(0.83)$ & $9.45(0.48)$ & $10.07(0.67)$ & $0.376(0.007)$ & $0.414(0.002)$ & $0.437(0.002)$ & $13703(945)$ & $11829(416)$ & $10641(527)$ \\
\hline 7.9 & $9.18(0.67)$ & $10.12(0.37)$ & $10.78(0.56)$ & $0.371(0.004)$ & $0.412(0.001)$ & $0.436(0.001)$ & 13012(667) & $11250(282)$ & 10135(394) \\
\hline 8.9 & $9.59(0.82)$ & $10.31(0.31)$ & $10.85(0.58)$ & $0.369(0.003)$ & $0.412(0.000)$ & $0.436(0.001)$ & $12529(716)$ & $11019(229)$ & $10032(431)$ \\
\hline 10.2 & $10.52(1.70)$ & $10.81(0.73)$ & $11.12(1.13)$ & $0.367(0.007)$ & $0.412(0.001)$ & $0.436(0.002)$ & $11723(1481)$ & 10640(530) & $9886(823)$ \\
\hline 11.2 & $11.45(1.79)$ & $11.28(0.79)$ & $11.34(1.09)$ & $0.367(0.010)$ & $0.412(0.002)$ & $0.436(0.002)$ & $10990(1465)$ & 10294(605) & 9779 (781) er \\
\hline 20.9 & $16.46(0.38)$ & $15.00(0.31)$ & $13.00(0.76)$ & $0.598(0.040)$ & $0.467(0.008)$ & $0.440(0.004)$ & $6523(249)$ & $7612(151)$ & $8820(439)^{-1}$ \\
\hline 23.9 & $16.64(0.36)$ & $15.69(0.34)$ & $13.82(0.76)$ & $0.699(0.050)$ & $0.497(0.011)$ & $0.445(0.006)$ & $6005(215)$ & $7110(162)$ & $8375(403)$ \\
\hline 25.9 & $16.73(0.23)$ & $16.06(0.31)$ & $14.34(0.59)$ & $0.776(0.040)$ & $0.520(0.011)$ & $0.450(0.005)$ & $5706(141)$ & $6822(127)$ & $8108(278)$ \\
\hline 29.9 & $16.75(0.32)$ & $16.52(0.29)$ & $15.22(0.77)$ & $0.914(0.071)$ & $0.565(0.015)$ & $0.460(0.009)$ & $5286(184)$ & $6378(119)$ & $7649(327)$ \\
\hline 33.9 & $17.16(0.27)$ & $17.00(0.20)$ & $15.84(0.47)$ & $1.061(0.056)$ & $0.603(0.011)$ & $0.466(0.007)$ & 4948(114) & $6085(77)$ & $7442(201)$ \\
\hline 37.1 & $17.51(0.30)$ & $17.29(0.24)$ & $16.15(0.58)$ & $1.180(0.058)$ & $0.632(0.014)$ & $0.470(0.008)$ & $4725(100)$ & $5899(84)$ & $7348(235)$ \\
\hline 39.9 & $17.65(0.28)$ & $17.40(0.24)$ & $16.40(0.65)$ & $1.258(0.052)$ & $0.652(0.014)$ & $0.474(0.009)$ & $4598(81)$ & $5778(79)$ & $7238(248)$ \\
\hline 43.9 & $18.06(0.61)$ & $17.94(0.36)$ & $17.39(1.03)$ & $1.352(0.123)$ & $0.690(0.025)$ & $0.486(0.018)$ & $4460(172)$ & $5582(120)$ & 6957(378) \\
\hline 46.8 & $18.18(0.59)$ & $18.00(0.32)$ & $17.60(0.85)$ & $1.431(0.104)$ & $0.714(0.023)$ & $0.491(0.016)$ & $4354(132)$ & $5472(100)$ & $6854(319)$ \\
\hline 48.8 & $18.18(0.52)$ & $18.04(0.29)$ & $17.82(0.76)$ & $1.462(0.101)$ & $0.728(0.020)$ & $0.496(0.015)$ & $4315(125)$ & $5410(86)$ & $6756(271)$ \\
\hline 75.9 & $19.47(0.79)$ & $18.14(0.23)$ & $18.72(0.62)$ & $2.002(0.120)$ & $0.890(0.030)$ & $0.531(0.017)$ & $3789(93)$ & $4857(82)$ & $6242(207)$ \\
\hline 94.8 & $18.94(0.67)$ & $16.94(0.22)$ & $17.99(0.51)$ & $2.259(0.086)$ & $0.988(0.025)$ & $0.560(0.018)$ & $3605(56)$ & $4609(57)$ & $5925(169)$ \\
\hline 95.8 & $18.82(0.65)$ & $16.84(0.22)$ & $17.93(0.51)$ & $2.264(0.083)$ & $0.994(0.022)$ & $0.562(0.018)$ & $3602(54)$ & $4596(49)$ & $5900(167)$ \\
\hline
\end{tabular}

Note. - Although only data up through day 76 were included when deriving the EPM distance, we list the values of the measured parameters through the early part of the transition to the nebular phase for completeness.

${ }^{a}$ Angular size of the optical photosphere (i.e., the surface of last scattering).

${ }^{\mathrm{b}}$ Days since discovery, 1999 October 29 UT (HJD 2,451,480.94). 
Table 8. EPM Distances to SN 1999em

\begin{tabular}{lcc}
\hline \hline Method $^{\mathrm{a}}$ & $\begin{array}{c}\text { Derived Distance } \\
(\mathrm{Mpc})\end{array}$ & $\begin{array}{c}\text { Time of Explosion } \\
\mathrm{b}\end{array}$ \\
\hline & & $(\mathrm{d})$ \\
$B V_{\text {weak }}$ & $7.68 \pm 0.24$ & $-3.70 \pm 0.72$ \\
$B V I_{\text {weak }}$ & $8.27 \pm 0.18$ & $-5.69 \pm 0.50$ \\
$V I_{\text {weak }}$ & $8.79 \pm 0.28$ & $-6.51 \pm 0.77$ \\
$B V_{\text {strong }}$ & $8.18 \pm 0.41$ & $-3.83 \pm 1.15$ \\
$B V I_{\text {strong }}$ & $9.20 \pm 0.27$ & $-6.08 \pm 0.74$ \\
$V I_{\text {strong }}$ & $9.72 \pm 0.42$ & $-7.16 \pm 1.01$ \\
\hline
\end{tabular}

Note. - The EPM distance derived to SN 1999em using the flux dilution factors of Eastman, Schmidt, \& Kirshner (1996) as updated by Hamuy et al. (2001). Uncertainties are the $1 \sigma$ uncertainties resulting from 1000 simulated sets of data characterized by the uncertainties in $\theta$ and $v$ found in Tables 6 and 7 .

aThe broadband filter combination $(B V, B V I$, or $V I$ ) used to derive the photospheric temperature, and the absorption lines used to derive photospheric velocity, where "strong" indicates Fe II $\lambda \lambda 4924,5018,5169$ and "weak" indicates the weaker, unblended features discussed in the text.

${ }^{b}$ Days before discovery, 1999-10-29 UT (HJD $2,451,480.94)$. 


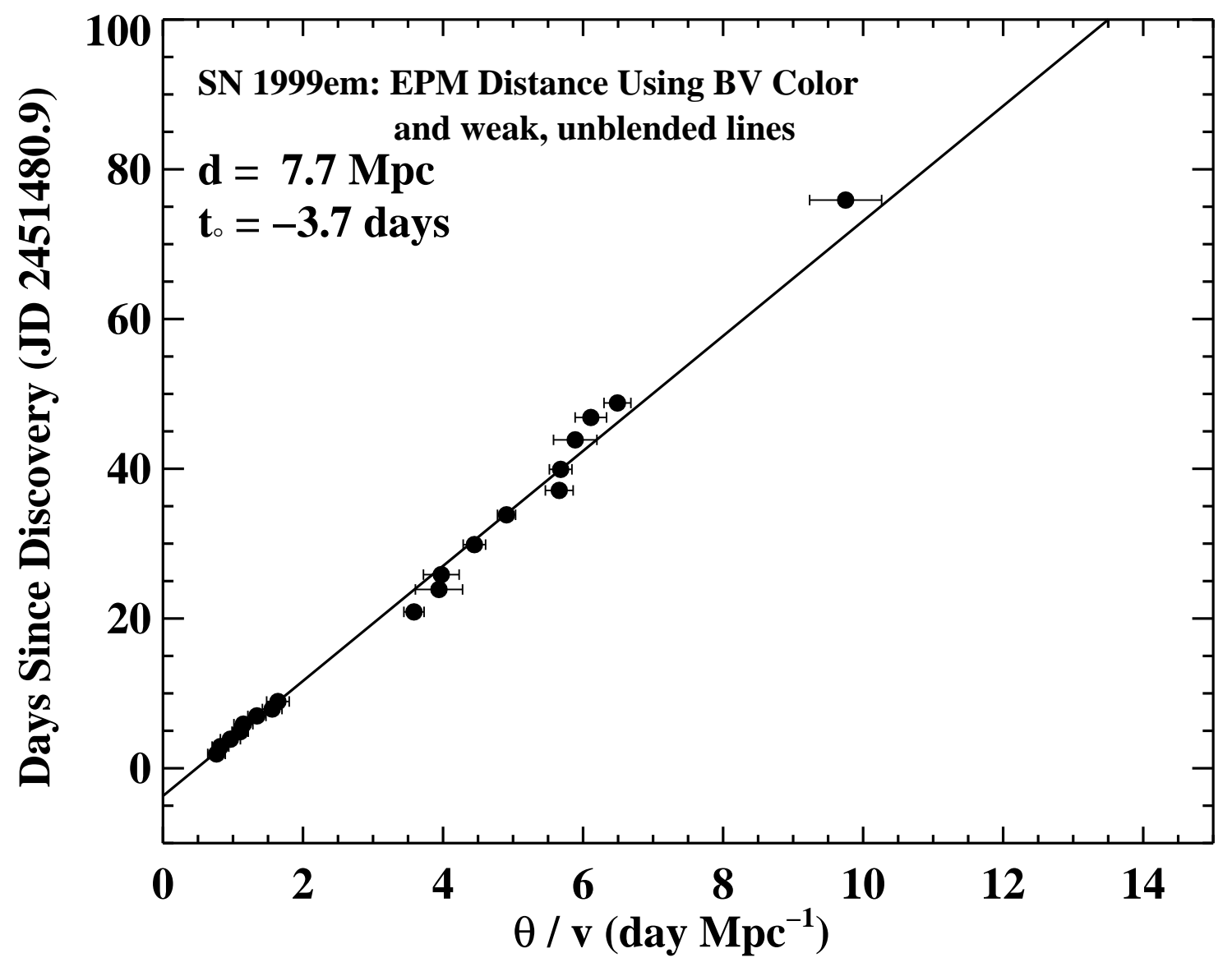

Fig. 20. - The parameters used to determine the distance to SN 1999em (equation [3]), with the line of best fit determined by using the criterion of least absolute deviations. The slope of this line yields the distance and the $y$-intercept the time of explosion of SN 1999em. Here, photospheric temperature is estimated using $B V$ photometry, and photospheric velocity is determined using $v_{\text {weak }}$, the velocity derived from weak, unblended features in the optical spectrum. 


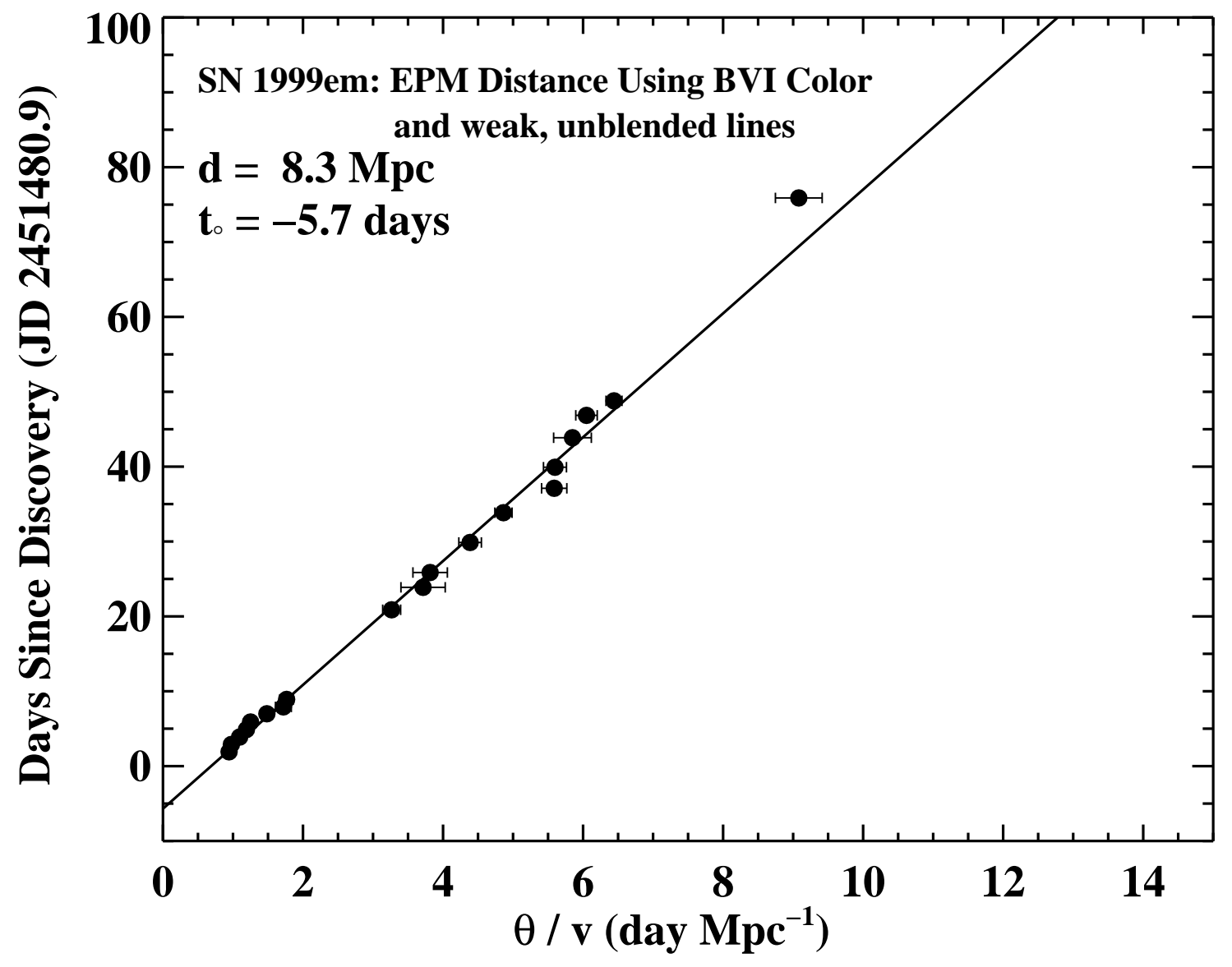

Fig. 21.- As in Figure 20, except using BVI photometry to determine photospheric color temperature. 


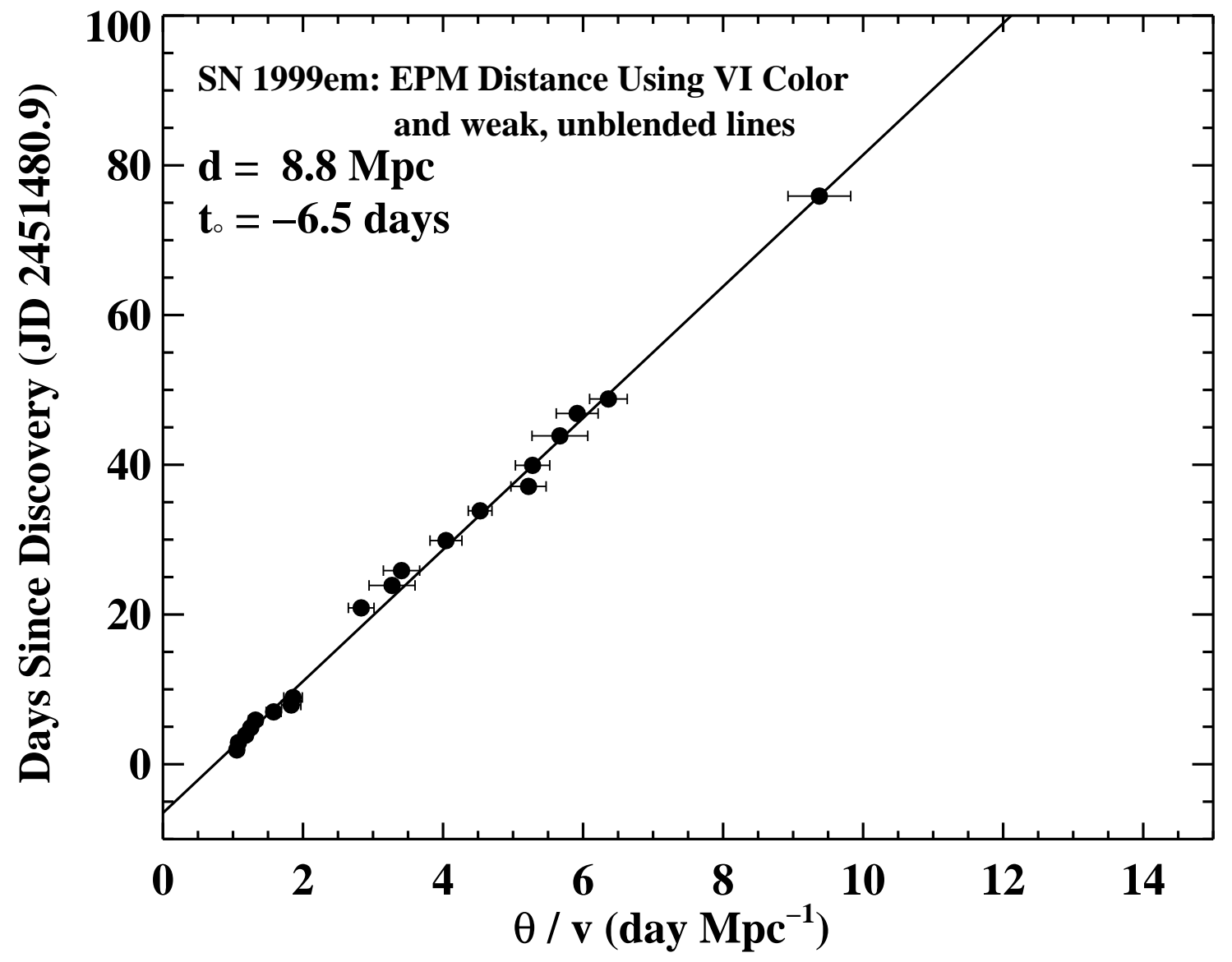

Fig. 22.- As in Figure 20, except using VI photometry to determine photospheric color temperature. 


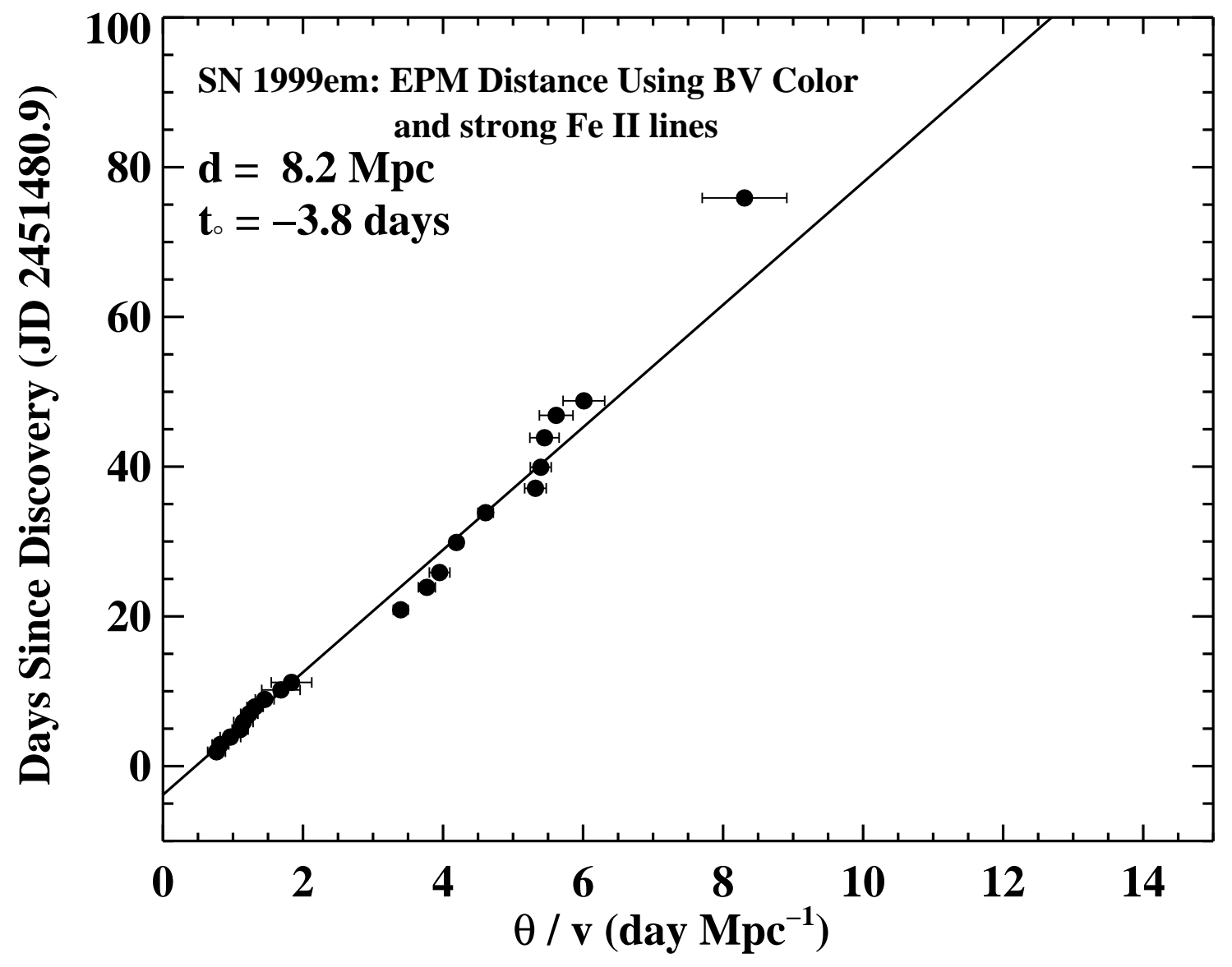

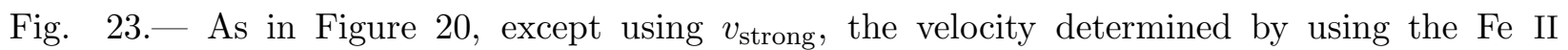
$\lambda \lambda 4924,5018,5169$ line features, to estimate photospheric velocity. 


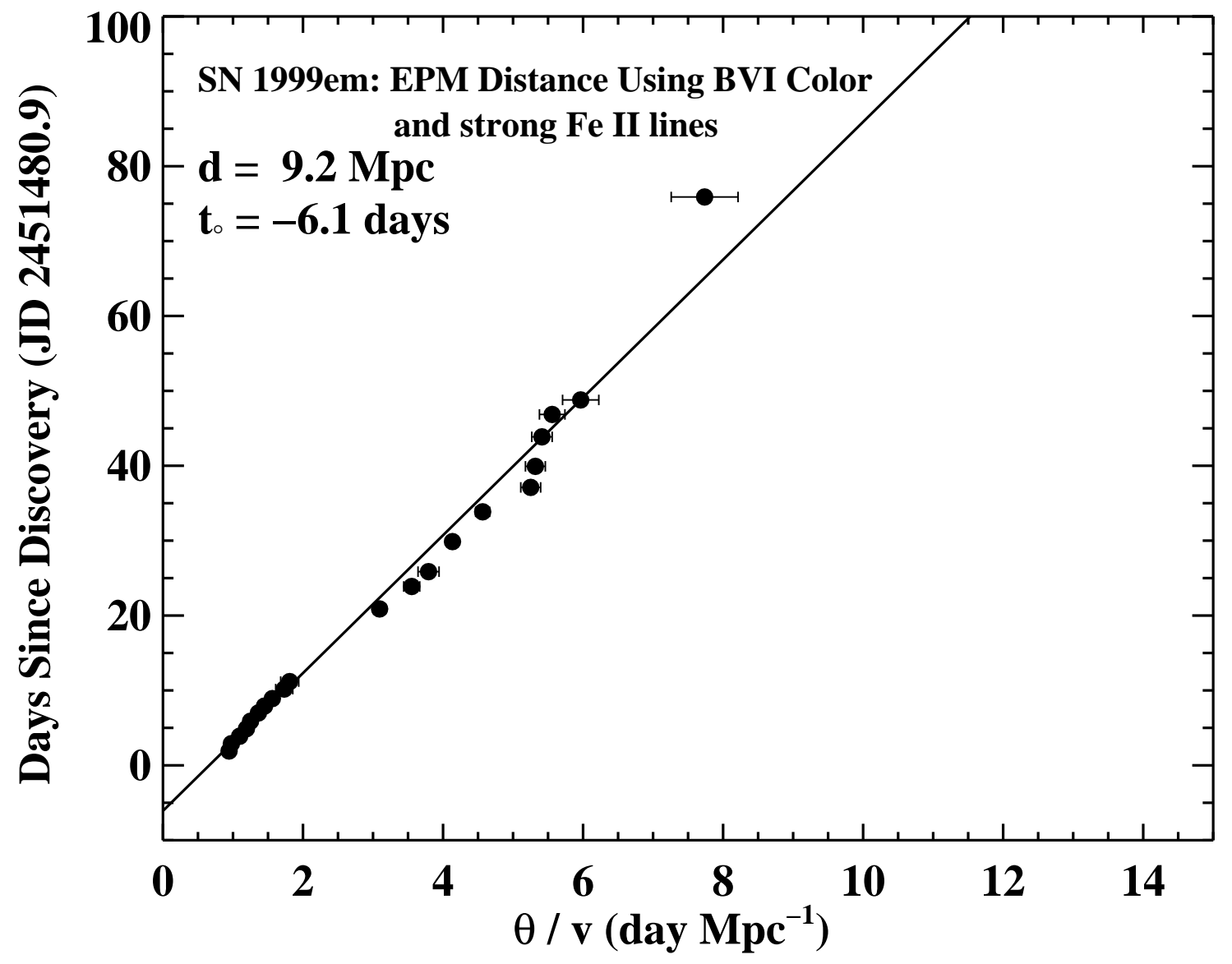

Fig. 24.- As in Figure 23, except using VI photometry to determine photospheric color temperature. 


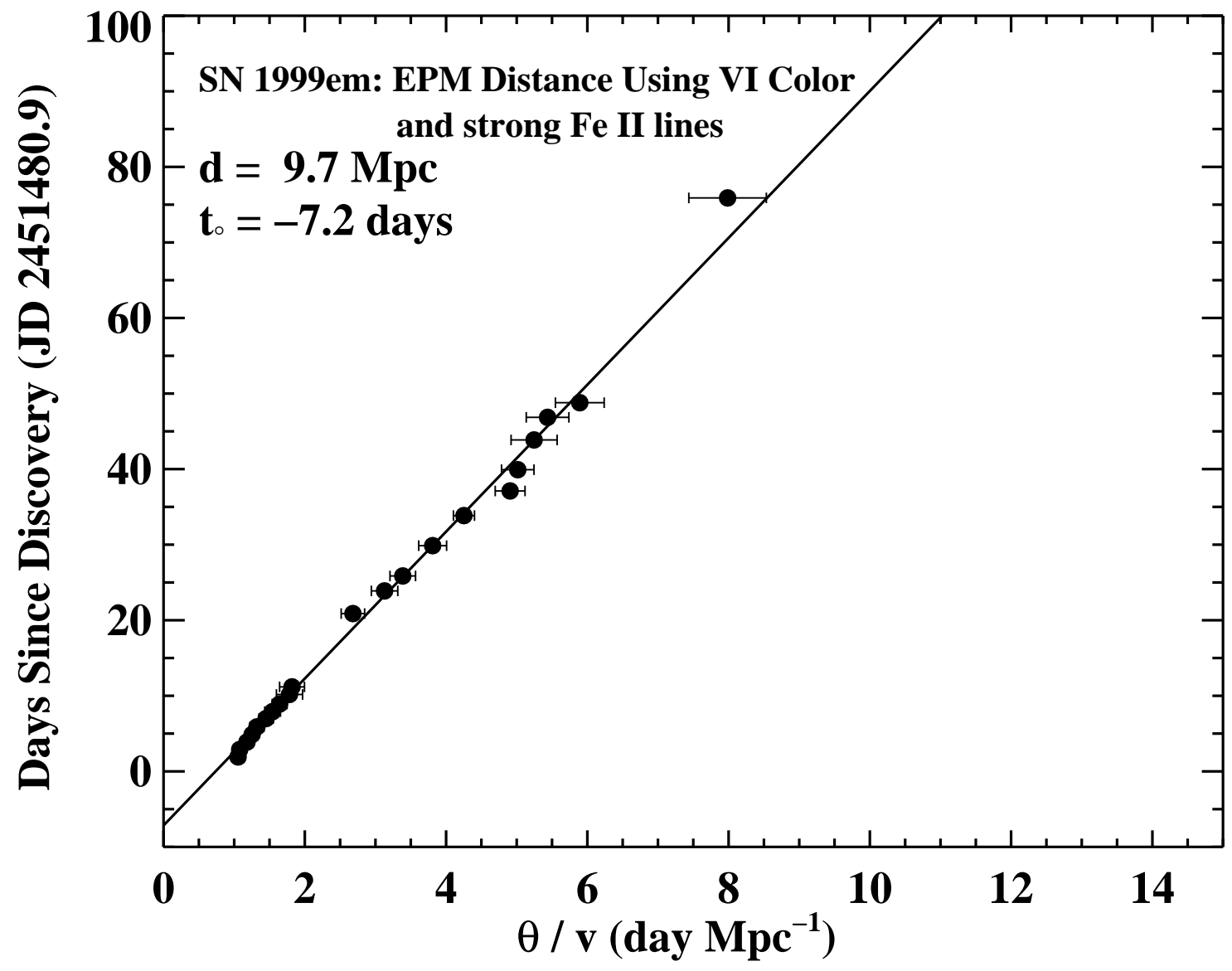

Fig. 25.- As in Figure 23, except using VI photometry to determine photospheric color temperature. 
found from the weak lines. Rounding to the nearest tenths place (in Mpc and days), then, yields $D=8.2 \pm 0.6 \mathrm{Mpc}$ with an explosion date of $5.3 \pm 1.4$ days prior to discovery as our best distance estimate.

\section{Discussion}

\subsection{Sources of Systematic Uncertainty}

Our preferred EPM distance to SN 1999em is in good agreement with the recent distance estimate to NGC 1637 of $D=7.8_{-0.9}^{+1.0} \mathrm{Mpc}$ found by Sohn \& Davidge (1998) using the galaxy's brightest supergiant stars as well as the EPM distance of $7.5 \pm 0.5 \mathrm{Mpc}$ recently reported by Hamuy et al. (2001) to SN 1999em. The major difference in the application of the EPM between our study and that of Hamuy et al. (2001) lies in the technique used to estimate photospheric velocity: while we rely on the velocity indicated by the flux minima of weak line features, Hamuy et al. cross-correlate the observed spectra with the model spectra of E96. A detailed discussion of the differences between these two methods is beyond the scope of the present study; we do note that the good agreement between the two resulting distances suggests that systematic differences between the two techniques may be small. It is also encouraging that two completely independent sets of data yield such consistent results.

From the scatter among the EPM distances derived using the 3 different filter combinations, it is clear that systematic effects dominate the uncertainty in the distance to this well-observed SN, and are at least of order $10 \%-20 \%$. One obvious potential source of systematic error is the uncertainty in the flux dilution factor, $\zeta$. The $5 \%-10 \%$ uncertainty in $\zeta$ (see $\S 1$ ) reported for the range of supernova atmospheres modeled by E96 translates directly to distance uncertainties of $5 \%-10 \%$. The maximum deviation of any atmosphere studied by E96 from the mean $\zeta$ is $24 \%$. If SN $1999 \mathrm{em}$ is such an extreme event and the degree of deviation between the true dilution factor and the average value varies among the different filter combinations studied, then the scatter among the derived distances may be explained.

A second source of systematic error is the uncertainty in the extinction assumed for SN 1999em, which affects each bandpass differently. In general, EPM-derived distances are fairly robust to uncertainty in extinction (E96; Schmidt et al. 1992), due to the approximate cancellation of terms in equation (5): since dust both reddens and diminishes the light received, both the measured flux, $f_{\nu}$, and the inferred blackbody flux, $B_{\nu}\left(T_{c}\right)$, decrease with increasing extinction. These two effects tend to cancel, producing a derived angular size, $\theta$, that does not vary much with changes in the reddening. An additional point to consider, however, is that $\zeta$ is also a strong function of $T_{c}$ for most filter combinations (generally decreasing with increasing $T_{c}$ ). The competition among these terms makes it difficult to generalize about how changes in reddening will alter the derived distance. For SN $1999 \mathrm{em}$, lowering $E(B-V)$ to 0.05 mag produces larger distances for all filter combinations $\left(D_{B V}=8.5 \mathrm{Mpc}, D_{B V I}=8.9 \mathrm{Mpc}\right.$, and $D_{V I}=9.0 \mathrm{Mpc}$; all distances are derived using $v_{\text {weak }}$ for 
the photospheric velocity $[\S 2.4]$, while raising the reddening to $E(B-V)=0.15$ mag lowers the derived distance for the $B V$ and $B V I$ filter combinations $\left(D_{B V}=7.0 \mathrm{Mpc}\right.$ and $\left.D_{B V I}=7.8 \mathrm{Mpc}\right)$ and keeps $D_{V I}$ at about the same distance $\left(D_{V I}=8.8 \mathrm{Mpc}\right)$. For $E(B-V)=0.05$ mag, taking the unweighted averages of the 3 filter combinations results in a distance and time of explosion of $D=8.8 \pm 0.2 \mathrm{Mpc}$ and $t_{o}=7.0 \pm 1.3$ days before discovery, while taking $E(B-V)=0.15$ mag yields $D=7.9 \pm 0.9 \mathrm{Mpc}$ and $t_{o}=3.7 \pm 1.6$ days before discovery. For the large extinction $(E[B-V]=0.38)$ suggested by the strength of the interstellar Na I D lines ( $\S 2.3)$, we note that $D_{B V}=4.7 \mathrm{Mpc}, D_{B V I}=6.2 \mathrm{Mpc}$, and $D_{V I}=10.3 \mathrm{Mpc}$. The large discrepancies among the three distances in the high-extinction case, along with very poor fits obtained in the $t$ vs. $\theta / v$ graphs (i.e., the points show large, systematic trends, and predict an explosion date somewhat after discovery) and unrealistically high temperatures derived at early times $\left(T_{\mathrm{BV}}>10^{6} \mathrm{~K}\right)$, all argue strongly against such a high reddening. While the lower extinction distances are much more self-consistent, we do note that no value of the extinction is found to make $D_{B V}=D_{B V I}=D_{V I}$.

In addition to the uncertainty due to the dilution factor and extinction, asphericity could certainly contribute as well. However, it is not easy to see how asphericity could affect the individual bandpasses differently, to produce the filter-dependent discrepancies that are seen. L01 found that the polarization of SN 1999em increased with time, perhaps suggesting an increase in asphericity deeper into the ejecta. Barring a special viewing angle, increasing asphericity should produce a changing EPM distance with time. However, as shown in Figure 26, the derived distance to SN $1999 \mathrm{em}$ stays reasonably stable for all 3 filter combinations. Unless offsetting effects exist, the lack of obvious and consistent temporal trends across the three filter sets argues against the idea that the degree of asphericity increases with increasing depth in the SN ejecta. We therefore conclude that the most likely sources of systematic error are the uncertainty in the flux dilution factors and the extinction, and that these uncertainties clearly dominate over the statistical uncertainty resulting from the application of the EPM in any single filter combination alone. It should be noted that the uncertainty given for our preferred distance, $D=8.2 \pm 0.6 \mathrm{Mpc}$, incorporates only the evident systematic uncertainty resulting from the application of the EPM with the three different filter combinations, and includes neither the uncertainty in extinction nor the disagreements among different groups on the overall level of the dilution factor (e.g., Baron et al. 1995).

\subsection{Comparison between EPM and Cepheid Distances}

Cepheid variables remain the most widely used and well studied extragalactic primary distance indicators. In particular, more than two dozen galaxies now have Cepheid distances obtained with the Hubble Space Telescope (HST) by the Distance Scale Key Project (e.g., Ferrarese et al. 2000; Freedman et al. 2001) and other groups (e.g., Saha et al. 1999; Tanvir, Ferguson, \& Shanks 1999). Observing strategies and reduction techniques are well defined (e.g., Tanvir 1999). The slope and form of the Cepheid period-luminosity (PL) relation is quite well established from observations of LMC variables (e.g., Udalski et al. 1999). However, there is still some controversy over the distance 


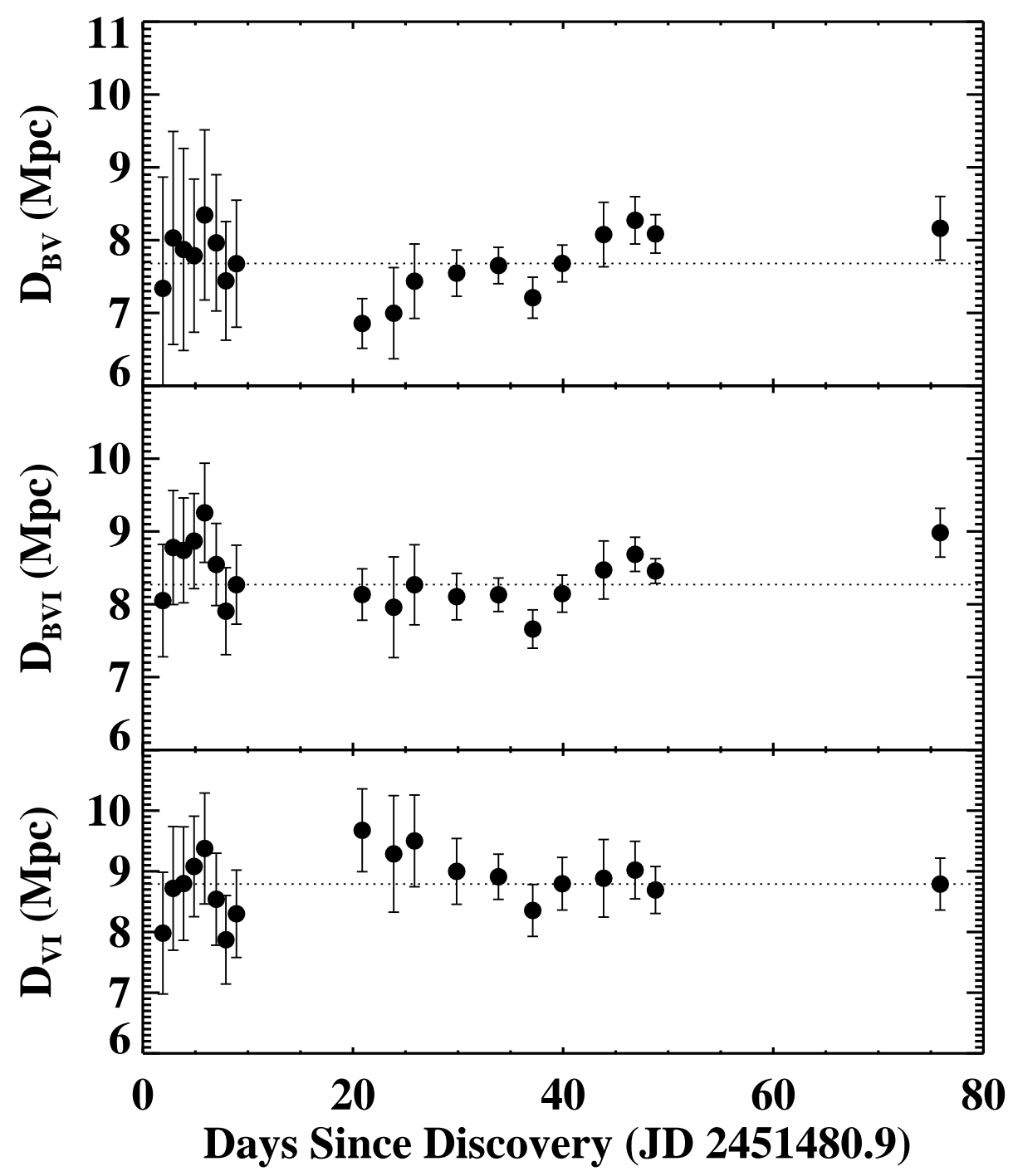

Fig. 26. - The derived EPM distance to SN $1999 \mathrm{em}$ as a function of time for the $B V$, $B V I$, and $V I$ filter sets, with weak, unblended features used to estimate photospheric velocity. 
to the LMC itself, which sets the PL zero-point (e.g., Silbermann et al. 1999). Considering all sources of error, the Key Project has recently announced "final" results for the Cepheid distances, with quoted random uncertainties of about $5 \%$ for each distance; systematic uncertainty, incorporating uncertainty introduced by the LMC zero-point offset, metallicity correction, photometric zero-point, and aperture correction is at the $10 \%$ level (Freedman et al. 2001).

Including the most recent additions, Cepheid distances now exist to 1 galaxy that hosted a SN II-P (SN 1973R), 5 galaxies that hosted photometrically or spectroscopically peculiar SNe II (SN 1970G, SN 1979C, SN 1987A, SN 1989L, and SN 1993J), and 3 galaxies in the same group in which a SN II-P occurred (SN 1968L, SN 1969L, and SN 1988A). Given the peculiar nature of some of these SNe and the uncertainties involved in comparing distances derived to different galaxies within the same group, it is quite impressive that E96 found $D_{\text {Cepheids }} / D_{\text {EPM }}=0.98 \pm 0.08$, based on the distances to 6 galaxies (or galaxies in the same group) that had Cepheid distances at the time of their study. Table 9 lists the Cepheid distances that were used by E96, the most recent Cepheid distances (taken from Freedman et al. 2001), and the EPM distances to 8 galaxies; we do not include SN 1993J in M81 since detailed models of its (likely unusual) early-time dilution factor evolution are needed to produce an EPM distance truely independent of the Cepheid scale (see Clocchiatti et al. 1995).

Due to the recent modifications of the zero-point offset and refinement of the Cepheid distance technique itself, it is seen that the Cepheid distances to these galaxies have changed somewhat; in fact, nearly all of the Cepheid distances have decreased from their previous values. Using the revised Cepheid distances for the 6 SNe that were compared by E96, and using the same comparison technique (i.e., finding the simple weighted mean and $1 \sigma$ uncertainty in the weighted mean making no special consideration for the added uncertainty associated with comparing Cepheid distances to galaxies in the same group as a galaxy that hosted a $\mathrm{SN}$ II), now yields $D_{\text {Cepheids }} / D_{\mathrm{EPM}}=$ $0.88 \pm 0.07$; including all 8 available galaxies yields $D_{\text {Cepheids }} / D_{\mathrm{EPM}}=0.87 \pm 0.06$. Of course, comparing group-member galaxy distances is a risky business and certainly increases the actual uncertainty of the comparison. If we eliminate the three group member comparisons (i.e., SN 1968L, SN 1969L, and SN 1988A), we then derive $D_{\text {Cepheids }} / D_{\mathrm{EPM}}=0.96 \pm 0.09$. Clearly, additional direct comparisons, particularly to spectroscopically normal SNe II-P, are needed to produce a more statistically meaningful comparison. Despite these being the "final" Cepheid distances, it should also be noted that other workers find evidence for a significantly longer Cepheid distance scale (e.g., Parodi et al. 2000), and that even within the Key Project itself longer distance scales have been given (e.g., Ferrarese et al. 2000).

Nonetheless, the "best" Cepheid distances currently available suggest that EPM distances may be systematically too high. Since systematic uncertainty clearly remains at at least the $10 \%$ level for Cepheids, and at the 10-20\% level for EPM, it is not clear how significant the observed discrepancy actually is. From our study of the effects that using strong lines to estimate photospheric velocity has on the derived distance, we might conclude that some of the difference between the EPM and Cepheid scales is due to inaccuracies in the photospheric velocity estimates; indeed, the distances 
Table 9. EPM and Cepheid Distances to Type II Supernovae

\begin{tabular}{|c|c|c|c|c|c|}
\hline \multirow[t]{2}{*}{ Supernova } & \multicolumn{2}{|c|}{ Galaxy Name } & \multicolumn{2}{|c|}{ Cepheid Distance } & \multirow{2}{*}{$\begin{array}{c}\text { EPM Distance } \\
(\mathrm{Mpc})\end{array}$} \\
\hline & SN host & Cepheid galaxy & $\mathrm{D}_{\text {old }}(\mathrm{Mpc})^{\mathrm{a}}$ & $\mathrm{D}_{\text {new }}(\mathrm{Mpc})^{\mathrm{b}}$ & \\
\hline SN 1968L & NGC 5236 & NGC 5253 group & $4.1 \pm 0.4$ & $3.15 \pm 0.20$ & $4.5_{-0.8}^{+0.7}$ \\
\hline SN 1969L & NGC 1058 & NGC 925 group & $9.29 \pm 0.69$ & $9.16 \pm 0.17$ & $10.6_{-1.1}^{+1.9}$ \\
\hline SN 1970G & NGC 5457 (M101) & NGC 5457 (M101) & $7.5 \pm 0.8$ & $6.70 \pm 0.34$ & $7.4_{-1.5}^{+1.0}$ \\
\hline SN 1973R & NGC 3627 & NGC 3627 & $\cdots$ & $10.05 \pm 0.37$ & $15 \pm 7$ \\
\hline SN 1979C & NGC 4321 (M100) & NGC 4321 (M100) & $17.1 \pm 1.8$ & $15.21 \pm 0.49$ & $15 \pm 4$ \\
\hline SN 1987A & LMC & $\mathrm{LMC}$ & $0.051 \pm 0.003$ & $0.050 \pm 0.002$ & $0.049 \pm 0.006$ \\
\hline SN 1988A & NGC 4579 & Virgo cluster & $17.1 \pm 1.8$ & $15.28 \pm 0.35$ & $20 \pm 3$ \\
\hline SN 1989L & NGC 7331 & NGC 7331 & $\ldots$ & $14.72 \pm 0.61$ & $17 \pm 4$ \\
\hline
\end{tabular}

${ }^{a}$ Cepheid distance used by Eastman, Schmidt, \& Kirshner (1996).

${ }^{b}$ Metallicity-corrected Cepheid distance and random uncertainty reported by Freedman et al. (2001); systematic uncertainty in the Cepheid distance scale is $\sim 10 \%$.

${ }^{\mathrm{c}}$ Distances and uncertainties reported by Eastman, Schmidt, \& Kirshner (1996). 
derived by using the strong Fe II $\lambda \lambda 4924,5018,5169$ lines would tend to produce EPM distances systmatically $5-10 \%$ too large. However, since it is not certain exactly which lines were used in each of the previous EPM studies to estimate photospheric velocity (and we note that using the Sc II $\lambda 5658$ line with $\lambda_{\circ}=5657.9 \AA$ will produce velocities that are lower than those found from the weak, unblended lines [§ 2.2.3]), it is difficult to generalize.

\subsection{SNe II-P as Standard Candles}

To obtain the EPM distance to a SN II-P requires a well-spaced series of high-quality spectral and photometric data, and even with an excellent data set distance uncertainties of $10 \%-20 \%$ currently still remain. As we look toward pushing EPM out to higher redshifts, it is clear that the most demanding observational requirement is the need to obtain multiple spectral epochs in order to determine the photospheric velocity. This is markedly different from the case for SNe Ia, which require only a single spectral epoch near (or, preferably, before) maximum light to identify it (e.g., Coil et al. 2000). An interesting suggestion has been made by Höflich et al. (2000) that distances accurate to $\sim 30 \%$ should be achievable by treating SNe II-P as standard candles. Based on theoretical models with a wide range of parameters (explosion energy, metallicity, mass loss of progenitor, etc.), Höflich et al. (2000) find that although the peak luminosity varies greatly among SNe II-P, the mean absolute brightness during the plateau phase is quite insensitive to the initial parameters, with $\bar{M}_{V}$ (plateau) $\approx-17.6 \pm 0.6$ mag. Treating SNe II-P as standard candles is particularly attractive since they have a unique light-curve shape, obviating the need for even a single spectrum. Only a few deep images, roughly every 50 days (in the SN rest frame), are needed to discover, identify, and measure the mean plateau brightness; there is no need to follow the SN after the plateau ends and the SN becomes faint.

To date, EPM distances have been derived to 10 SNe II-P, of which 8 have published $V$-band photometry sampling the plateau epoch. Adopting the EPM distance, the published photometry, and the extinction used in the EPM analysis (in some cases modified slightly due to the more accurate reddening values provided by the recent dust maps of Schlegel et al. [1998]), we derive the average $V$-band magnitude during the plateau for these 8 SNe II-P, and list the results in Table 10 along with SN $1999 \mathrm{em}$. To estimate the average $V$-band magnitude during the plateau, we took the average of the $V$-band data between days 20 and 100 after the date of explosion (as determined by the EPM analysis). With the exception of SN 1973R, which is anomalously bright, ${ }^{23}$ all of the values cluster within 1.2 mag of each other. In fact, the weighted mean absolute $V$-band magnitude during the plateau epoch for SNe II-P, not including SN $1999 \mathrm{em}$, is $\bar{M}_{V}$ (plateau) $=-16.8_{-0.5}^{+0.4}$ mag, where the reported uncertainty is the weighted standard deviation of the data from the mean. Including

\footnotetext{
${ }^{23}$ Note that the recent Cepheid distance to this galaxy is significantly less than that estimated by EPM (Table 9). If the Cepheid distance is correct, then SN 1973R actually has $\bar{M}_{V}$ (plateau) $=-18.2$ mag, making it somewhat more consistent with the other SNe II-P.
} 
Table 10. Average $V$-Band Magnitude of SNe II-P During the Plateau Phase

\begin{tabular}{lcccccc}
\hline \hline Supernova & $\begin{array}{c}A_{V}{ }^{\mathrm{a}} \\
(\mathrm{mag})\end{array}$ & $\begin{array}{c}\bar{m}_{V}{ }^{\mathrm{b}} \\
(\mathrm{mag})\end{array}$ & $\begin{array}{c}\text { Distance } \\
(\mathrm{Mpc})\end{array}$ & $\begin{array}{c}\bar{M}_{V}^{\mathrm{c}} \\
(\mathrm{mag})\end{array}$ & \multicolumn{2}{c}{ References } \\
\cline { 6 - 7 } SN 1968L & 0.03 & 12.0 & $4.5_{-0.8}^{+0.7}$ & $-16.3_{-0.3}^{+0.3}$ & 1 & 2 \\
SN 1969L & 0.18 & 13.2 & $10.6_{-1.1}^{+1.9}$ & $-16.9_{-0.4}^{+0.2}$ & 1 & 2 \\
SN 1973R & 2.80 & 11.8 & $15_{-7}^{+7}$ & $-19.1_{-1.0}^{+1.0}$ & 1 & 2 \\
SN 1988A & 0.15 & 14.6 & $20_{-3}^{+3}$ & $-16.9_{-0.3}^{+0.3}$ & 1 & 2 \\
SN 1990E & 1.5 & 14.2 & $18_{-2}^{+3}$ & $-17.1_{-0.4}^{+0.2}$ & 3 & 2 \\
SN 1990ae & 0.5 & 19.3 & $115_{-25}^{+35}$ & $-16.0_{-0.7}^{+0.5}$ & 1 & 2 \\
SN 1992H & 0.28 & 14.6 & $20.2_{-4}^{+4}$ & $-16.9_{-0.4}^{+0.4}$ & 4 & 2 \\
SN 1999em & 0.31 & 13.7 & $8.2_{-0.6}^{+0.6}$ & $-15.9_{-0.2}^{+0.2}$ & 6 & 2 \\
\hline
\end{tabular}

Note. - All uncertainties are $1 \sigma$.

${ }^{a}$ Estimated total extinction in the $V$ band. If the reference from which the distance estimate was taken also included a derivation of the total extinction of the SN based on properties of the SN, then that value was used. In cases where the extinction was estimated for the Galaxy and host individually, and then summed, we have updated the Galactic extinction to be the values provided by the dust maps of Schlegel et al. (1998), replacing those given by Burstein \& Heiles (1982).

${ }^{\mathrm{b}}$ Average apparent $V$-band magnitude during the plateau phase, derived as the average of all $V$-band data between days 20 and 100 after explosion, corrected for extinction.

${ }^{\mathrm{c}}$ Average absolute $V$-band magnitude during the plateau phase, with uncertainty derived solely from the uncertainty in the EPM distance estimate.

dPhotometry data taken from Schmidt et al. (1992), and references therein.

References. - (1) Schmidt et al. 1992, and references therein; (2) Schmidt et al. 1994a; (3) Schmidt et al. 1993; (4) Clocchiatti et al. 1996; (5) Schmidt et al. 1994b; (6) This work. 
SN $1999 \mathrm{em}$ lowers the average to $\bar{M}_{V}$ (plateau) $=-16.4_{-0.6}^{+0.6} \mathrm{mag}$. The data thus favor a somewhat fainter mean plateau $V$-band brightness than the models of Höflich et al. (2000) predict, but with a similar spread around the mean, much of which may be due to the uncertainty in the EPM technique itself. From just these 9 SNe II-P, we would conclude that distances to SNe II-P accurate to $\pm 28 \%(1 \sigma)$ could be achieved with the standard-candle assumption, which compares well with the current level of systematic uncertainty in EPM, and is much less observationally taxing. Clearly, additional distances to SNe II-P are needed to better quantify the uncertainty resulting from the standard-candle assumption. Although the use of SNe II-P as standard candles will not achieve the same individual accuracy as SNe Ia, the removal of the need to obtain a spectrum, coupled with their certain existence at high redshift (before SNe Ia even occur, perhaps), may yet make them the best SN class for cosmology at redshifts beyond 2.0.

\subsection{Is SN 1999em a "Typical" SN II-P?}

In $\S 2.3$ we raised the possibility that the $B-V$ color evolution of SN 1999em might be somewhat different from the normal evolution of SNe II-P during the plateau phase. In $\S 4.3$ we found that SN 1999em was somewhat fainter during the plateau than other SNe II-P with EPM distances; at our derived distance, SN 1999em also reached a peak extinction-corrected $B$-band magnitude of only $M_{B}=-16.2 \pm 0.2 \mathrm{mag}$, which places it on the fainter end of the range observed for SNe II-P (Patat et al. 1994; Miller \& Branch 1990). This naturally leads to the question: is SN 1999em unusual? If so, could this have affected the EPM analysis? More to the point, are the observed properties of SN $1999 \mathrm{em}$ similar to the theoretical models used to derive the dilution factors $(\zeta)$ by $\mathrm{E} 96$ ?

Since SN 1999em evolved more rapidly toward red colors than the prototypical Type II-P SN 1969L (see Figure 13), we first consider the temporal evolution of its color temperature. The lowest color temperatures for any of the models studied by E96 are those for model s15.60.1, which has $T_{B V}=4132 \mathrm{~K}$ and $T_{B V I}=5418 \mathrm{~K}$, and $10 \mathrm{~h} .60 .1$, which has $T_{V I}=6638 \mathrm{~K} ;{ }^{24}$ only 1 other model studied (p6.40.1) has $T_{B V}<5000 \mathrm{~K}$ and $T_{B V I}<6000 \mathrm{~K}$. However, a quick look at Figure 5 and Table 7 reveals that SN $1999 \mathrm{em}$ drops below $T_{B V}=5000 \mathrm{~K}$ and $T_{B V I}=6000 \mathrm{~K}$ near day 30 , and below $T_{B V}=4132 \mathrm{~K}$ and $T_{B V I}=5418 \mathrm{~K}$ around day 50 . This means that after about day 30, the color temperature of SN $1999 \mathrm{em}$ is only represented by 2 of the 63 models studied by E96, and after day 50 it has no representation in the model set used to derive $\zeta\left(T_{c}\right)$, but rather relies on extrapolation. At these low temperatures, $\zeta_{B V}$ and $\zeta_{B V I}$ are changing very rapidly with temperature. A contribution to the distance uncertainty could thus come from the lack of models near the observed color temperature of SN 1999em during the late portion of the recombination

\footnotetext{
${ }^{24}$ We note that a small change in the model's color-temperatures will result from the use of the Hamuy et al. (2001) filter functions in place of those of E96; for the low-temperature regime of interest here, however, these differences should be minimal.
} 
phase. We note that increasing the reddening of SN 1999em to the upper limit given by Baron et al. (2000; see also $\S 2.3$ ) of $E(B-V)=0.15 \mathrm{mag}$ raises $T_{B V I}$ by $400 \mathrm{~K}$ and $T_{B V}$ by only about $200 \mathrm{~K}$ on day 30.

From its relative faintness and rapid color evolution during the plateau there is therefore some evidence that SN $1999 \mathrm{em}$ was a somewhat unusual II-P event. It is possible that the average $\zeta$ values derived by E96 do not adequately represent the evolution seen in SN 1999em, and may point toward the need for models specifically crafted for it to increase the accuracy of its distance.

One way to help place the theoretically derived flux dilution factor on a more sound empirical footing is to directly compare the EPM distance to NGC 1637 with that derived using other primary extragalactic distance indicators. At a distance of $8.2 \mathrm{Mpc}$, NGC 1637 makes an ideal target for a Cepheid study using HST: it is rather face-on $\left(i \approx 32^{\circ}\right)$, has low foreground extinction, and has a metallicity of $\log (\mathrm{O} / \mathrm{H})+12 \approx 9.1$ in the region near the SN (van Zee et al. 1998), typical of other HST galaxies for which Cepheid distances have been derived (e.g., Ferrarese et al. 2000; see also Tanvir 1999). An approved Cycle 10 HST program to derive a Cepheid-based distance to NGC 1637 is currently underway, and the results will be directly compared with the EPM distances derived to SN 1999em.

\section{Conclusions}

We present 30 optical spectra and 49 photometric epochs of SN 1999em sampling the first 517 days of its development. SN 1999em displays the major photometric and spectral characteristics of a SN II-P, exhibiting a distinct photometric plateau lasting about 100 days from explosion, and well-developed P-Cygni spectral line features. We suspect that SN 1999em suffers from minimal reddening, and concur with the range of possible values found by Baron et al. (2000), and adopt $E(B-V)=0.10 \pm 0.05$ mag.

We find a number of interesting features in the spectral evolution of the P-Cygni line profiles, particularly $\mathrm{H} \alpha$. We identify the dominant ions responsible for most of the absorption features seen in the optical portion of the spectrum of SN 1999em during the plateau phase. The photospheric velocity derived from four weak, unblended absorption features is found to be $5 \%-10 \%$ lower than that derived using the stronger Fe II $\lambda \lambda 4924,5018,5169$ lines, which have traditionally been used to estimate the photospheric velocity in EPM studies. The velocity offset between the weakest lines and the stronger Fe II features is shown to exist in other SNe II as well. If this is a generic feature of SNe II and weaker lines are indeed a better tracer of photospheric velocity, then EPM estimates previously made with the stronger Fe II $\lambda \lambda 4924,5018,5169$ lines are skewed by $5 \%-10 \%$ toward larger distances. We note that the comparison of EPM distances with the most

recent Cepheid distances yields $D_{\text {Cepheids }} / D_{\mathrm{EPM}}=0.87 \pm 0.06$, a discrepancy that is in the same sense (i.e., $D_{\text {EPM }}>D_{\text {Cepheids }}$ ) as the aforementioned velocity offset; difficulties in this comparison exist, however, due to the peculiar nature of several of the SNe II, the use of Cepheid distances to 
galaxies that are only in the same group in which the SN occurred (i.e., not the host galaxy itself), and existing discrepancies in the Cepheid distance scale.

We derive an EPM distance to SN 1999em using the flux dilution factors $(\zeta)$ of E96 as modified by Hamuy et al. (2001). We use three different filter combinations ( $B V, B V I$, and $V I)$ to determine the photospheric color temperature. The total spread in the distances derived using the different filter combinations is about $15 \%$ (when using the weak, unblended absorption features to estimate photospheric velocity), in the sense $D_{V I}>D_{B V I}>D_{B V}$. These discrepancies cannot be explained by statistical uncertainty in the measured quantities, and must be systematic in nature, most likely due to uncertainty in the dilution factor and perhaps aggravated by the lack of models used to derive the dilution factor with properties similar to SN 1999em: SN 1999em became redder more quickly than the prototypical Type II-P SN 1969L, and reached color temperatures lower than any model studied by E96 about half way through the plateau phase. Taking the observed scatter among the different filter combinations into account, our best estimate for the distance to SN $1999 \mathrm{em}$ is $D=8.2_{-0.6}^{+0.6} \mathrm{Mpc}$, with an explosion date of $t_{\circ}=$ HJD $2,451,475.6_{-1.4}^{+1.4}$, or $5.3 \pm 1.4$ days before discovery. This leads to an extinction-corrected peak $B$-band magnitude of $M_{B}=-16.2 \pm 0.2 \mathrm{mag}$, and an average $V$-band plateau brightness of $\bar{M}_{V}$ (plateau) $=-15.9 \pm 0.2 \mathrm{mag}$, which is somewhat fainter than average SNe II-P.

Finally, we investigate the theoretical prediction by Höflich et al. (2000) that SNe II-P should exhibit a rather small dispersion in their average $V$-band magnitude during the plateau phase. Their unique light-curve shape (making spectral identification unnecessary) and certain existence at high redshift make them attractive cosmological tools. From 8 SNe II-P with previously published distances and SN $1999 \mathrm{em}$, we find $\bar{M}_{V}$ (plateau) $=-16.4_{-0.6}^{+0.6}$. If this small sample is representative of all SNe II-P, then distances to SNe II-P accurate to $\sim 30 \%(1 \sigma)$ may be possible without having to acquire the extensive data set required for an EPM analysis, thereby increasing the viability of this class of objects as cosmological beacons at $z>2$.

We thank Wil J. M. van Breugel, Daniel Stern, David R. Ardila, Robert H. Becker, Michael S. Brotherton, James W. Colbert, Willem H. de Vries, Richard Edelson, George K. Miley, Michiel Reuland, Adam G. Riess, S. Adam Stanford, and Richard L. White for assistance with the observations, and Shashi M. Kanbur, Jeffery A. Newman, and Nial A. Tanvir for helpful discussions. We thank the referee, Brian Schmidt, for very helpful comments and suggestions that resulted in an improved manuscript. Some of the data presented herein were obtained at the W. M. Keck Observatory, which is operated as a scientific partnership among the California Institute of Technology, the University of California, and the National Aeronautics and Space Administration. The Observatory was made possible by the generous financial support of the W. M. Keck Foundation. We are grateful to the Keck staff for their support of the telescopes. This research has made use of the NASA/IPAC Extragalactic Database (NED), which is operated by the Jet Propulsion Laboratory, California Institute of Technology, under contract with NASA. We have made use of the LEDA database (http://leda.univ-lyon1.fr). Our work was funded by NASA grants GO- 
7821, GO-8243, and GO-8648 from the Space Telescope Science Institute, which is operated by AURA, Inc., under NASA contract NAS 5-26555. Additional funding was provided to A. V. F. by NASA/Chandra grant GO-0-1009C, NSF grants AST-9417213 and AST-9987438, and by the Guggenheim Foundation. KAIT was made possible by generous donations from Sun Microsystems, Inc., the Hewlett-Packard Company, AutoScope Corporation, the National Science Foundation, the University of California, Lick Observatory, and the Sylvia and Jim Katzman Foundation.

\section{REFERENCES}

Arnett, W. D., Bahcall, J. N., Kirshner, R. P., \& Woosley, S. E. 1989, ARA\&A, 27, 629

Baade, W. 1926, Astr. Nachr., 228, 359

Barbon, R., Benetti, S., Cappellaro, E., Rosino, L., \& Turatto, M. 1990, A\&A, 237, 79

Baron, E., et al. 1995, ApJ, 441, 170

Baron, E., et al. 2000, ApJ, 545, 444

Bessell, M. S. 1990, PASP, 102, 1181

Bessell, M. S. 1999, PASP, 111, 1426

Bloom, J. S., et al. 1999, Nature, 401, 453

Branch, D. 1987, ApJ, 320, L121

Burstein, D., \& Heiles, C. 1982, AJ, 87, 1165

Chevalier, R. A., \& Soker, N. 1989, ApJ, 341, 867

Chugai, N. N. 1991, Soviet Astr. Lett., 17, 400

Clocchiatti, A., Wheeler, J. C., Barker, E. S., Filippenko, A. V., Matheson, T., \& Liebert, J. W. 1995, ApJ, 446, 167

Cohen, M. H. 1996, The LRIS Polarimeter (Keck Observatory instrument manual), available at http://www2.keck.hawaii.edu:3636/

Coil, A. L., et al. 2000, ApJ, 544, L111

Cordes, J. M., \& Chernoff, D. F. 1998, ApJ, 505, 315

Cousins, A. W. J. 1981, South African Astron. Obs. Circ., 6, 4 
Da Costa, G. S. 1992, in Astronomical CCD Observing and Reduction Techniques, ed. S. B. Howell (San Francisco: ASP), 90

Eastman, R. G., \& Kirshner, R. P. 1989, ApJ, 347, 771

Eastman, R. G., \& Pinto, P. A. 1993, ApJ, 412, 731

Eastman, R. G., Schmidt, B. P., \& Kirshner, R. 1996, ApJ, 466, 911 (E96)

Eastman, R. G., Woosley, S. E., Weaver, T. A., \& Pinto, P. A. 1994, ApJ, 430, 300

Elias, J. H., et al. 1988, ApJ, 331, L13

Fassia, A., et al. 1998, MNRAS, 299, 150

Ferrarese, L., et al. 2000, ApJS, 128431

Filippenko, A. V. 1982, PASP, 94, 715

Filippenko, A. V. 1997, ARA\&A, 35, 309

Filippenko, A. V., Porter, A. C., Sargent, W. L. W., \& Schneider, D. P. 1986, AJ, 92, 1341

Filippenko, A. V., \& Sargent, W. L. W. 1986, AJ, 91, 691

Freedman, W. L., et al. 2001, ApJ, 553, 47

Hamuy, M., et al. 2001, ApJ, in press (astro-ph/0105006)

Hamuy, M., et al. 1994, AJ, 108, 2226

Hamuy, M., Suntzeff, M. B., Gonzales, R., \& Martin, G. 1988, AJ, 95, 63

Hatano, K., Branch, D., Fisher, A., Millard, J., \& Baron, E. 1999, ApJS, 121, 233

Höflich, P. 1991, A\&A, 246, 481

Höflich, P. 1995, ApJ, 440, 821

Höflich, P., Khokhlov, A., \& Wang, L. 2001, to appear in the 20th Texas Conference on Relativistic Astrophysics ed. H. Martel (New York: AIP) (astro-ph/0104025)

Höflich, P., Straniero, O., Limongi, M., Dominguez, I., \& Chieffi, A. 2001, to appear in The Seventh Texas-Mexico Conference on Astrophysics at Austin ed. W. Lee \& S. Torres-Peimbert (astroph/0005037)

Höflich, P., Wheeler, J. C., \& Wang, L. 1999, ApJ, 521, 179

Horne, K. 1986, PASP, 98, 609 
Issa, M. R., MacLaren, I., \& Wolfendale, A. W. 1990, A\&A, 236, 237

Jeffery, D., \& Branch, D. 1990, in Supernovae, eds. J. C. Wheeler, T. Piran, \& S. Weinberg (Singapore: World Scientific), 149

Jha, S., Challis, P., Garnavich, P., Kirshner, R., Calkins, M., \& Stanek, K. 1999, IAU Circ. No. 7296

Johnson, H. L., Mitchell, R. I., Iriarte, B., \& Wisniewski, W. Z. 1966, Commun. Lunar Plate. Lab., 4,99

Kirshner, R. P., \& Kwan, J. 1974, ApJ, 193, 27

Kurucz, R. L. 1996, Atomic Spectral Line Database, http://cfa-www.harvard.edu/amdata/ampdata/kurucz23/sekur.html

Landolt, A. U. 1992, AJ, 104, 340

Leonard, D. C., Filippenko, A. V., Ardila, D. R., \& Brotherton, M. S. 2001, ApJ, 553, 861 (L01)

Leonard, D. C., \& Filippenko, A. V. 2001, PASP, 113, 920

Li, W. D. 1999, IAU Circ. No. 7294

Li, W. D., et al. 2000, in Cosmic Explosions, ed. S. S. Holt \& W. W. Zhang (New York: AIP), p. 103

Massey, P., \& Gronwall, C. 1990, ApJ, 358, 344

Matheson, T., Filippenko, A. V., Ho, L. C., Barth, A. J., \& Leonard, D. C. 2000, AJ, 120, 1499

Matzner, C. D., \& McKee, C. F. 1999, ApJ, 510, 379

McGregor, P. 1988, Proc. Astron. Soc. Aust., 7, 450

Meikle, W. P. S., Spyromilio, J., Allen, D. A., Varani, G.-F., \& Cumming, R. J. 1993, MNRAS, 261,535

Miller, D. L., \& Branch, D. 1990, AJ, 100, 530

Miller, J. S., \& Stone, R. P. S. 1993, Lick Obs. Tech. Rep., No. 66

Modjaz, M., Li, W., Filippenko, A. V., King, J. Y., Leonard, D. C., Matheson, T., \& Treffers, R. R. 2001, PASP, 113, 308

Montes, M., \& Wagoner, R. V. 1995, ApJ, 445, 828

Munari, U., \& Zwitter, T. 1997, A\&A, 318, 269 
Oke, J. B., \& Gunn J. E. 1983, ApJ, 266, 713

Oke, J. B., et al. 1995, PASP, 107, 375

Parodi, B. R., Saha, A., Sandage, A., \& Tammann, G. A. 2000, ApJ, 540, 634

Patat, F., Barbon, R., Cappellaro, R., \& Turatto, M. 1994, A\&A, 282, 731

Phillips, A. P., \& Heathcote, S. R. 1989, PASP, 101, 137

Phillips, M. M., Heathcote, S. R., Hamuy, M., \& Navarrete, M. 1988, AJ, 95, 1087

Pooley, D., et al., 2001, MNRAS, submitted (astro-ph/0103196)

Press, W. H., Teukolsky, S. A., Vetterling, W. T., \& Flannery, B. P. 1992, Numerical Recipes in C: The Art of Scientific Computing, 2nd edition, (Cambridge: Cambridge University Press)

Richmond, M. W., et al. 1995, AJ, 109, 2121

Saha, A., et al. 1999, ApJ, 522, 802

Savage, B. D., \& Mathis, J. S. 1979, ARA\&A, 17, 73

Schlegel, D. J., Finkbeiner, D. P., \& Davis, M. 1998, ApJ, 500, 525

Schmidt, B. P., Kirshner, R. P., \& Eastman, R. G. 1992, ApJ, 395, 366

Schmidt, B. P., et al. 1994a, ApJ, 432, 42

Schmidt, B. P., et al. 1993, AJ, 105, 2236

Schmidt, B. P., et al. 1994b, AJ, 107, 1444

Schmutz, W., Abbott, D. C., Russell, R. S., Hamann, W. R., \& Wessolowski, U. 1990, ApJ, 355, 255

Silbermann, N. A., et al. 1999, ApJS, 515, 1

Sohn, Y.-J., \& Davidge, T. J. 1998, AJ, 115, 130

Stetson, P. B. 1987, PASP, 99, 191

Stetson, P. B. 1991, in 3rd ESO/ST-ECF Data Analysis Workshop, ed. P. J. Grosbol \& R. H. Warmels, ESO Conf. and Workshop Proc. No. 38 (ESO: Garching bei Munchen), 187

Stone, R. P. S. 1977, ApJ, 218, 767

Stone, R. P. S., \& Shields, J. 1990, Lick Obs. Tech. Rep., No. 52

Suntzeff, N. B. 2000, in Cosmic Explosions, ed. S. S. Holt \& W. W. Zhang (New York: AIP), 65 
Suntzeff, N. B., et al. 1999, ApJ, 117, 1175

Tanvir, N. R. 1999, in Harmonising Distance Scales in a Post Hipparcos Era, ed. D. Egret \& A. Heck (San Francisco: ASP), 84

Tanvir, N. R., Ferguson, H. C., \& Shanks, T. 1999, MNRAS, 310, 175

Treffers, R. R., Peng, C. Y., Filippenko, A. V., Richmond, M. W., Barth, A. J., \& Gilbert, A. M. 1997, IAU Circ. No. 6627

Turatto, M., Cappellaro, E., Benetti, S., \& Danziger, I. J. 1993, MNRAS, 265, 471

Udalski, A., et al. 1999, Acta. Astr., 49, 201

Utrobin, V. P., Chugai, N. N., \& Andronova, A. A. 1995, A\&A, 295, 129

van Zee, L., et al. 1998, AJ, 116, 2805

Wade, R. A., \& Horne, K. 1988, ApJ, 324, 411

Wagoner, R. V. 1982, in Supernovae: A Survey of Current Research, ed. M. J. Rees \& R. J. Stoneham (Dordrecht: Reidel), 253

Wagoner, R. V. 1991, in Supernovae, ed. S. E. Woosley (New York: Springer-Verlag), 741 (W91)

Wang, L., Howell, D. A., Höflich, P., \& Wheeler, J. C. 2001, ApJ, 550, 1030

Wang, L., \& Wheeler, J. C. 1996, ApJ, 462, L27

Weaver, T. A., \& Woosley, S. E. 1993, Phys. Rep. 227, 65

Wheeler, J. C. 2000, in Cosmic Explosions, ed. S. S. Holt \& W. W. Zhang (New York: AIP), 445

Williams, R. E. 1987, ApJ, 320, L117

This preprint was prepared with the AAS LATEX macros v5.0. 